\title{
Stimulus-active polymer actuators for next-generation microfluidic devices
}

\author{
Wolfgang Hilber ${ }^{1}$ (D)
}

Received: 16 March 2016/Accepted: 5 July 2016/Published online: 20 July 2016

(C) The Author(s) 2016. This article is published with open access at Springerlink.com

\begin{abstract}
Microfluidic devices have not yet evolved into commercial off-the-shelf products. Although highly integrated microfluidic structures, also known as lab-on-a-chip (LOC) and micrototal-analysis-system ( $\mu$ TAS) devices, have consistently been predicted to revolutionize biomedical assays and chemical synthesis, they have not entered the market as expected. Studies have identified a lack of standardization and integration as the main obstacles to commercial breakthrough. Soft microfluidics, the utilization of a broad spectrum of soft materials (i.e., polymers) for realization of microfluidic components, will make a significant contribution to the proclaimed growth of the LOC market. Recent advances in polymer science developing novel stimulus-active soft-matter materials may further increase the popularity and spreading of soft microfluidics. Stimulus-active polymers and composite materials change shape or exert mechanical force on surrounding fluids in response to electric, magnetic, light, thermal, or water/solvent stimuli. Specifically devised actuators based on these materials may have the potential to facilitate integration significantly and hence increase the operational advantage for the end-user while retaining costeffectiveness and ease of fabrication. This review gives an overview of available actuation concepts that are based on functional polymers and points out promising concepts and trends that may have the potential to promote the commercial success of microfluidics.
\end{abstract}

Wolfgang Hilber

Wolfgang.Hilber@jku.at

1 Institute for Microelectronics and Microsensors, Johannes Kepler University Linz, Linz, Austria

\section{Introduction}

The field of microfluidics comprises research into and development of miniaturized systems for the handling, treatment, metering, and analysis of small amounts of liquids or gases [1]. The corresponding lab-on-a-chip (LOC) and micrototal-analysis-system ( $\mu$ TAS) devices [2] have repeatedly been predicted to revolutionize fluid analysis due to their potential to replace bulky and costintensive bench-top equipment and the associated manual handling of large amounts of biological and chemical reagents. However, despite significant research activities in this highly multidisciplinary field over more than two decades, only few concepts have ultimately reached the level of commercialization. Studies addressing this issue $[3,4]$ identified the lack of standardization and integration as the main barriers to acceptance by the end-user and thus to commercial breakthrough: After acquiring one of the few commercially available microfluidic products, the operator may face difficulties in connecting the microfluidic device to ancillary hardware, such as external supplies, valves, pumps and other microfluidic components [5]. In contrast to assessments in the early stages [6], which predicted silicon-based microsystem technology as the most promising approach for microfluidic applications, soft-matter-based and hybrid solutions have become more significant [7]. However, polydimethylsiloxane (PDMS), the most popular polymer for realizing microfluidic components at the laboratory scale, is usually avoided by the manufacturing sector mainly due to difficulties with processing and technology at the industrial scale. Compared to other commercially utilized standard polymers such as polycarbonate (PC) and poly(methyl methacrylate) (PMMA), PDMS is a relatively expensive material whose large-scale 
production requires enormous effort if the level of quality and reliability of the final products expected by the potential end-user is to be reached. Recent developments in the field of functional polymers-that is, softmatter materials which respond to an external, mainly physical, stimulus - may be essential in contributing to the foreseen commercial success of microfluidic concepts. Most of these functional polymers do not respond to a single stimulus, but rather to a set of physical stimuli. This review intends to introduce a clear structure to the class of functional polymers used for microfluidic actuation and to bridge a gap in the scientific literature. First, a generic classification of microfluidic actuation concepts, the basic deformation modes of the functional materials, and the material classes according to physical stimuli are introduced (Sect. 2). In the following sections, a variety of functional materials are presented, starting with concepts based on PDMS, which is in pure state barely responsive to external stimuli, but becomes responsive when combined with other materials in the form of layered structures or composites (Sect. 3). Next, the multifaceted material class of polymer hydrogels (PHs) is discussed; this class of polymers can be made responsive to almost any conceivable physical stimulus, ranging from temperature to $\mathrm{pH}$ value, light, water and solvent concentration, electric and magnetic fields, and chemical reactions that cause self-oscillating actuation of the PH (Sect. 4). Electroactive polymers (EAPs), which can be divided into electronic and ionic EAPs, are discussed in the subsequent sections (Sects. 5, 6). Generally speaking, electronic EAPs respond to electrostatic charges. This group includes electrostrictive ferroelectric and relaxor ferroelectric polymers such as polyvinylidene fluoride (PVDF) and related co- and ter-polymers, partlyliquid crystal elastomers (LCEs), and dielectric elastomer actuators (DEAs). The section about PDMS-based actuation schemes also covers DEAs, since most of the presented DEAs use PDMS as their elastomeric component. The actuation mechanism in ionic EAPs, in contrast, is based on diffusion or-more generally-on the mobility of ions in the functional material (Sects. 7, 8). For example, ionic polymer-metal composites and conductive (i.e., conjugated) polymers such as polypyrrole (PPy) and polyaniline (PANI) belong to this group. The final sections of this paper describe phase-change actuator (PCA) materials (Sect. 9), shape-memory polymers (SMPs) (Sect. 10), and electro- (ERFs) and magnetorheological fluids (MRFs) (Sect. 11). Conclusion (Sect. 12) gives an overview of the different polymerbased actuation concepts, assesses their advantages and disadvantages when used in microfluidics, and seeks to identify promising concepts and technologies for the future.

\section{From components to materials}

\subsection{Basic elements of microfluidic LOC and $\mu$ TAS devices}

Besides the fluid channels, which typically feature dimensions in the range between $1 \mu \mathrm{m}$ and $0.5 \mathrm{~mm}$, advanced microfluidic chips should be equipped with active elements for pumping, mixing, and valving. The fluid volume to be handled strongly depends on the actual application. Manipulation of single cells may require fluid volumes in the order of picoliters only, whereas in the area of drug discovery and DNA amplification sample volumes may range from parts of a nanoliter to parts of a milliliter. Active cooling of microelectronic components even requires the handling of tens of milliliters per minute. Likewise the specified lifetime ranges from only a few minutes for single use or disposable elements, to days or weeks for reusable biochemical assay chips, to thousands of operating hours for electronic cooling devices. At the current state pumping is in the main done offchip, either with connected syringe pumps or other pressure sources, or by utilizing capillary forces in the low volume regime. For valving the situation is quite similar, here pneumatic actuation of thin membranes from the outside is the overwhelmingly used method to block or release a fluid channel. Figure 1 shows a typical example in the field of molecular diagnostics [8].

On chip the most popular approach for fluid actuation is the use of flexible diaphragms, which, for example when driven by piezoelectric or magnetic forces, can execute valve functionalities, or, driven in a reciprocating manner and combined with check valves or purely passive nozzlediffuser valves, can operate as micropumps. These socalled positive displacement pumps typically achieve flow

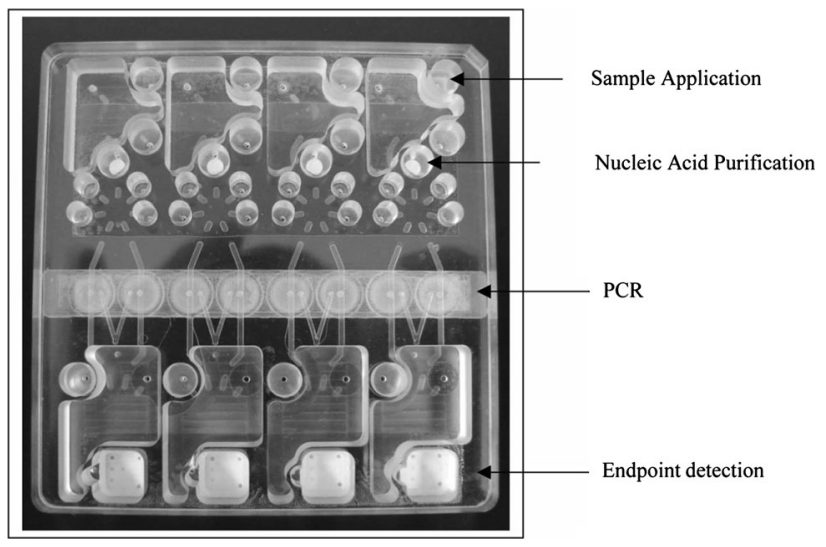

Fig. 1 Rheonix $\mathrm{CARD}^{\circledR}$ capable of performing up to four simultaneous molecular assays. The chip contains valve/pump assemblies based on deformable membranes operated with a pressure source offchip. Reproduced with permission from Rheonix, Inc. 
Fig. 2 Insulin micropump assembly. (Top) Schematic cross section of the siliconbased micropump with integrated valves consisting of a stack of three plates (Si-SOI-Si). a Micropump mounted onto a ceramic substrate having interconnection pads. b Disposable unit with the pump and the battery mounted onto the insulin reservoir. From [11], (C)2014 MDPI. Reproduced with permission
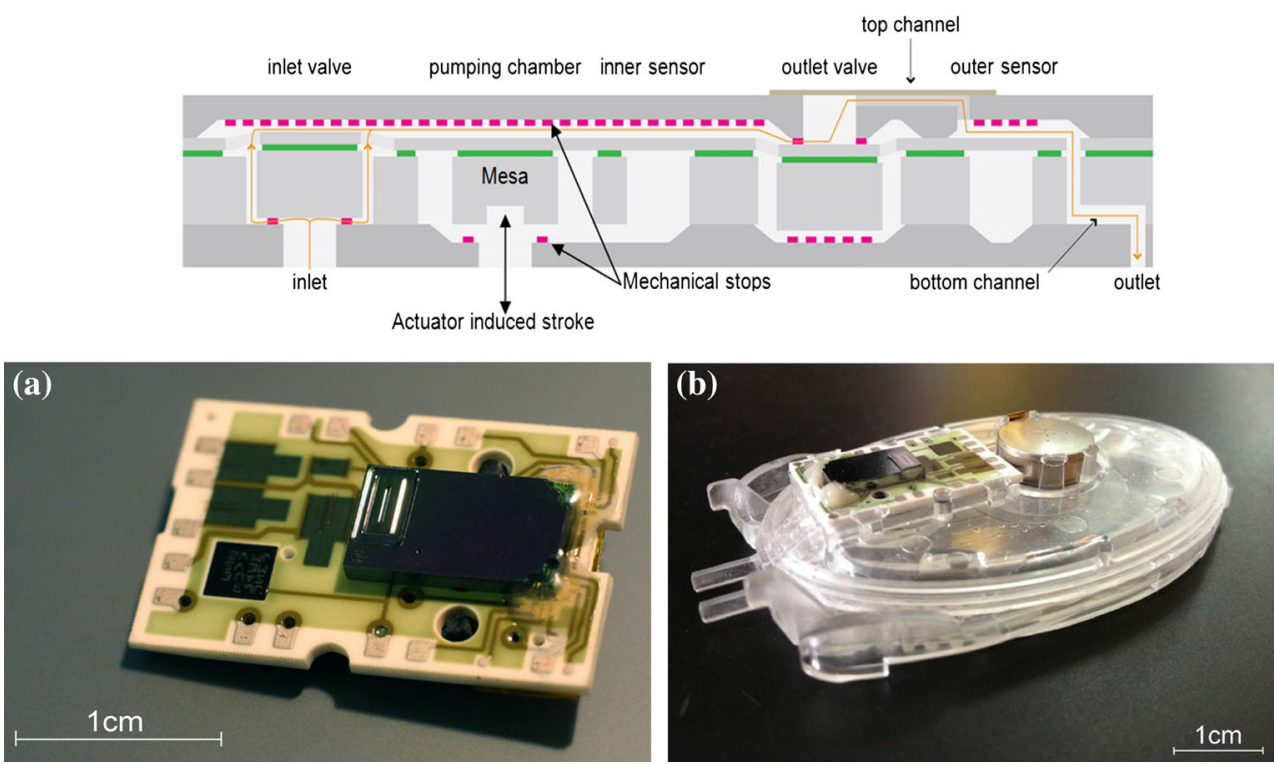

rates in the range between $10 \mu \mathrm{l} / \mathrm{min}$ and $10 \mathrm{ml} / \mathrm{min}$ with a maximum pressure from 1 to $100 \mathrm{kPa}$ [9]. The corresponding valves achieve maximum switching pressures in the range between 20 and $200 \mathrm{kPa}$ with switching times between $1 \mathrm{~ms}$ and $10 \mathrm{~s} \mathrm{[10].} \mathrm{Figure} 2$ shows, as an example for an integrated LOC device, an insulin micropump realized in silicon technology [11].

The technological effort for realization of such an integrated device is relatively high: It requires siliconbased MEMS technology, hybrid joining technology with metallic interconnects on a separate ceramic substrate and final assembly steps with a polymer case carrying the liquid. Stimulus-responsive polymers are expected to reduce the technological effort as well as the manufacturing costs of highly integrated microfluidic devices considerably. The focus of this review is (1) to show up the broad range of materials that can be used to realize soft actuators, (2) to screen the concepts that have been realized so far, and (3) to point up the strengths and weaknesses for design and development of fully integrated, self-contained microfluidic chips.

\subsection{Generic microfluidic components}

Microfluidic actuation using stimulus-responsive polymers can be downscaled and classified according to a basic set of generic actuation principles, as shown in Fig. 3. First, the actuation principles can be distinguished with respect to the two main applications in the microfluidic field: pumping/ mixing (a)-(d) and valving (e)-(f). By far the most popular approach to fluid actuation in microfluidic structures uses flexible/movable membranes that either produce a continuous piston displacement (a) or, in combination with valve

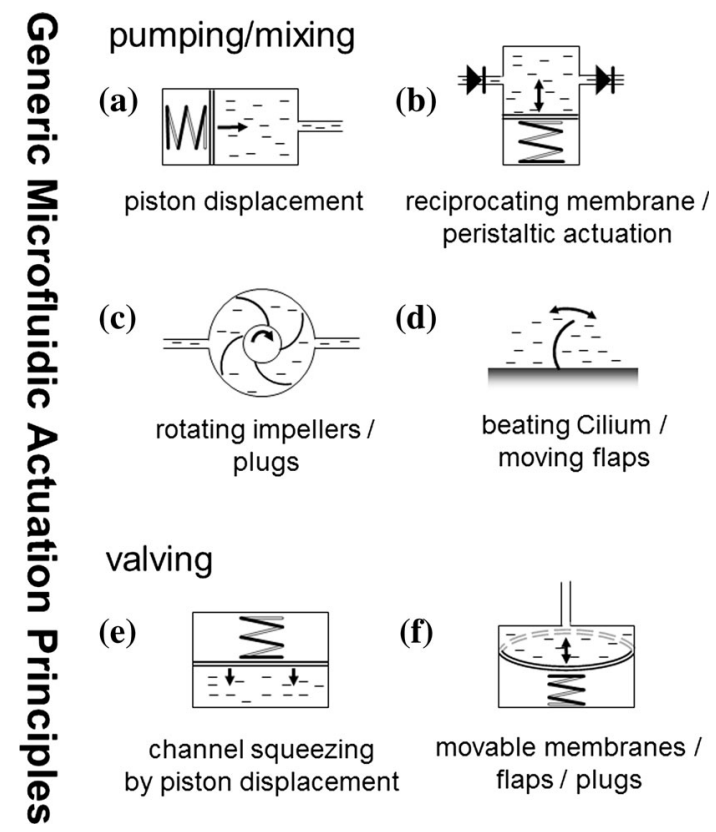

Fig. 3 Classification of microfluidic actuation concepts that are based on stimulus-responsive polymers

structures at the inlet and outlet, apply reciprocating pressure strokes to a confined fluid volume (b). The prevalence of these concepts arises on the one hand from their ability to move substantial fluid volumes even against high back pressure and on the other hand from the simple structure which requires only moderate technological effort. Aside from membrane pumps, which have been scaled down successfully for use in microfluidics, some rotary pump concepts have been demonstrated (c). However, they are far less common, most probably due to technological 
difficulties with integrating tiny and freely rotating structures into polymeric devices. Biomimetic approachespredominantly mimicking the beating behavior of cilia and flagella (i.e., flexible extensions of the biological cell membrane primarily for locomotion of single cells or microorganisms) - have successfully been fabricated with a set of polymeric materials and have proved to be particularly suitable for mixing applications and low-volume fluid transport (d). The ciliary and flagellar beating pattern is characterized by a series of bends and twists starting at the base of the cellular extension and propagating to its end. Valving applications, in contrast, are usually based on mechanisms for controlled vertical or lateral squeezing of the fluid channel (e). A few concepts use deformable membranes or flaps for blocking and releasing a channel entry, sometimes combined with bistable structures in order to improve response time and/or actuation amplitude (f).

\subsection{Basic actuator deformation modes}

On closer examination of these microfluidic actuation concepts, one can identify a set of basic deformation modes of the stimulus-responsive material. The simplest classification is based on the dimensionality of the structural change of the material in response to an external stimulus, as illustrated in Fig. 4: The three basic deformation modes are one-dimensional bending of cantilever- or beam-like structures, two-dimensional membrane deformations, and three-dimensional volume expansions. Two-dimensional

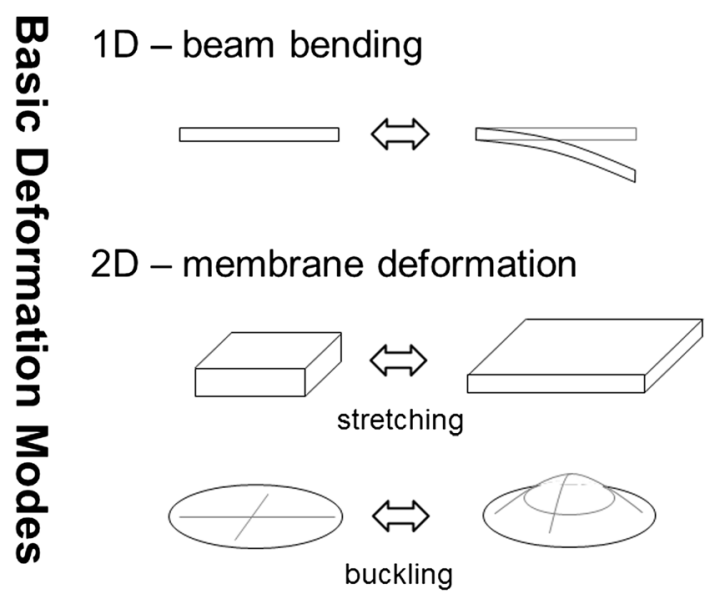

3D - volume expansion

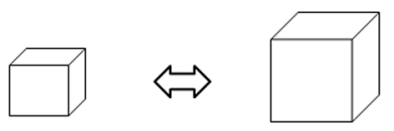

Fig. 4 Basic deformation modes of stimulus-responsive polymers membrane deformations can be further divided into lateral stretching modes, which are accompanied by thinning of the membrane, and vertical displacement modes, which are characterized by non-uniform buckling of the membrane, with the greatest displacement typically in the center.

Virtually any kind of stimulus-responsive material can be used to create one of the deformation modes described above, although certain materials may be preferable to realize a specific type of deformation. For example, the swelling behavior of polymer hydrogels or phase-change materials is convenient when volume expansion is required, and the squeezing shape transformation of conjugated and ferroelectric polymers may be ideally suited to achieving various kinds of membrane deformations. Figure 5 illustrates the basic physical changes under stimulation for the most prominent classes of stimulus-responsive polymers. These are (1) change in mechanical properties, (2) non-uniform strain generation, and (3) change in volume.

However, aside from suitability of a specific material for the desired deformation mode, the major selection criterion is the choice of stimulus.

\subsection{Materials and stimuli}

Figure 6 relates the most popular material classes for realizing responsive polymer actuators in microfluidics to possible, mainly physical, stimuli. From Fig. 6 it is evident that some stimulation mechanisms are preferred for microfluidic actuation, in particular electric and magnetic fields, temperature and-somewhat related-radiation/light (almost respectively entirely filled segments in Fig. 6). Most probably these stimuli are preferred over biological, chemical, pH value, and water-/solvent-based mechanisms because of issues with integrability and controllability at small scales. Further, some polymers that are naturally responsive primarily to one stimulus have successfully been made responsive to a whole set of stimuli by (1) modification of the material itself, (2) fabrication of composites with suspended particles that introduce an additional functionality, or (3) combination with other materials in stacked or layered geometries. Polymer hydrogels, for example, have been made responsive to every conceivable stimulus, thus appearing in every segment of Fig. 6. In simple terms, the actuation mechanism of polymer hydrogels is based on the amount of water stored in the polymer matrix, and the actuation concepts basically aim to control the water content in various ways. PDMS-based actuators are unique in that the base material is unresponsive in its pure form. This biocompatible and transparent elastomer is made responsive by admixing functional particles prior to the cross-linking process or by depositing metal and/or functional layers on its surface. 

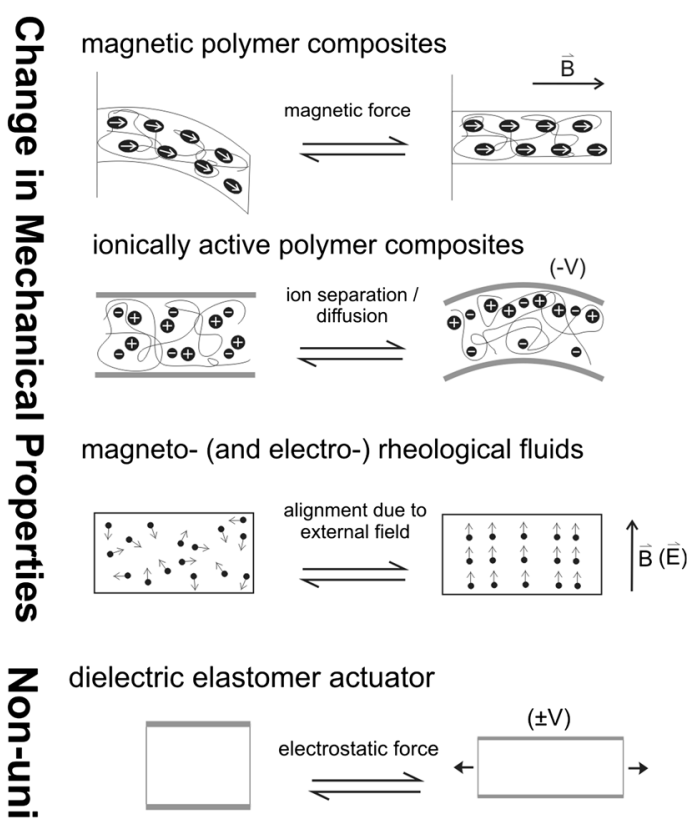

electrostrictive ferroelectric polymers

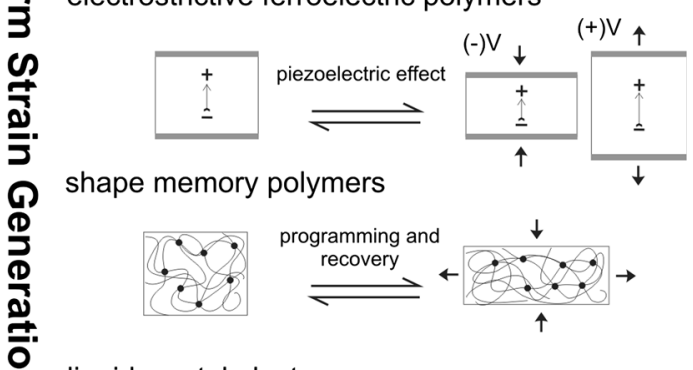

liquid crystal elastomers
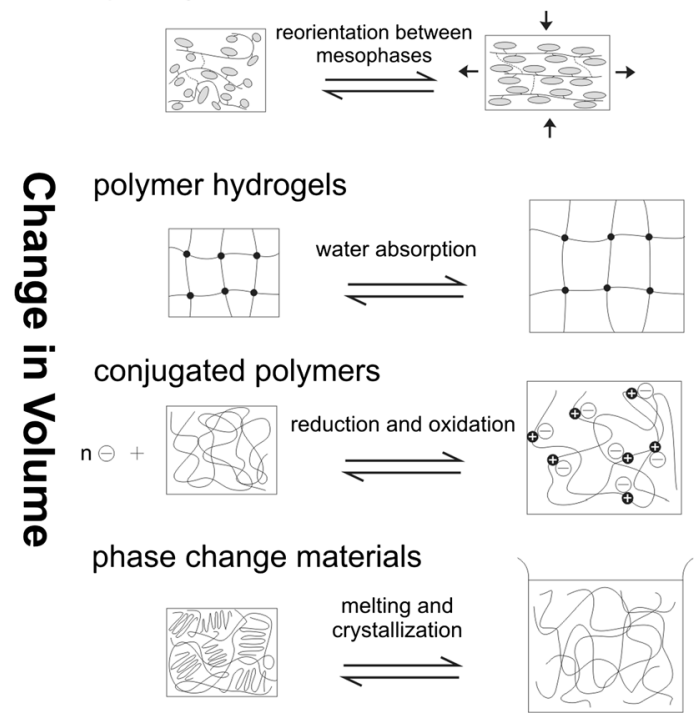

Fig. 5 Physical changes under stimulation

The practice works best to achieve responsiveness to any type of electromagnetic signal, compare Fig. 6. Other types of responsiveness are inherently related to the intrinsic properties of the material, such as the water/solvent and $\mathrm{pH}$ value sensitivity of shape-memory polymers and the sensitivity of electroactive conjugated polymers to humidity, and can be considered as a disadvantageous kind of crosssensitivity. Considered as an advantage, however, the concurrent sensitivity of specific functional polymers to more than one stimulus facilitates the implementation of complex actuation schemes. Blank areas in Fig. 6 illustrate that a material is insensitive to a particular stimulus, which is important for two reasons: First, this information is crucial to the design of microfluidic components in order to avoid spurious and interference effects. Second, the blank areas represent potential fields of activity for fundamental and material-related research. Progress in developing novel stimulus-active materials will not only fill the gaps in this scheme and increase the range of materials available for realizing polymer-based transducers, but it will eventually also increase use and popularity of microfluidic components. A good example to this effect is the evolution of PDMS from a polymer for pure casting of microfluidic channels to a multifunctional material that has been used for realization of manifold microfluidic transducers.

\section{PDMS-based actuators}

Since the pioneering work of G.M. Whitesides et al. [12-14] in the late nineties, describing procedures for rapid prototyping of microfluidic structures, PDMS has become the best-known and most popular material for realizing microfluidic structures in scientific research and deservedly so. It is optically transparent, mechanically strong but flexible, chemically inert, biocompatible, and highly gas permeable, has excellent working properties, can be processed in any laboratory without the need for expensive equipment, and can be combined with a large variety of other materials. Schneider et al. [15] explored the mechanical properties, creep behavior, and thermal aging of the most commonly used PDMS products in soft lithography, namely RTV 615 from Bayer Silicones and Sylgard 184 from Dow Corning. It has been demonstrated that addition of the UV-sensitive photoinitiator benzophenone leads to a photodefinable PDMS material [16]. Thus, by using standard UV-photolithography, multilayer structures, and multilevel microfluidic chips based on PDMS can be fabricated. Further, it has been shown that the same technological approach can be used to realize silicone rubber films with graded and locally modified mechanical properties [17]. PDMS bilayer films with embedded spontaneous curvatures have been realized by preparing PDMS/(PDMS + silicone oil) cross-linked bilayers and extracting the oil with a suitable organic solvent [18]. The most common configuration of PDMS-based actuators is a thin membrane which, actuated by external power sources, 
Fig. 6 Materials classified according to type of stimulus

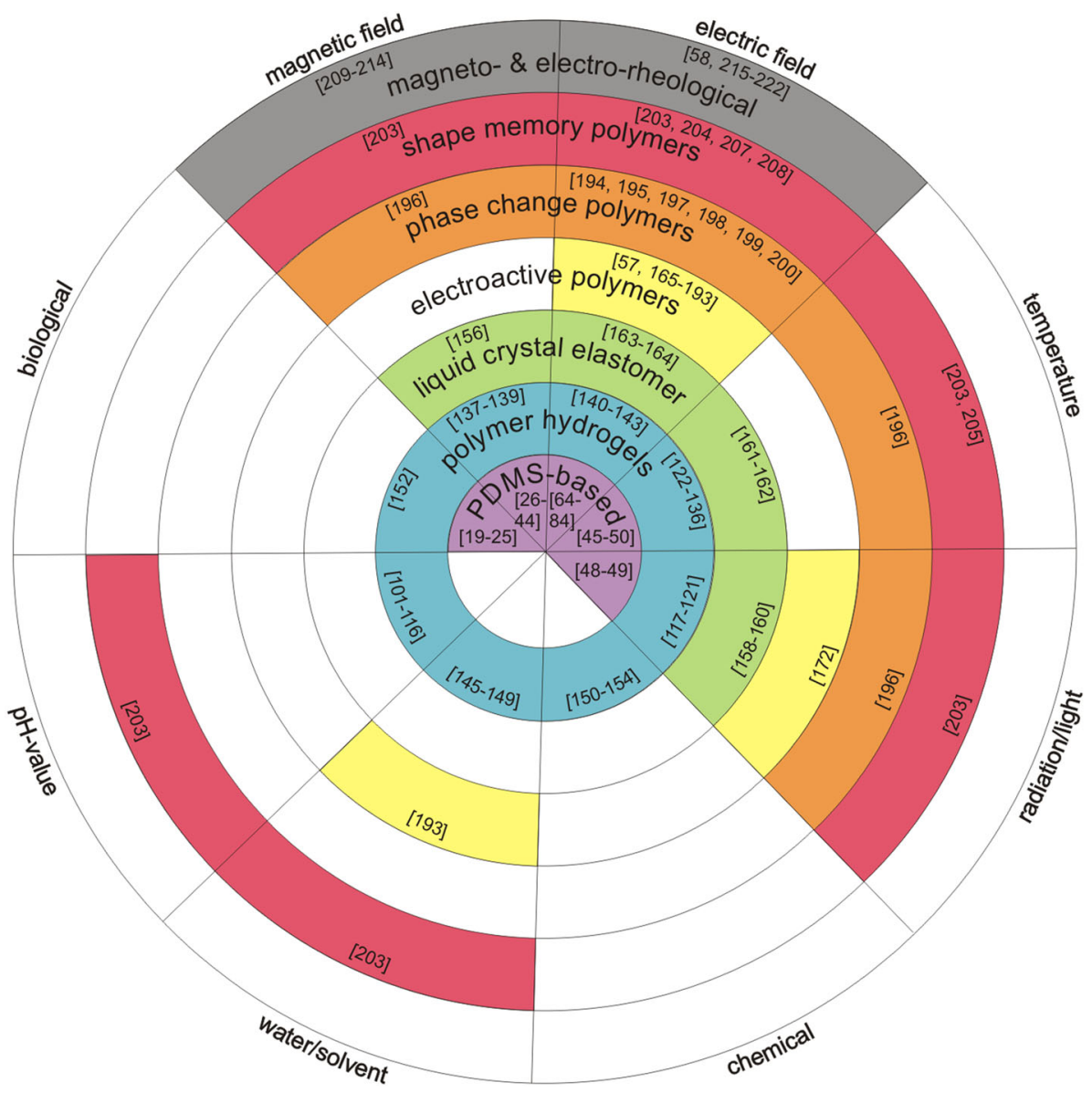

induces pressure strokes in a confined fluid volume or blocks/releases a channel entry. The actuation mechanisms range from thermopneumatic to electromagnetic, electrostatic, and electrochemical principles. This review does not include actuator approaches based on pure PDMS, except for extraordinarily innovative concepts. Instead, the focus is on modified PDMS in composites, in layered structures or with specifically designed geometries that allow the realization of specifically devised actuator schemes for microfluidic applications. An innovative concept to begin with is the combination of living contractive cells with soft polymer structures in order to achieve autonomous fluid actuation.

\subsection{Bionic approach—cardiomyocytes}

In 2007, Tanaka et al. [19] reviewed the first applications of biological cells on microchips. The concepts ranged from biomicroactuators driven by a single or a few cells or microorganisms for actuating microobjects to concepts based on numerous cells or tissue for actuating larger objects and fluids. Cardiomyocytes, the muscle cells which make up the cardiac muscle, were identified as promising candidates for the realization of cell-based actuators. Unlike conventional microactuators, these spontaneously contracting cells are autonomously powered by glucose as an energy source and operate without external stimuli. Typically, sheets of cardiomyocytes on PDMS membranes are used for fluid actuation. Pilarek et al. [20] reviewed the application of biological cardiomicropumps in microbioreactors and analytical microsystems. Until recently cardiocell-based actuators required the harvesting of primary neonatal cardiomyocytes, which is both practically and ethically difficult. However, a method has now been developed that uses induced pluripotent stem (iPS) cells obtained from subcutaneous tissue and differentiated into spontaneously beating cardiomyocytes on a tent-like thin membrane as part of a fluid-driving micropump [21] (Fig. 7). Biohybrid microsystems have emerged within the last decade and have evolved into promising alternatives that concurrently use the actuation and the sensing capabilities of biological cells. Although these systems come with challenges related to increasing lifetime and issues with packaging, they open up promising new pathways, for instance, co-culturing of muscle cells with neuronal cells to recreate neuromuscular junctions for use in microsystems. 


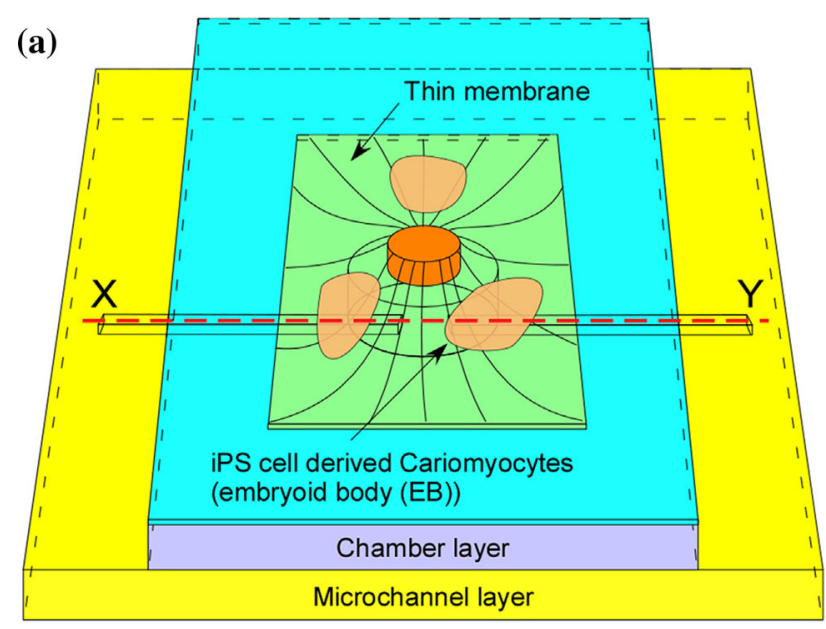

(b)

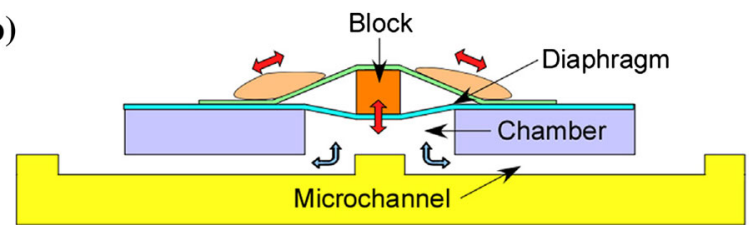

Fig. 7 Design of a membrane-based micropump powered by iPS cells differentiated into cardiomyocytes on a tent-like structure. a Schematic view. b Cross-sectional view along the $X-Y$ axis. From [21], (C2015 Elsevier. Reproduced with permission

Carlsen et al. [22] gave in a thorough review of the latest advances in the field of biohybrid cell-based actuators, including an innovative approach to realizing 3D selfassembled engineered PDMS substrates which are used as enabling matrices to align muscle cells [23], the use of confined bacteria as building blocks to generate fluid flow [24], and the discovery that cardiomyocyte cells are capable of (1) sensing substrate deformations via a mechanosensitive feedback mechanism and (2) dynamically reorganizing themselves [25]. Though bionic concepts can be rated as an exciting pathway for future research, robustness and reliability of the components presented by now is by far not sufficient for real application in microfluidics. Much further developed on the contrary are actuation schemes based on PDMS composites, both with regard to basic understanding and the spectrum of achievable properties.

\subsection{PDMS composite actuators}

By definition, composite materials (i.e., composites) are made of two or more constituents with different physical or chemical properties that, once combined, exhibit properties that differ from those of the individual components. Generally, polymer composites consist of a base polymer and a dispersed phase, typically some kind of particle in the nanometer or micrometer size range. The composites can be micropatterned using conventional microfabrication processes such as micromolding, screen and inkjet printing, and photolithography, and remain compatible with the undoped base polymer, which is a major advantage for integration of the active component into the microfluidic chip. In all actuation concepts based on polymer composites presented so far, one kind of particle has been dispersed. However, the creation of composites consisting of more than two phases, leading to materials that are responsive to multiple stimuli, would also facilitate the realization of complex actuation schemes.

Magnetic Composites Magnetic polymer composites consist of a base polymer and a dispersed magnetic phase, typically solid particles in the size range of a few nanometers to a few micrometers and with specific magnetic properties. They have proved to be versatile in the realization of microfluidic actuators. The adjustable magnetic properties can be diamagnetic, paramagnetic, superparamagnetic and ferromagnetic, depending on chemical composition, crystalline structure and size of the particles used. The most prominent candidates are neodymium $\left(\mathrm{Nd}_{2} \mathrm{Fe}_{14} \mathrm{~B}\right)$ and iron(III) oxide $\left(\mathrm{Fe}_{2} \mathrm{O}_{3}\right)$. A recent review by Gray [26] of magnetic composite materials in microfluidic devices has shown that PDMS is the most common base polymer for creating magnetic composites, and that in most cases composite membranes or cilium-like structures actuated with electromagnets are used for fluid transport and mixing. Typical examples of the membrane category were presented in $[27,28]$, where iron oxide particles were mixed with PDMS in order to fabricate magnetically responsive membranes for pumping and active mixing in microfluidic channels. In [29], a neodymium-based PDMS composite was used to realize a membrane actuator designed for microfluidic pumping with the special feature that the neutral position of the membrane was determined by diamagnetic levitation (Fig. 8).

The same type of composite with different weight ratios of neodymium to PDMS and different degree of magnetization was used to realize purely passive and normally closed pressure-sensitive valves with an adjustable switching point for each individual valve [30]: Flexible composite membranes and magnetized composite blocks are integrated, respectively, above and below a microfluidic channel such that the magnetic attraction between them closes the channel. With increasing pressure in the channel, the valve switches into the open position, where the switching point depends on the weight ratio in the flexible membrane and the strength of the composite magnet underneath. In [31], a method for preparing elastomeric magnetic films with increased responsiveness to magnets was presented. This was achieved by aligning the magnetic particles throughout the thickness of the elastic film during the curing process and subsequent magnetization. The 


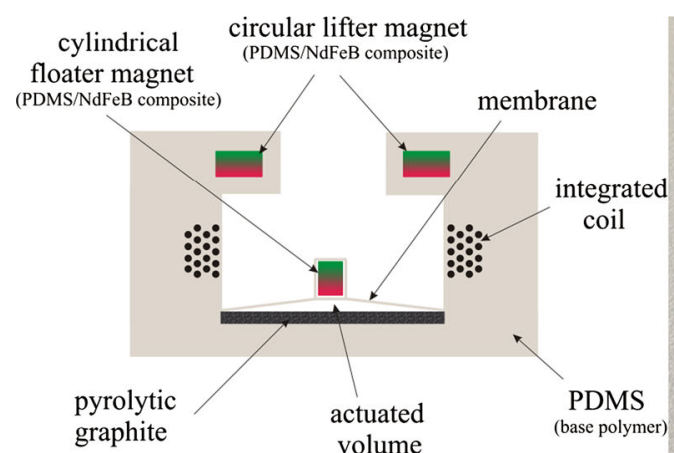

Fig. 8 (Left) Schematic of membrane actuator utilizing the effect of diamagnetically stabilized levitation. (Right) Photograph of the device fabricated in polymer composite technology with PDMS as the base

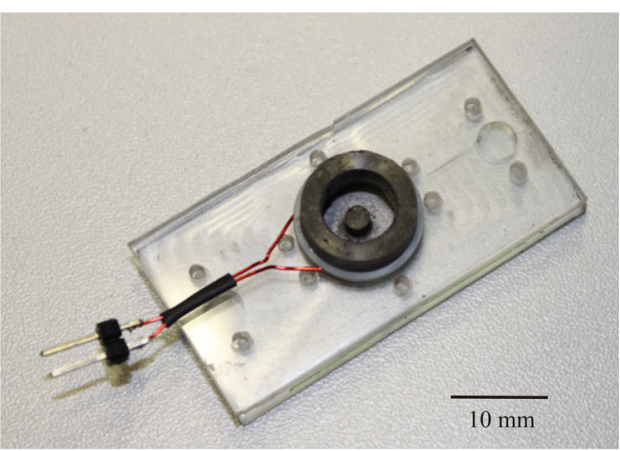

polymer and $\mathrm{NdFeB}$ powder as the magnetic component. From [29], C2013 IEEE. Reproduced with permission

performance and control capabilities. Multiple-step micromolding processes have been applied to create defined cilia with high aspect ratios (up to $\sim 60$ ) and with various ratios of doped to undoped PDMS for active mixing [36, 37]. In [38], a core-shell fabrication method for cilia was suggested which decouples the elastic and structural components such that the actuator response can be optimized and actuation of fluids 550 times more viscous than water becomes feasible. Standard soft lithographic techniques have also been applied to fabricate artificial cilia, but the maximum achievable aspect ratio is relatively small. The authors of [39] used such an approach and focused on the asymmetric movement of the artificial cilia, which is crucial for generating a net fluid flow in the low Reynolds number regime. In short, the cilia were actuated with a rotating magnetic field, and the asymmetry in movement was achieved using remanent magnetization of the cilia perpendicular to the plane of rotation. Only recently have Liu et al. [40] proposed using a 3D extrusion printer to fabricate artificial magnetic cilia with high aspect ratios that are based on PDMS and iron particles. An actuation concept somewhat related to artificial cilia was presented in [41]: A PDMS composite-based cantilever-like structure is fixed to a wall of a microchannel with a slit orifice downstream. Because of the vibrational motion of the valve membrane, the slit orifice is designed to make the flow asymmetric; a net flow in the order of $\mu \mathrm{l} / \mathrm{min}$ is thus induced in the microfluidic channel (Fig. 10). An alternative concept that employs magnetic composites was presented in [42]: Geometrically tailored composite structures embedded in a PDMS-based chip are used for guidance and control of the magnetic flux density in microstructures. This technique was applied for controlled actuation of a small ferromagnetic sphere at the $\mathrm{x}$-junction of two microfluidic channels, which allows the flow in the channel network to be manipulated and controlled in many ways. Another interesting application of magnetic PDMS composites was presented by Peng et al. [43]. They fabricated and 


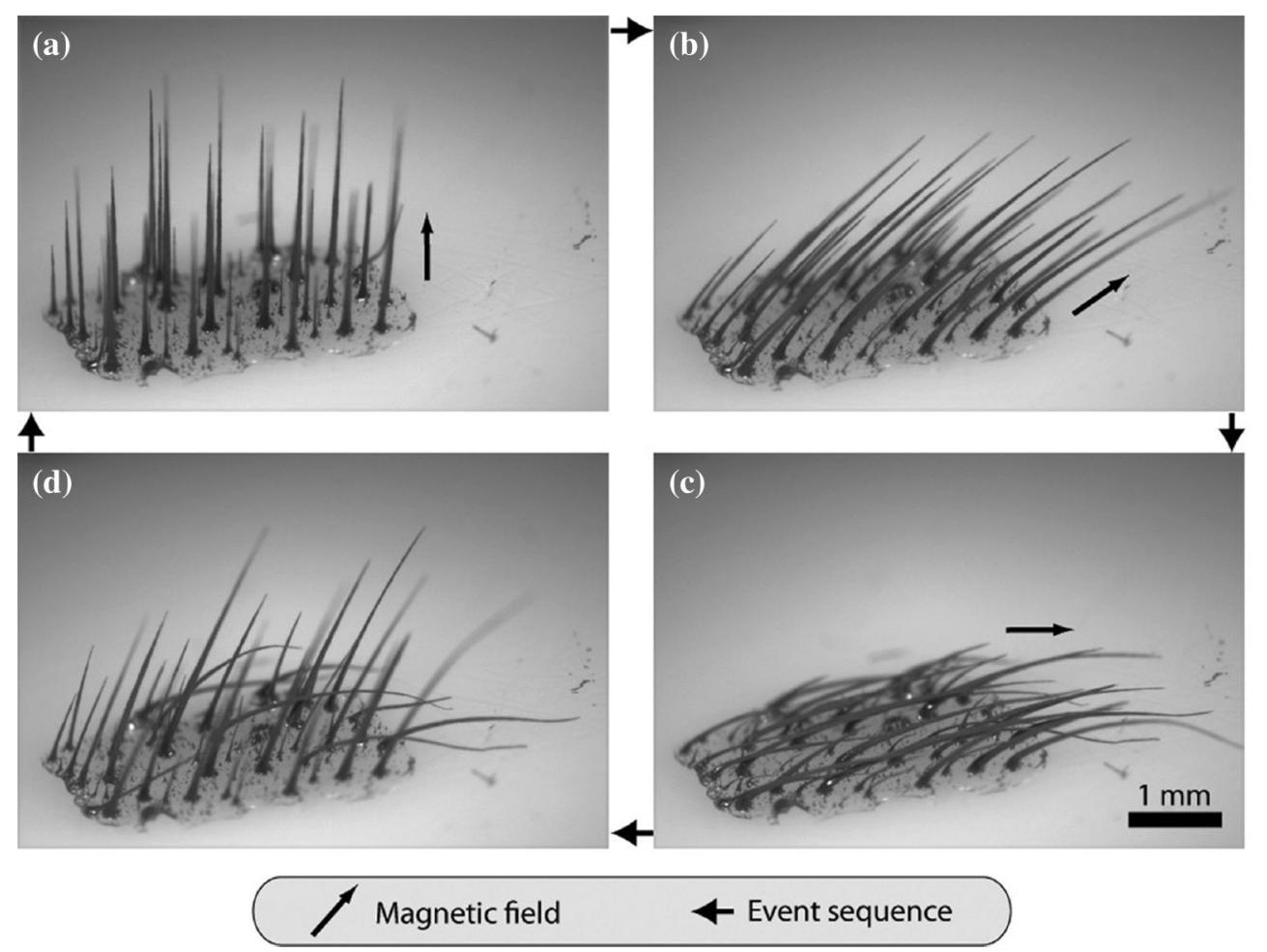

Fig. 9 Magnetic-field-induced bending of artificial cilia made in a 450-mT magnetic field with a $20 \mathrm{~T} / \mathrm{m}$ vertical gradient. The magnetic field is a perpendicular, $\mathbf{b}$ at a $45^{\circ}$ angle, and $\mathbf{c}$ parallel to the substrate

characterized magnetically responsive elastic microspheres, either with core-shell or solid structures, by using a microfluidic flow-focusing device. Although an application in microfluidics has not yet been presented, the magnetoresponsive composite particles may have potential for specifically devised uses in fluid manipulation. Further, direct-writing methods have already been applied to fabricate magnetic composite actuators [44]. Although a specifically tailored monomer mixture was used in place of PDMS as a base, it was successfully shown that inkjet printing of magnetic-non-magnetic polymer composite actuators is possible.

Thermal Composite Actuators Small core-shell microspheres composed of a gas-tight shell containing liquid hydrocarbon and incorporated into an elastomer matrix form a thermally or infrared responsive composite. When heated, the microspheres' thermoplastic shells soften, while the hydrocarbon undergoes a phase change from liquid to gas. Thus, the volume of the microspheres increases, which leads to a considerable irreversible volume change of the composite; the microspheres do not shrink again when the composite is cooled down. Nevertheless, integration of expandable microspheres into a PDMS matrix has been proposed for liquid handling in microfluidic applications, especially for disposable devices surface. d After removal of the magnetic field, the artificial cilia relax toward the surface. From [33], (C2010 American Chemical Society. Reproduced with permission

[45-47]. Very recently, expandable microspheres have been used to develop laser-activated single-use micropumps [48]. Flow rates in the order of $2.2 \mu \mathrm{l} / \mathrm{s}$ and total volumes over $160 \mu \mathrm{l}$ were achieved. The combination of these core-shell microspheres with carbon nanotubes (CNTs), both dispersed in a PDMS matrix, results in a composite material with a binary set of material properties (Fig. 11). Again, upon thermal or infrared stimulation, the material undergoes visible dimensional changes, but additionally the conductive nanotubes in the polymer matrix exhibit a load-sensitive electrical response and thus allow remote expansion monitoring [49]. Further, pure CNT networks within the silicone elastomer have been used to fabricate electrothermal actuators with a visible maximal strain of $4.4 \%$ at an electric power density $0.03 \mathrm{~W} / \mathrm{mm}^{-3}$ [50].

Electroactive Composites Uses of electroactive PDMS composites in microfluidics are essentially limited to acting as conducting composites in the fabrication of flexible electrode structures [51, 52]. PDMS can be turned into a conductive material by adding, for instance, carbon black or metal powder in specific weight percentages in order to achieve good electrical conductivity [53] and tuned elastic properties [54]. Composites with multiwalled carbon nanotubes (MWNTs) were used in [55] to develop a 
Fig. 10 (Left) Schematic diagram of the pumping principle using a vibrating valve: a top view, b 3D view with dimensions, and c schematic actuation. (Right) Scanning electron microscope images of the fabricated PDMS structure: a bird's eye view, b $3 \mathrm{D}$ view, and $\mathbf{c}$ front view. From [41], (C2012 Springer. Reproduced with permission
Vibrating valve including magnetic particles

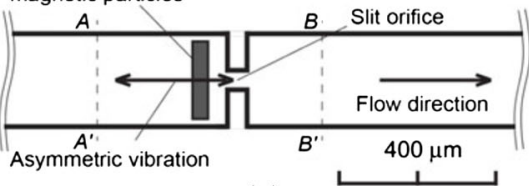

(a)

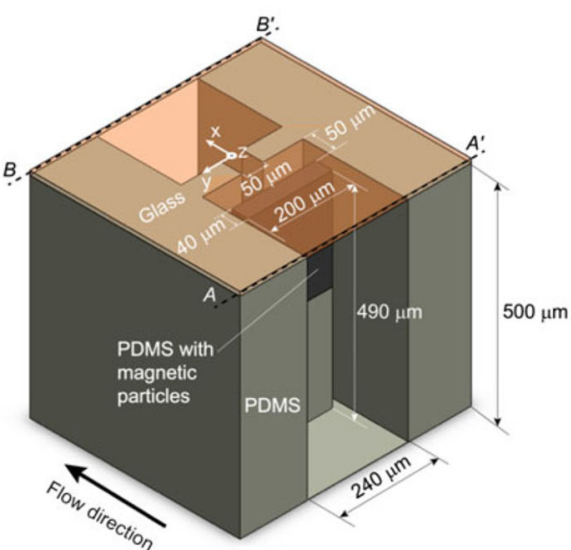

(b)

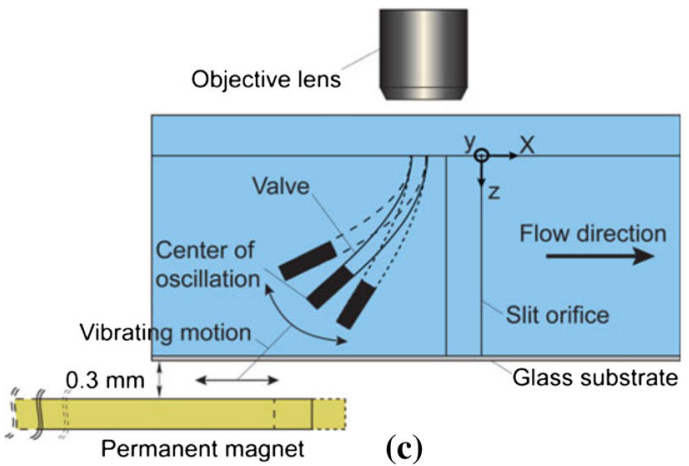

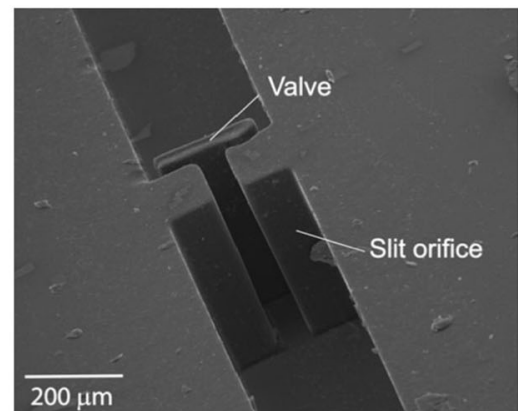

(a)

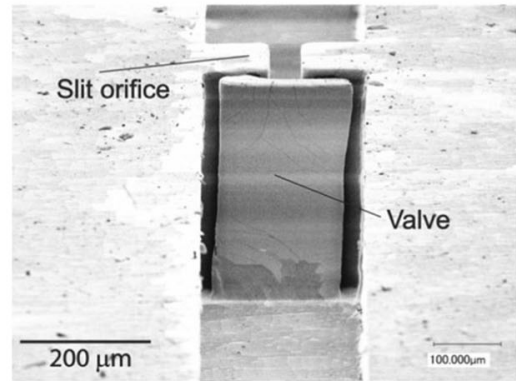

(b)

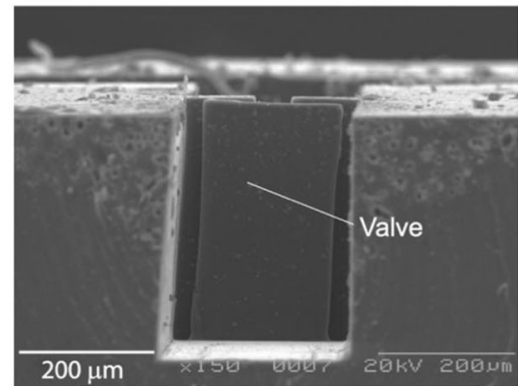

(c)

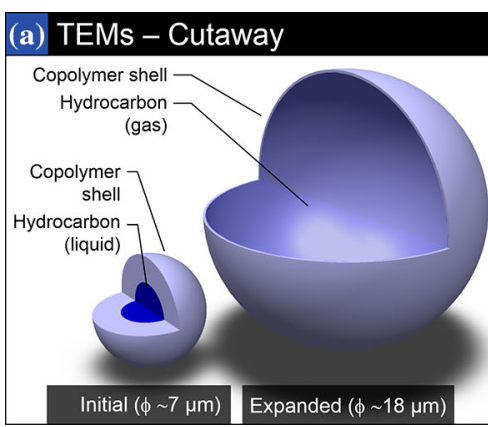

Fig. 11 Thermally expanding core-shell microspheres: a To scale cutaway showing size relationship between initial and expanded microspheres. b Scanning electron microscope (SEM) image of loose, unexpanded microspheres (average diameter of $7.1 \pm 1.9 \mu \mathrm{m}$ ).

monolithic electrostatic actuator that scales at a rate of $\sim 100$ actuators per $\mathrm{cm}^{2}$. Integrated into a microfluidic device, they were used to regulate microchannels pressurized at up to 40 mbar with operating voltages below $300 \mathrm{~V}$. Actuation of carbon nanotube (CNT) assemblies can be

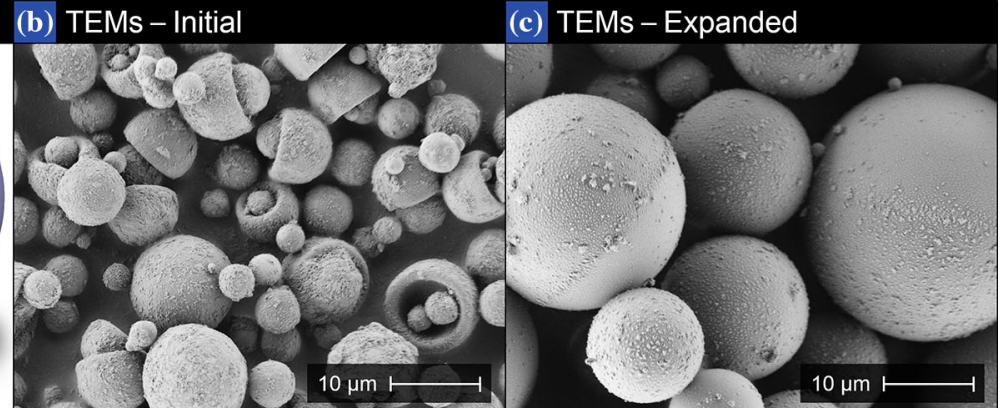

c SEM of loose, expanded microspheres (average diameter of $17.8 \pm 3.8 \mu \mathrm{m})$. From [49], (C2013 IOP Publishing Ltd. Reproduced with permission

achieved by electrochemical charging where the CNTs are in contact with an electrolyte. The mechanism is based on non-faradaic double-layer charging, which changes the carbon-to-carbon bond length. Cantilever-like actuators have typically been realized either in direct contact with a 
liquid electrolyte or as a multilayer structure with organic electrolytes, and reviews of such concepts are given in $[56,57]$. Another interesting application in microfluidics was described in [58], where conductive composites and fluids with giant electrorheological properties were combined to realize a microfluidic logic switch and inverter in a single microfluidic chip.

\subsection{Layered PDMS structures}

Layered Bistable PDMS Structures Bistable actuation concepts are particularly interesting for microfluidic applications because they require energy only during transition between two stable states, which is ideal for realizing microfluidic valves. Around 1995, the idea of using buckled microstructures for actuation was introduced, and in [59] a bistable diaphragm composed of polyimide and a silicone platelet, integrated into a PMMA housing, was used to open or close the inlet of a microvalve. Thermopneumatic actuation was achieved using an integrated resistive heater. The same group published theoretical work that aimed to derive simple equations which allow the basic features of these valves to be described, and an optimized design for specific applications [60]. A phase-change actuator, using n-pentane as a working fluid, that enables abrupt and large volume changes and thus reduces switching times of the elastic bistable diaphragm was suggested by [61]. A further development of this actuation concept was presented in [62], where a thermopneumatically actuated bistable microvalve was developed with reduced sensitivity (1) to the temperature of the fluid to be switched and (2) to sticking. This was achieved by design considerations and the integration of small permanent magnets that keep the diaphragm in its stable positions. Switching times as short as $10 \mathrm{~ms}$ with a minimum energy consumption of $320 \mathrm{~mJ}$ per switching event were achieved. Finally, a manually controllable variant designed for integration into pneumatically powered microfluidic networks for on-demand point-of-care applications was presented in [63]. The authors demonstrated the clinical relevance with a diagnostic chip that processes whole blood samples for blood-type identification.

PDMS-based Dielectric Elastomer Actuators (DEA) DEA actuators, also referred to as artificial muscles, consist of a thin elastomer membrane sandwiched between two compliant electrodes. If a voltage is applied across the two electrodes, the opposite charges on each electrode give rise to an electrostatic force which induces Maxwell stress and squeezes the dielectric layer, thus deforming the device [64]. Although the applicability of a DEA actuator as the motive element of a potential prosthetic blood pump was investigated a decade ago [65], DEAs have so far hardly been employed for actuation in microfluidics, most probably due to the rather small achievable strain in these structures and the high voltages (in the order of some $\mathrm{kV}$ per $\mathrm{mm}$ ) required to achieve sufficient deformation. Loverich et al. [66] developed a concept for an all-polymer micropump with DEA fluid actuation and integrated check valves to rectify the fluid movement (Fig. 12). They achieved a maximum flow rate and back pressure of $77.4 \mu \mathrm{l} / \mathrm{min}$ and $8.45 \mathrm{kPa}$, respectively. Other examples are the utilization for active tuning of channel geometry [67], the incorporation as an active valve into a microchip [68], or the realization of DEA-based tunable lenses [69-71]. However, Lamberti et al. [72] presented a comprehensive study on the durability of the electromechanical properties of PDMS for use in DEA actuators. They showed that by variation of the mixing ratio between oligomer base and the cross-linking agent the achievable Maxwell stress can be influenced considerably: they measured a $150 \%$ increase at $1 \mathrm{~Hz}$ actuation frequency for a 20:1 mixing ratio. In combination with multilayer designs that consist of stacked layers of elastomer and electrodes, electromechanical transducers with reasonable displacements can be developed which may have great potential for use in microfluidics. The authors of [52] developed beam-shaped multilayer microactuators consisting of three conductive and two dielectric elastomer layers, fabricated in a micromolding process. Application of a voltage across two of the neighboring electrodes induces asymmetrical axial strain and thus bending of the beam. They achieved a tip displacement of $318 \mu \mathrm{m}$ for a $100-\mu \mathrm{m}$-wide, $40-\mu \mathrm{m}$-thick, and 1-mm-long actuator at $1.1 \mathrm{kV}$ actuation and $10 \mu \mathrm{W}$ electrical power consumption. An analytical model for the optimization of DEA-based multilayer stack transducers has recently been published [73]. The authors derived equations for finding the settings that lead to optimal conversion of electrical input energy to mechanical work. Another way to overcome the inherent weakness of relatively small achievable strains of DEA-based actuators was presented in [74], where two DEAs were used to provide the actuation force for switching between two equilibrium positions of a bistable minimum-energy structure. In this configuration, large deformations were achieved, and the actuation concept was proposed as a building block in robotic applications. Although the issue of integrating high voltage into a microfluidic chip remains, the dimensions and actuation amplitudes are already at a scale that is feasible for microfluidics. On the materials side, it has just been shown that by cross-linking a few weight percent of azobenzene with hydroxyl-terminated PDMS yields a silicone rubber with considerably increased electromechanical actuation performance [75]. The maximum actuation strain achieved was three times higher than for unmodified PDMS. Further, first findings concerning the temporal evolution and instability effects in dielectric elastomer actuators have been published [76].

Electromechanical PDMS membrane actuation Electromechanical actuation of thin PDMS membranes utilizing the electrostatic attraction of oppositely charged electrodes is 
Fig. 12 DEA-based micropump. (Left) Membrane actuator consisting of a balloonshaped structure formed by two layers of dielectric elastomer (a). Electrostatic compression of the lower membrane caused the prestrained diaphragm to move downward (b). (Right) Fabricated pump consisting of the DEA actuator and two check valves. From [66], (C2006 The Royal Society of Chemistry. Reproduced with permission (a)

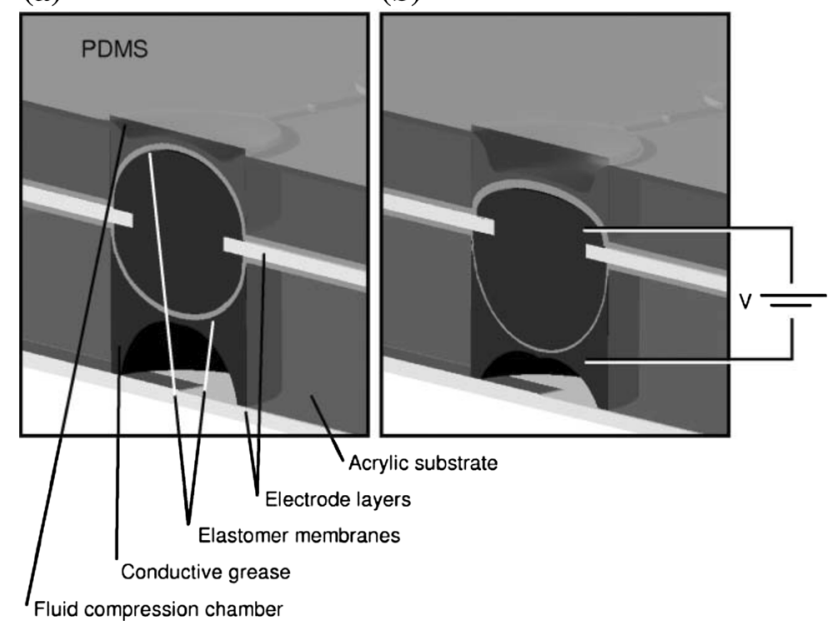

(b)

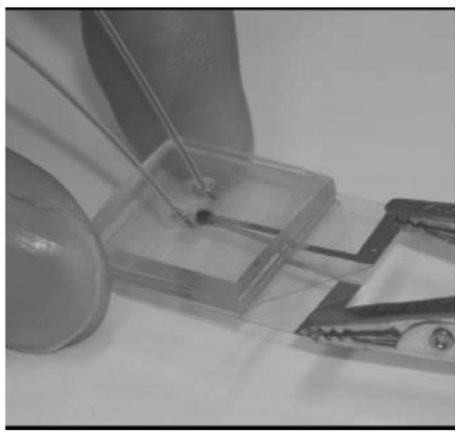

much less common, most likely due to the relatively high voltages necessary to achieve adequate strain for valve or pump applications. Another issue is the fabrication of reliable electrodes on the PDMS surface. Realizing highly conductive metal films requires processes such as vacuum deposition, chemical etching and high-temperature steps which may degrade, or at least alter, the elastomer. Moreover, metal films tend to buckle under strain of the actuated elastomer. Possible ways of overcoming this issue are (1) using electrically conductive composites, as described above, at the cost of reduced electrical conductivity and (2) devising specifically tailored channel geometries with very small sidewall angles to prevent the metal layer from fracturing [77]. Another way to prevent cracking of the metal electrode is the integration of thin-film metal flexures with, for example, helical shape into the flexible membrane, as described in [78]: Using this approach, the authors realized a reconfigurable microfluidic device consisting of a number of valve chambers, each of which is connected to adjacent chambers and independently electrostatically actuated. This allows the device to be configured for pumping, mixing, spitting, or circulating flow (Fig. 13). In [79] an electrostatic microvalve was developed which was used to regulate pressures in hydraulic control lines, which in turn were used to actuate pressure-driven components, e.g., microvalves. The same group published design considerations for electrostatic microvalves in PDMS-based microfluidics [80]. In [81] a normally closed electrostatic valve structure was demonstrated which isolates the fluid from the electric field, hence featuring relatively low actuation potentials $(<60 \mathrm{~V})$, and in [82] an electrostatic valve with liquid metal-elastomer-compliant electrodes was presented. A brief overview of the different techniques for making stretchable metal interconnects on polymers was given in [83]. Kim et al. [84] used an electrically conductive polymer in place of metal electrodes to actuate PDMS membranes
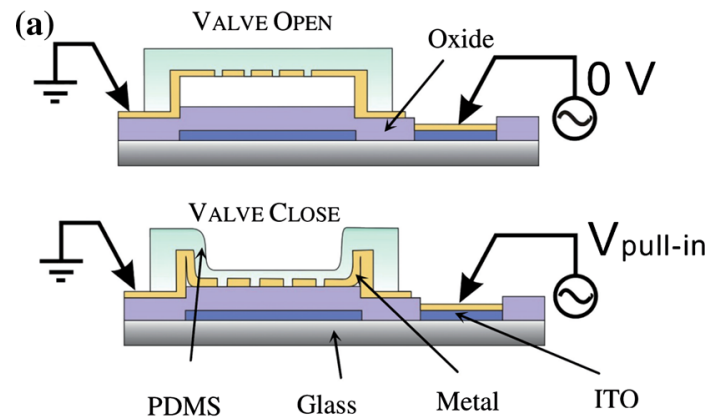

(b)

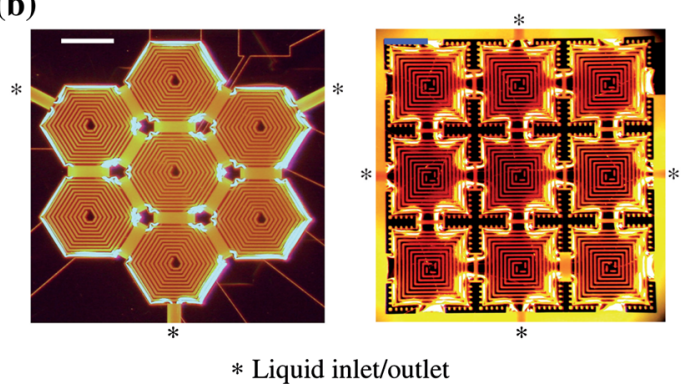

Fig. 13 a Operation principle of normally open electrostatic valve. The top metal flexure and the bottom indium-tin-oxide (ITO) layer form the capacitor plates. b Fabricated valve arrays, hexagon and square shape. By varying the sequence of actuation signal each valve can function as microchannels, valves and pumps. Scale bar, $200 \mu \mathrm{m}$. From [78], C2009 The Royal Society of Chemistry. Reproduced with permission

electromechanically. A sandwich structure of PDMS and the conductive polymer polypyrrole (PPy) was used to realize a microfluidic pump, and actuation was achieved by electrochemical oxidation and reduction of the PPy layer, which causes it to swell or contract, thus moving the diaphragm. In combination with check valves, a micropump was produced with a maximum flow rate of $52 \mu \mathrm{l} / \mathrm{min}$ at remarkably low operating voltages in the order of $\pm 1.5 \mathrm{~V}$ and low power consumption in the order of $55 \mathrm{~mW}$ per stroke. 


\subsection{Microstructures based on PDMS}

Cellular PDMS Structures All actuator concepts presented so far have in common that the PDMS or the composite is solid (i.e., without voids). An interesting technological approach realizes cellular PDMS with micrometer-sized voids to obtain piezoelectric PDMS films for MEMS transducers [85]. By plasma polarization, bipolar charges are implanted in the voids on opposite inner surfaces (Fig. 14). These implanted charge pairs serve as dipoles that respond to electromechanical stimulation. This material can be classified as a so-called electret-a dielectric material which exhibits a quasi-permanent electric charge or dipole polarization. Although no application in microfluidics has so far been reported, it has been used for sensing and energy harvesting [86], and the material may also be suitable for actuators. An open-pored PDMS foam with a gradient in pore size has been used for simultaneous filtration and pumping of fluids [87]. This foam together with a small ring magnet for actuation was integrated into a membrane. Due to the vibrational motion of the membrane
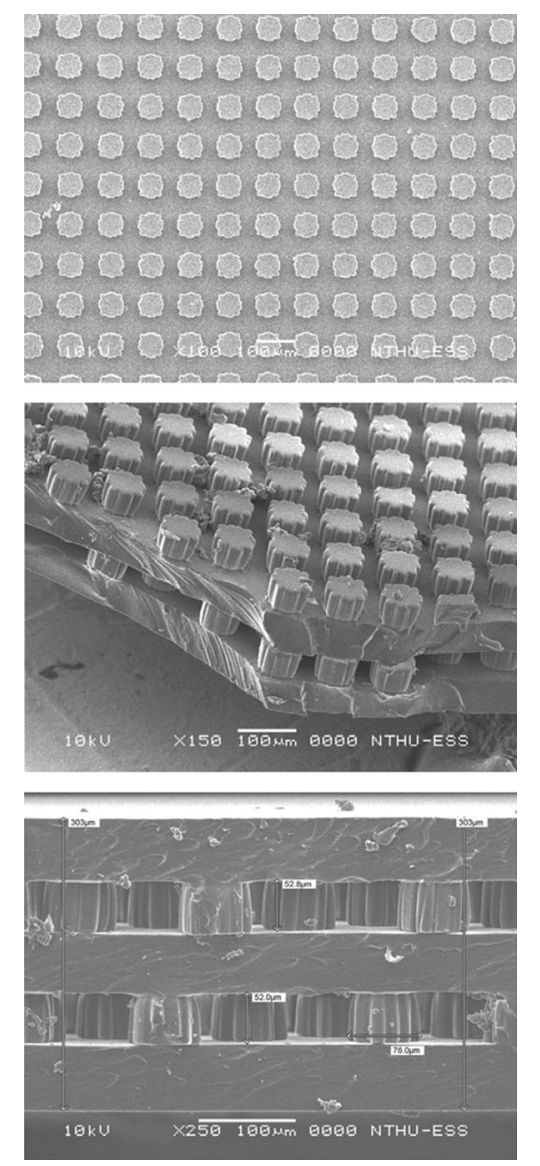

Fig. 14 Scanning electron microscopy photographs of the piezoelectric PDMS films. From top to bottom: top view, 3D view, and crosssectional view of the fabricated cellular structures. From [85], (C2012 IOP Publishing Ltd. Reproduced with permission in the fluid and the gradient in pore size of the foam, which constitutes in essence a nozzle-diffuser structure, a unidirectional flow through the membrane evolves. Concurrently, the fluid is filtered, and the vibrational motion of the filter membrane suppresses fouling and clogging (Fig. 15). Pressure-Induced Actuation Tilted sharp-edged PDMS structures attached to the sidewalls of microchannels are used to actuate fluids by acoustic streaming effects [88]. A standard piezoelectric transducer attached to the microfluidic chip was employed to acoustically oscillate the sharpedged structures in order to generate acoustic streaming effects around their tips. This concept enables flow rate tuning across a wide range, from nanoliters to microliters per minute, simply by adjusting the input voltage of the piezo-transducer (Fig. 16). The same actuation principle was used in [89] for biomimetic silicone cilia. Oh et al. developed an in liquid fabrication method in order to avoid collapsing of the high-aspect-ratio silicone cilia. Rotational and propulsive flows were generated with the cilia in water when resonated by a piezotransducer. The same group adapted this concept for the task of mixing in standard microwell plates [90]. The authors of [91] focused on modeling and simulation of the cilia movement in order to understand the underlying physics and to suggest design parameters for optimal cilia-based micromixers. One of the central findings was that the flow pattern generated by the tips of the cilia changes from one large vortex to multiple small vortices as the spacing between the single cilia exceeds a critical geometry-dependent value. They experimentally proved the concept by employing their ciliabased micromixer to improve bioreaction efficiency of a biotin-avidin assay and a DNA hybridization assay [92]. Pressure-induced actuation of cilia with pneumatically actuated waves underneath a ciliated wall [93] and with pneumatic pipes directly integrated into the cilia [94] have recently been reported. In the latter case the cilia-like actuators have relatively large dimensions, $1 \mathrm{~mm}$ diameter and $8 \mathrm{~mm}$ length, but the fluid speeds measured reached correspondingly large values in the order of $19 \mathrm{~mm} / \mathrm{s}$.

PDMS membranes with microscopic incisions have been reported for use as valves with flow-rectifying properties and the additional possibility to actively control the flow rate in the forward direction [95, 96]: An elastic membrane with an incision is clamped between two rigid polymer plates and expands when pressure is applied in the forward direction, allowing fluid to pass. In the backward direction, expansion of the membrane is prevented, and thus fluid flow is blocked. The authors of [97] discussed design considerations related to actuation pressure minimization, reliability and convenient integration into complex microfluidic devices of such (normally closed) elastomeric valves. The concept of incised PDMS membranes has recently been developed further by [98] for the 


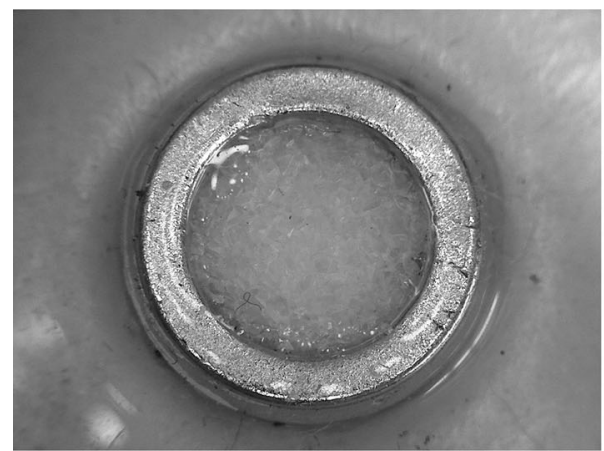

Fig. 15 Microfluidic pumping concept utilizing an open-pored foam with gradient in pore size: (Left) photograph of the foam integrated into the central part of a movable membrane; (Right) sketch of the
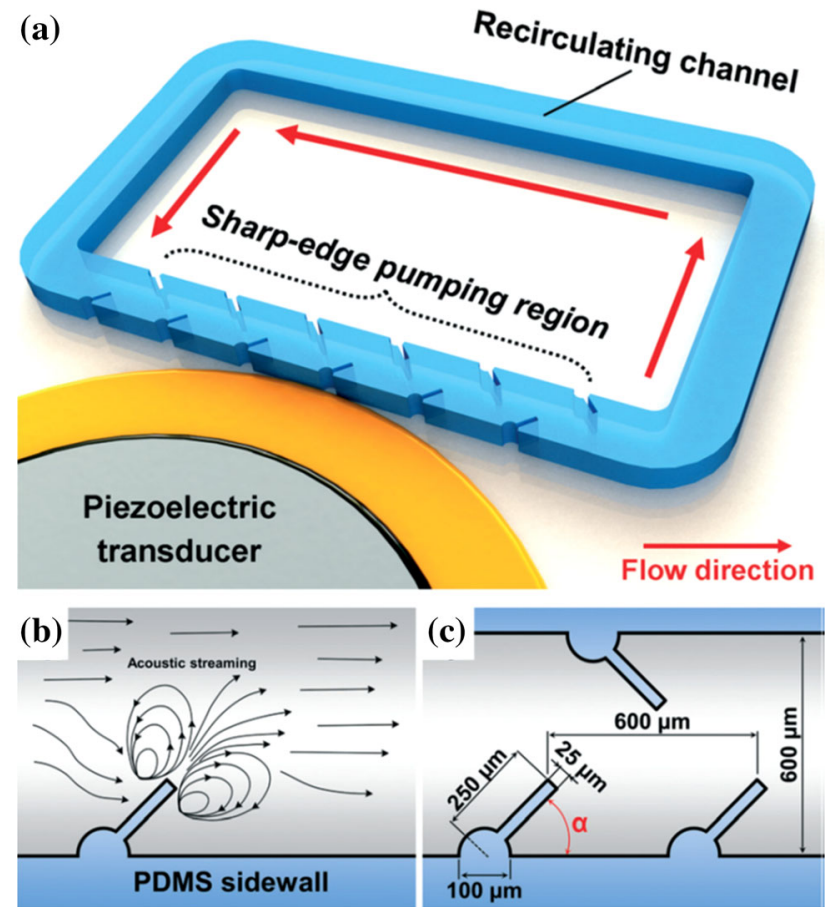

Fig. 16 a Schematic of the sharp-edge-based acoustofluidic pumping device comprising a PDMS microfluidic channel and a piezoelectric transducer. b Schematic of the acoustic streaming phenomenon around the tip of a tilted oscillating sharp-edge structure. c Schematic showing the design of the channel and sharp-edge structure. From [88], C2014 The Royal Society of Chemistry. Reproduced with permission

design and fabrication of artificial sphincters made of elastomeric polymers. Although intended for biomedical applications (the aim was to tackle urinary incontinence), the concept may also have potential in microfluidic structures. By varying the valve's elastomeric stiffness (weight ratio of monomer to curing agent) and its thickness, they were able to tune the opening pressure across a wide range.

A new type of polymer actuator where electroosmotic pumping is used to create hydraulic pressure and hence

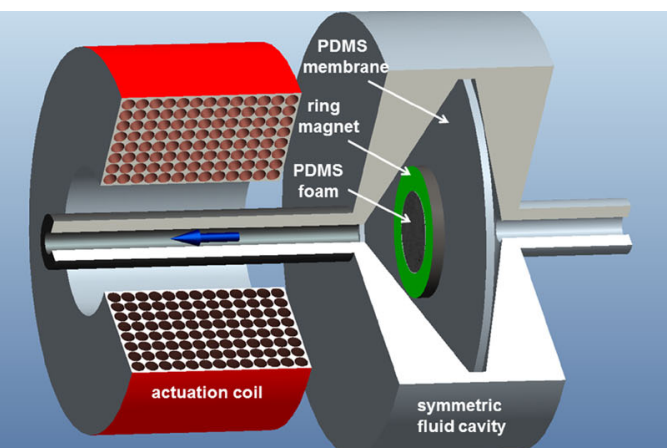

actuation principle with the membrane placed into a fluid cavity. From [87], (C2015 IEEE. Reproduced with permission

membrane deformation in a PDMS-based device was presented in [99]. In analogy to botany where changes in hydrostatic cell pressure, e.g., water content, cause movement of plants, these devices were named 'nastic actuators.' Only recently the same group published a further development, where propylene carbonate (PC) was used instead of water in order to avoid bubble generation and pressure fluctuations which allowed the realization of a fully sealed microfluidic hydraulic actuator [100].

\section{Polymer hydrogels}

So-called polymer hydrogels (PHs) are three-dimensional polymer networks with the ability to store massive amounts of water-up to 99 weight percent of the hydrogel mass. Changes in the proportion of water in the polymer network lead to considerable volume changes, which can reach a factor of 10 or even more. A set of external stimuli such as temperature, irradiation, light, electric and magnetic fields, $\mathrm{pH}$ value changes and ionic strength of the surrounding medium may be suited to inducing changes in the stored water content, which leads to almost instantaneous shrinkage or swelling of the polymer. Thus, the behavior of PHs resembles the hydromorphic motion of plants, which have the ability to bend and wind in response to changes in the amount of water stored in their tissues. Obviously, hydrogels can be operated only in aqueous media, which, on the one hand, limits their potential use as transducers, but is on the other hand ideal for microfluidics. Since Beebe et al. introduced PH structures for autonomous flow control inside microfluidic structures in 2000 [101], their publication has been cited over 1000 times, and hydrogel-based actuator schemes are among the most widely published basic approaches. Several review papers address hydrogel-based actuators in the context of (1) general issues concerning possibilities and limitations [102], (2) their mechanical deformation behavior [103], and (3) their application in 
microfluidics [104-106]. This review aims to highlight concepts specifically devised for use in microfluidics that may possess high potential for future applications.

\section{1 pH-sensitive PHs}

In their pioneering work, Beebe et al. [101] introduced a technique for in situ fabrication of active hydrogel components inside microfluidic channels via direct photopatterning of a liquid phase (Fig. 17). Further, they demonstrated significantly improved response times to $\mathrm{pH}$ value changes in the order of $10 \mathrm{~s}$ and below, which had made hydrogel actuators interesting for use in microfluidics in the first place. Polymerizing the hydrogel structures around prefabricated supports, in this case posts, yielded geometrically stable objects and short response times owing to short diffusion paths of the hydrogel jackets. Later, the same group published a paper focusing on a variety of methods that are based on the inchannel photopolymerization technique [107]. They also demonstrated a so-called 3D hybrid valve, where the regulating $\mathrm{pH}$ solution is not in direct contact with the sensing fluid, but separated by a flexible PDMS membrane. The concept of photopolymerization of small hydrogel structures was also used by Park et al. $[108,109]$. In-flight polymerization of hydrogel droplets, generated by means of a microfluidic flow-focusing device, was used to create hydrogel microspheres which were then employed as an actuating element in a PDMSbased microfluidic apparatus. Chitosan-based hydrogels for microfluidic switching applications were developed with the fundamental advantage that the valving responds to a small range of $\mathrm{pH}$ values [110]. The previously discussed cilia-inspired actuation scheme has also emerged in connection with hydrogels. In [111], the swelling and shrinkage behavior of hydrogel micropost and microfins was utilized to mimic cilia-based fluid transport in microfluidic structures in response to $\mathrm{pH}$ value changes. In their work, they used electrochemically generated $\mathrm{pH}$ gradients to visualize the hydrogel volumephase transition and how it translates into movement of the microstructures. The authors of [112] realized a gel membrane whose pore size, permeability, adhesive and mechanical properties can be regulated via the $\mathrm{pH}$ and ionic strength of the surrounding medium. Additionally, they demonstrated that the membrane can also store nanoparticles and enzymes, which enables its use as antimicrobial barrier and microreactor capable of metabolizing and emitting chemical substances. Yang et al. [113] combined polymeric hydrogels with porous membranes and thus realized composites with promising functionality, for example, as stimulus-responsive membranes, highly efficient absorbers for separation and purification, porous membrane valves, and microfluidic gates. The authors focused mainly on $\mathrm{pH}-$ and temperature-sensitive gels, but of course these 'smart' membranes can also be designed to respond to any other stimulus. The concepts presented so far utilize changes in the $\mathrm{pH}$ value of the surrounding medium to induce movement of the hydrogel structures; in [114], an alternative approach was presented: The authors used a phototriggered $\mathrm{pH}$ jump reaction of an integrated protonreleasing agent to induce local changes in the $\mathrm{pH}$ value within the gel structure and thus reversible movement. Although the concept is insufficiently robust-after a few actuation cycles, the bending capability decreased-and it has not yet been applied to microfluidics, it may be an interesting approach for microfluidic technologies. On the theoretical side, studies have been published that computationally explore (1) the actuation behavior of pH-sensitive PHs for different microfluidic channel and hydrogel geometries [115] and (2) the deformation behavior of a $\mathrm{pH}$-sensitive hydrogel microfluidic valve system in relation to fluid-structure interactions [116]. The authors described strategies for improving the response behavior of $\mathrm{PH}$ actuators in general (not only that of $\mathrm{pH}$-sensitive ones), and provided models for the optimized design of hydrogel-based actuators.
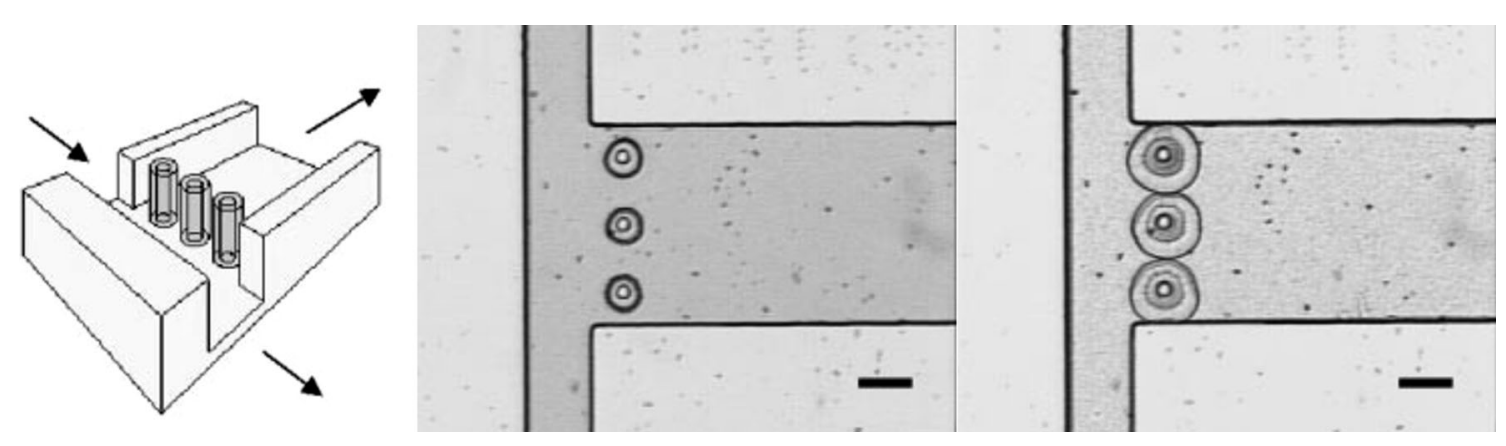

Fig. 17 (Left) Schematic of microchannel (T-junction) with prefabricated posts serving as supports for the hydrogels. (Middle) Device after polymerization of the hydrogel. (Right) The hydrogel jackets, stimulated by $\mathrm{pH}$ value changes, block the side channel. From [101], (C2000 Macmillan Publishers Ltd. Reproduced with permission 


\subsection{Photosensitive PHs}

Light-sensitive hydrogels have attracted attention because they can be stimulated remotely and precisely. The physical mechanisms for photoinduced deformation of polymer hydrogels include changes in cross-link density, intramolecular group or hydrogen transfer, dissociation processes, cis-trans isomerization, and pericyclic reaction. However, these processes are relatively slow and therefore generally not well suited for typical microfluidic actuation [117] (Fig. 18). In order to exploit the advantages of stimulation by light, infrared radiation sources and temperature-sensitive hydrogels have been used to fabricate high-pressure-resistant microvalves based on poly(N-isopropylacrylamide) (PNIPAAm) [118]. In their work, the authors found that the pressure tolerance of the gel can be

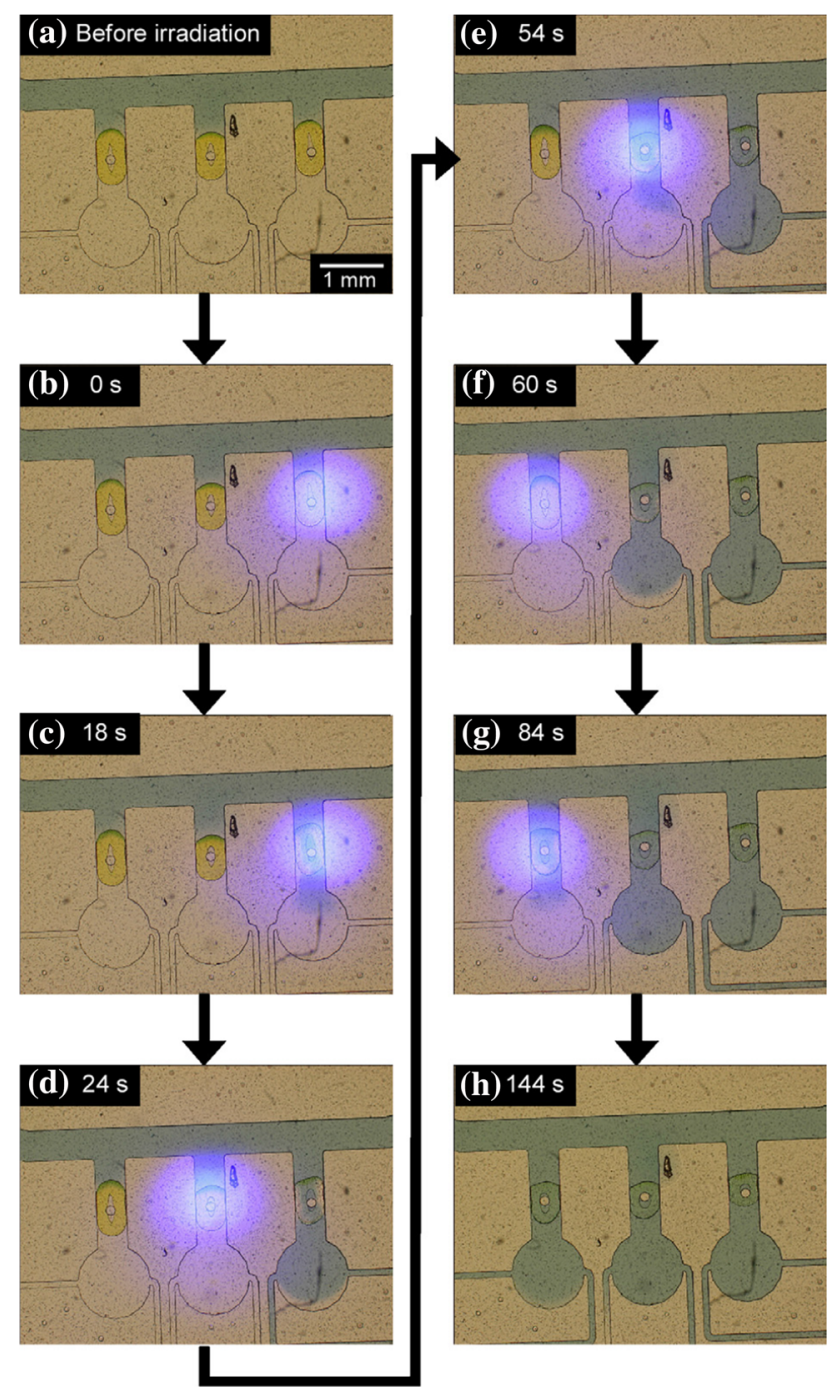

Fig. 18 Independent control of multiple pSPNIPAAm gel microvalves by means of local light irradiation. From [117], (C)2007 Elsevier. Reproduced with permission tuned by choosing suitable amounts of monomer and crosslinker for the polymer monolith inside the microchannel. Microvalves were fabricated that withstand leakage at pressures up to 93 bar and that feature opening and closing response times in the order of only a few seconds. The integration of light-absorbing particles or molecules into a temperature-sensitive hydrogel has led to gel structures with even faster swelling or shrinking properties. Nanocomposites of elastin-like polypeptides and graphene sheets have been developed and feature response times in the order of seconds or even less and improved control over the location of actuation [119]. In [120], the photothermal deformation mechanics of a temperature-sensitive hydrogel impregnated with light-absorbing nanoparticles was studied theoretically by considering the equilibrium thermodynamics of a swelling gel using a variational approach; additionally a case study in which the composite was employed as a microvalve was presented. Jadhav et al. [121] used a composite of PNIPAAm and polypyrrole (PPy) nanoparticles to realize a photoresponsive microvalve for remote actuation with NIR-laser irradiation for flow control in microfluidic devices.

\subsection{Temperature-sensitive PHs}

The vast majority of temperature-sensitive PHs in microfluidics is used for flow rate control or valving applications. Either the $\mathrm{PH}$ structure is directly integrated into the microfluidic channel to achieve blocking and releasing functionality upon temperature change [122-125], or the $\mathrm{PH}$ actuator is separated from the fluid channel by an elastomer that squeezes the channel [126, 127]. In [128], an analytical and numerical study of the inhomogeneous swelling behavior of the temperature-sensitive hydrogel PNIPAAm was presented and verified by experimental results, which is valuable for the design of hydrogel-based microvalves. For active fluid transport (i.e., pumping applications), there are again two ways of using $\mathrm{PH}$ inside microfluidic structures: either in diffusion mode, where the swollen hydrogel is heated and thus releases the fluid directly into an outlet to generate pumping pressure, or in displacement mode, where the gel is placed into a separate actuation layer and exerts pressure on an elastic membrane (Fig. 19). Richter et al. [129] fabricated both types using PNIPAAm and simple resistive heaters integrated into the structure as control elements. Based on the maximum operating parameters (flow rate and back pressure), they concluded that the diffusion type is well suited to low-performance applications, whereas the displacement type is better suited to medium- or high-performance applications. The same actuation principle-integrated resistive heaters and thermally responsive PNIPAAm in a microfluidic channel-was also used to realize valves that are normally closed at room temperature [130]. Upon heating above the lower 


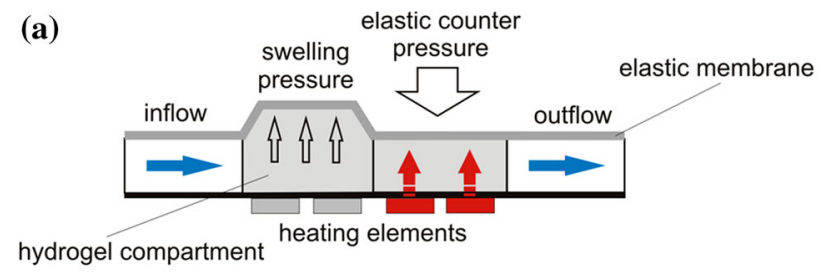

(b) swelling agent supply

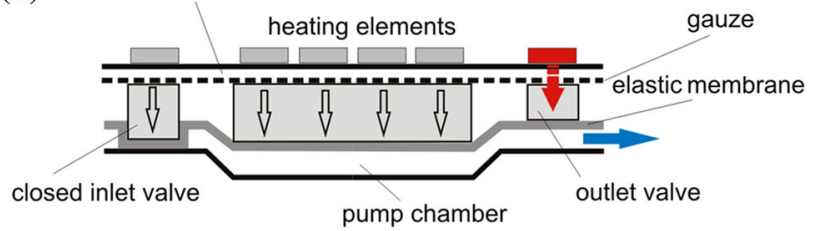

Fig. 19 Schematic setup of hydrogel-based micropumps. a Diffusion micropump. b Displacement micropump

critical solution temperature (LCST), the gel becomes hydrophobic, shrinks and forms pores which allow the solution to flow. A concept where the temperature-sensitive hydrogel was used indirectly to realize programmable autonomous micromixers and micropumps was presented in [131]: The gel operates as a temperature (and/or $\mathrm{pH})$-sensitive clutch which controls the movement of magnetically driven rotors integrated into a microfluidic device. The $\mathrm{pH}-$ and temperature-sensitive hydrogel poly(DMAEMA-HEMA) is used as a clutch which stops the rotation of an externally driven nickel impeller when the temperature is decreased, and starts rotating again when the temperature increases. The rotating impeller induces a pressure change inside the channels which provides the force for driving the fluid through the network (Fig. 20). Lin et al. [132] designed an in situ fabricated hydrogel microvalve for thermostatic control within a microchip. The PNIPAAm-based hydrogel valve enables autonomous on/off switching triggered by on-chip

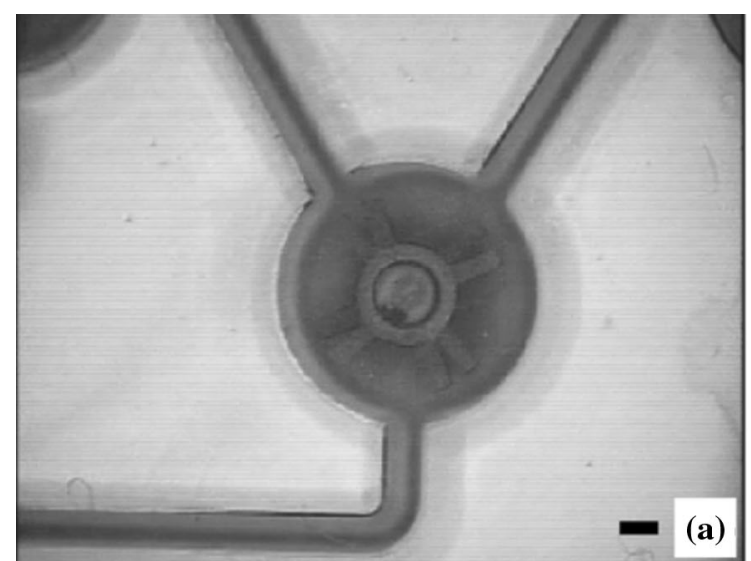

Fig. 20 Four-blade Ni rotor showing the feasibility of controlling fluid mixing at the microscale using poly(NIPAAm) hydrogel rings. Scale bars represent $1.0 \mathrm{~mm}$. a In-flow of warm water causes the poly(NIPAAm) ring to shrink, allowing the four-blade Ni rotor to temperature variations in order to control fluid flow and thus the associated heat exchange. Hence they demonstrated temperature self-regulation within a constant range, which is crucial for microchip systems in the biomedical field. Surfaces that can change reversibly from super-hydrophobic to superhydrophilic upon temperature variation haven been realized by integrating silanized particles into the hydrogel [133]. On the materials side, it has been shown that an ionogel, comprising PNIPAAm and the ionic liquid 1-ethyl-3-methylimidazolium ethyl sulfate, has superior actuation properties compared to the pure hydrogel [134]. The advantages reported were (1) that the tendency of standard hydrogels to become brittle when stored under ambient conditions was reduced due to the low vapor pressure of the ionic liquid and the associated ability to plasticize the gel structure and (2) that the swelling and shrinking properties were improved considerably, which was demonstrated experimentally by means of an ionogelbased microvalve. Another interesting material effect used to realize a thermosensitive polymeric actuator was presented in [135]. The authors discovered a reversible actuation effect of poly(caprolactone) (PCL) — gelatin bilayer films upon temperature variation: the structure of the hydrophobic PCL switches reversibly upon melting and crystallization. Although this behavior is not yet well understood, the actuation concept may also have potential in microfluidic applications. 3D printing of thermally responsive hydrogel structures with specifically devised inks has been demonstrated using the example of a smart valve for flow control [136].

\subsection{Magneto-active PHs}

A composite of PNIPAAm and magnetic iron oxide $\left(\mathrm{Fe}_{3} \mathrm{O}_{4}\right)$ nanoparticles was used by [137] to fabricate magnetic-field-controlled microfluidic valves: The nanocomposite gel is integrated into the channel of a low-

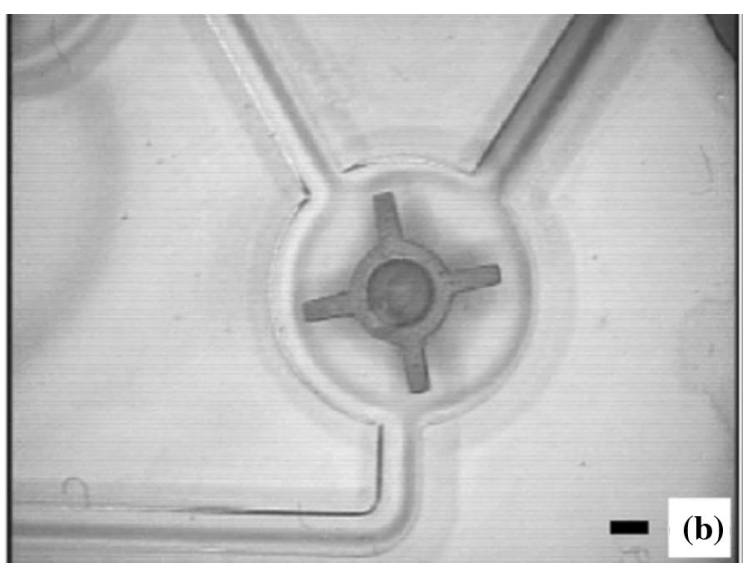

rotate freely. b In-flow of cold water causes the hydrogel ring to expand, thereby stopping the rotor's movement. From [131], (C2005 IEEE. Reproduced with permission 
temperature co-fired ceramic (LTCC) chip, and swelling and collapse of the gel structure are remotely controlled by an alternating magnetic field (AMF). Application of the AMF heats the $\mathrm{Fe}_{3} \mathrm{O}_{4}$ nanoparticles by Neel and Brownian relaxation, which in turn heats the hydrogel. Once the temperature exceeds the LCTS, the hydrogel collapses and opens the channel. Although the reported response time of the valve is in the order of minutes, which is unsuitable for microfluidics, the material itself may have potential for future approaches, since it facilitates spatially confined heating of complex hydrogel structures. Additionally, the response characteristics of the composite gel may be improved by reducing the dimensions and optimizing the material composition. Progress has recently been made in the understanding of the magneto-mechanical coupling of magnetic gels [138]. These gels feature well-defined coupling, magnetic nanoparticles that form the nodes of the polymer network, and torques on the particles that are directly transmitted to the connected polymer strands. In addition, it has been shown that by employing spindle-like hematite particles as exclusive cross-linkers in poly(acrylamide) gels, a new class of geometrically and magnetically anisotropic ferrohydrogels can be created [139].

\subsection{Electroactive PHs}

Electroactive hydrogels have anionic properties which cause positively charged surfactant molecules to bind to their surface. This surfactant layer of loosely bound molecules induces osmotic pressure between the interior and the exterior of the hydrogel, which results in bending of the gel structure. Kwon et al. developed an electroactive, paddle-shaped hydrogel actuator based on 4-hydroxybutyl acrylate (4-HBA) that has very low operating voltages $(<1.2 \mathrm{~V})$, low energy consumption, very short response times (exceeding a $5-\mathrm{Hz}$ oscillation frequency), and high durability. The actuator was used to realize a particlesorting device for cell culture media [140] and a valveless micropump [141] (Fig. 21). In [142] the familiar configuration of a cylindrical gel jacket based on 2-hydroxyethyl methacrylate (2-HEMA) around a supporting post was chosen for electroactive stimulation, with electrodes integrated in the bottom of the microchannel and buried in a dielectric parylene-N layer to enable electrolysis-free actuation. Use of the device was suggested for microfluidic flow regulation and-aligned at several positions alongside a microchannel and operated with a cascading electrical signal—for peristaltic pumping. Although they are not yet widely used in microfluidics, low operation voltages (i.e., low energy consumption) and biocompatibility make electroactive PHs particularly interesting for portable devices in the biomedical field. A method for effective improvement of the actuation response of a graphene hydrogel composite has been reported [143]: By means of a reshaping strategy consisting of loading in water, mechanical compression, and subsequent solidification, a (a)

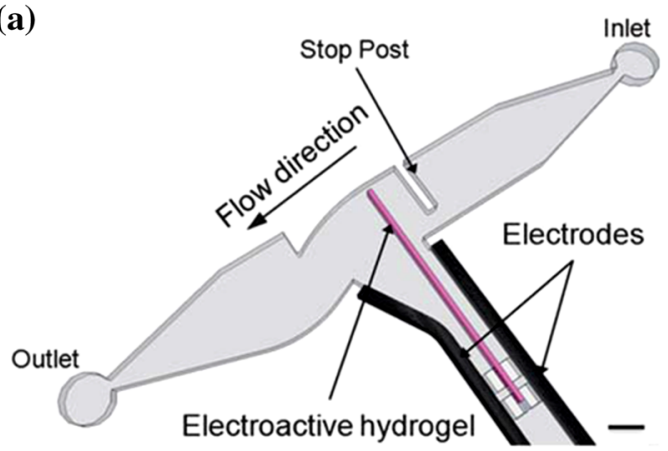

(b)

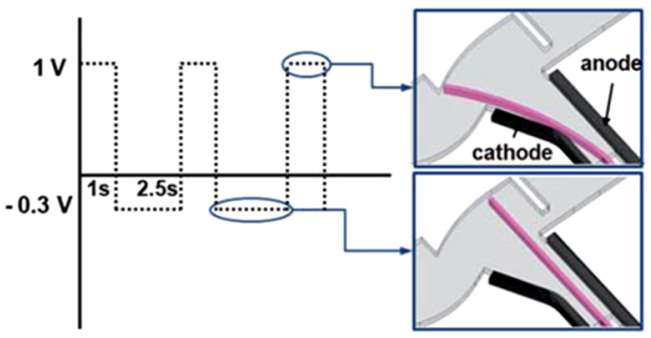

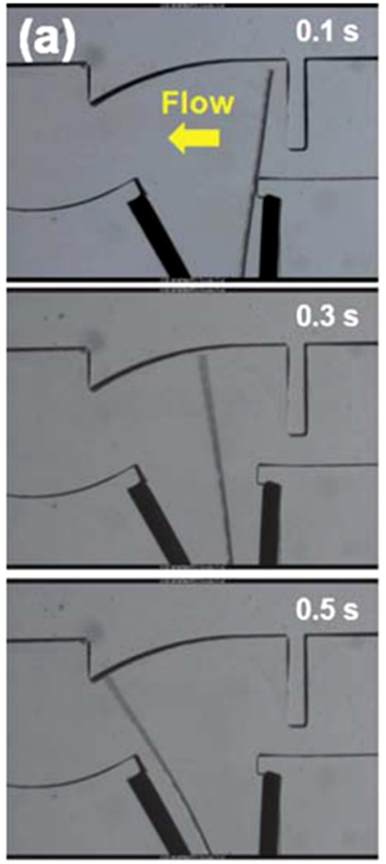

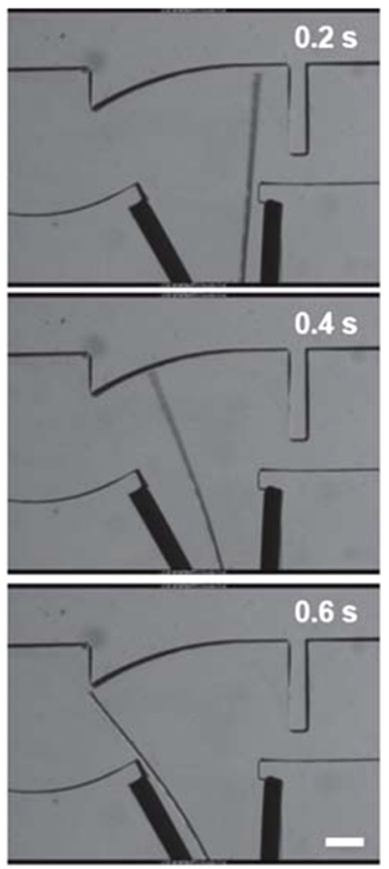

bending toward the anode. (Right) Photographs of the pumping sequence at $1 \mathrm{~V}$. From [141], (92011 The Royal Society of Chemistry. Reproduced with permission 

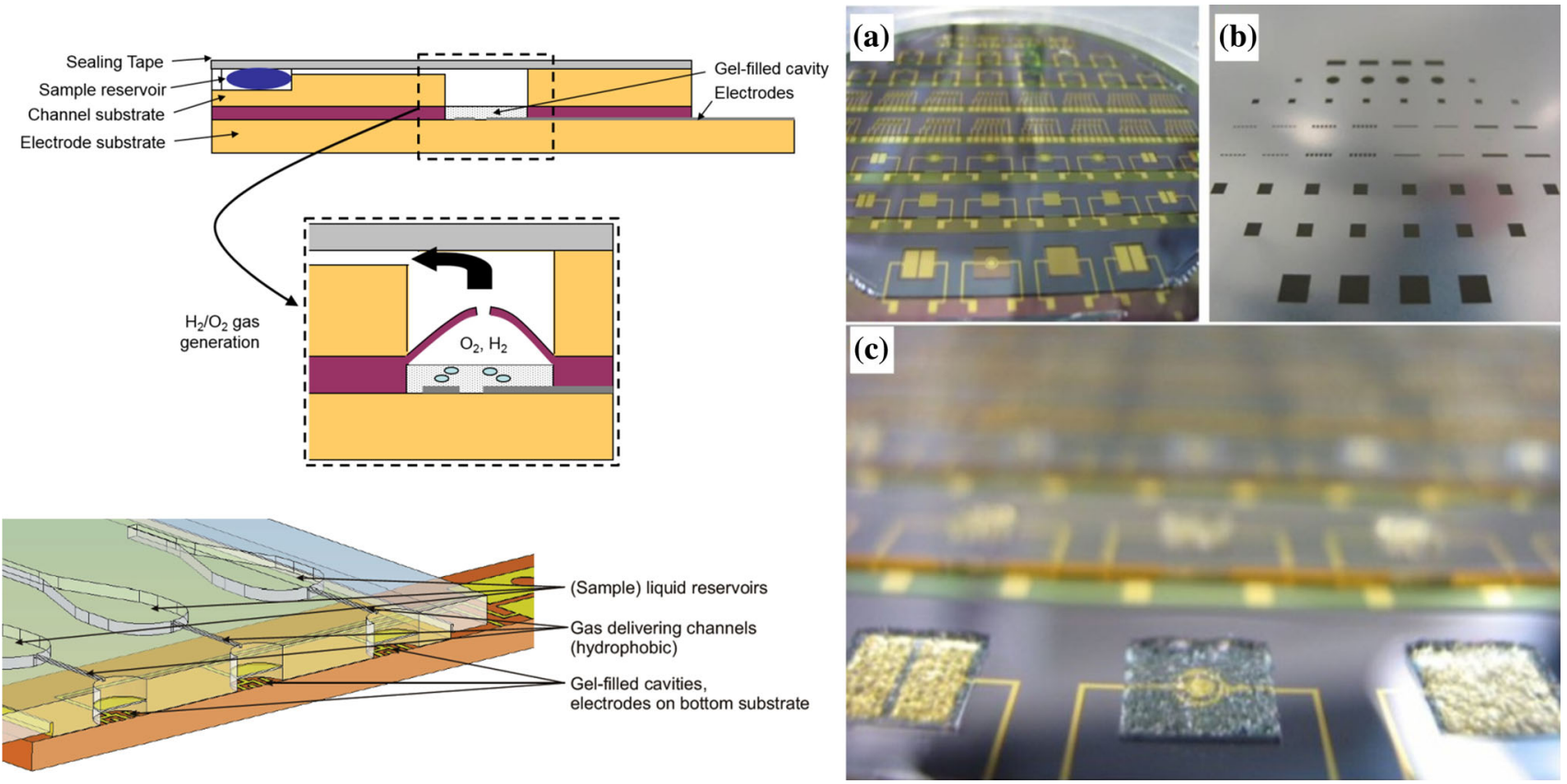

Fig. 22 (Left) Schematic drawing of electrolysis driven gel pump integrated in a LOC system. (Right) Filling of an electrolysis chamber for gas generation with hydrogel by using stencil printing: a four-inch silicon wafer with electrode test structures and SU8 cavities for the hydrogel, $\mathbf{b}$ stainless steel stencil, and $\mathbf{c}$ gel filled into cavities by using the stencil on a conventional screen printer. From [144], (C2010 Springer. Reproduced with permission

poly(acrylic acid) (PAA)-based hydrogel particles, which causes significant particle volume expansion and thus pushes fluid from an adjacent fluid reservoir at a predicted flow rate. Flow rates in the order of a few microliters per second and milligrams of the PAA particles and response times of a few minutes are achieved. A drug delivery system was demonstrated that uses the same volume displacement actuation scheme, but with a flat geometry designed to be attachable to skin [147]. An opposite approach, where the absorbent nature of dried hydrogel rather than its swelling in response to water is used for fluid actuation, was taken in [148]: The hydrogel facing the ambient acts as an evaporation pump which induces negative pressure in the connected microchannel. Although the concept of an evaporation pump is not new and the flow rates achieved were small (i.e., in the order of picoliters per second), the concept may be interesting for disposable and low-performance devices which require steady and long-lasting actuation. Additionally, the performance may be improved by integration of microheaters underneath the gel chamber. Using monodisperse latex nanoparticles as a sacrificial colloidal template, Kuroki et al. [149] prepared nanoporous thin-film membranes with a pore geometry that is tunable in response to specific water/solvent stimuli. The concept was proposed for applications such as flow control, size-selective filtration, and chemical and biological separation.

The same pumping principle as above, but exploiting the fundamental swelling properties of the hydrogels in response to water, was used for the approach in $[145,146]$. The pump is activated by adding water to 


\subsection{Self-oscillating PHs}

The driving force of a typical self-oscillating polymer (SOP) gel is the Belousov-Zhabotinsky (BZ) reaction, which is a non-equilibrium chemical reaction that generates autonomous oscillations of the redox-potential in the polymer. The common feature of these custom-made polymers is the presence of acid and covalently bonded ruthenium tris $\left(2,2^{\prime}\right.$ bipyridine) $\left(\mathrm{Ru}(\mathrm{bpy})_{3}\right)$ groups, which catalyze the $\mathrm{BZ}$ reaction. Due to this chemical oscillator, the polymer chains undergo spontaneous soluble-insoluble or, in the case of hydrogels, swelling-deswelling transitions. For example, a copolymer of the temperature-sensitive PNIPAAm hydrogel, $\mathrm{Ru}(\mathrm{bpy})_{3}$ and 2-acrylamino-2-methylpropanesulfonic acid (AMPS) was employed to induce a travelling chemical wave on the copolymer's surface, and the resulting autonomous peristaltic motion of the surface was successfully used for mass transport [150]. Maedea et al. [151] used the same copolymer composition to realize an SOP actuator that executes a pendulum motion. This was achieved by fixing one end of the cilia-like actuator. Furthermore, it was demonstrated that the period and amplitude of the gel actuator can be controlled by changing the composition, temperature and size of its gel structure. The maximum amplitude achieved by this actuator was in the range of millimeters and thus 100 times larger than that of conventional self-oscillating gel systems. The same research group developed a built-in system where all of the non-biorelated organic substrates of the BZ reaction were incorporated into the polymer chain [152]. The quarternary copolymer-including the metal catalyst, the $\mathrm{pH}$ control, and the oxidant-supplying sites-was designed to switch into self-oscillating mode only if the biorelated organic acids are present; promising applications in the context of biomaterials may therefore be expected. In [153], the authors focused on groundwork for the development of chemo-mechanically active systems by combining microfluidics for the supply of reactant solutions and SOP structures inside the microchannel. They showed that a continuous flow of the reactant solution led to by far the largest volume changes - in the order of $500 \%$-in BZdriven gel structures. A review of self-oscillating polymer systems driven by the BZ reaction was published by Zhou et al. [154]. They concentrated particularly on emerging techniques for the online study of self-oscillating behaviors and on engineering approaches to creating new types of SOP structures with unique functionalities.

\section{Liquid crystal elastomers}

Liquid crystal elastomers (LCEs) are composed of crystal molecules, so-called mesogens, and a compliant polymer backbone. The soft polymer backbone allows the mesogens to reorient between different phases, the mesophases, while preventing free flow of the molecules. The most prominent mesophases are (1) the isotropic phase, typically at high temperatures and characterized by random orientation of the molecules, and (2) the nematic phase, characterized by a long-range order of the molecules with their long axes roughly aligned. Reorientation of the mesogens between these phases induced by temperature changes or electric fields exerts stress on the polymer backbone, which in turn leads to bulk strain on the LCE. Due to heat diffusion, thermally actuated LCEs are rather slow (response time of $\sim 10 \mathrm{~s}$ ) and require active cooling, while electrically actuated LCEs, whose mesogens are intrinsically polarized, have short response times for moderate actuation fields in the order of a few $\mu \mathrm{V} / \mathrm{m}$, but also much smaller strains $(\sim 4 \%)$ than thermally actuated LCEs $(\sim 40 \%)$. In [155], recent developments in the field of LCE materials were reviewed with a focus on their use in actuator applications. Embedding nanoparticles in an LCE matrix is a promising strategy for increasing both functionality and performance of LCE-based actuators [156]. By selecting appropriate particles, the possible actuation mechanisms can be tuned for better and faster control and extended to include magnetic fields and broad-spectrum light. A review of cutting-edge programmable and adaptive mechanics realized by means of liquid crystal polymer networks and elastomers, together with their historical development, can be found in [157].

\subsection{Light-active LCE}

Van Oosten et al. [158] used an inkjet-printing process in combination with the self-assembling properties of a liquid crystal polymer to fabricate artificial cilia that are driven by light. Using multiple inks, they created microactuators with different subunits, which are then selectively addressed by changing the wavelength of the actuating light. The actuators can mimic the movement of natural cilia and have therefore the potential to be used for flow and mixing in microfluidic environments. In [159], the combination of an elastic pump membrane and a photodeformable LCE film, working against a fixed lid to induce pressure strokes on the flexible membrane and thus the pump chamber, was suggested as a micropump actuator and investigated both theoretically and experimentally. A robust strategy for in situ crafting of photodeformable graphene/LCE nanocomposites with superior and tunable actuation properties upon NIR irradiation has recently been reported [160]: The LCE composite was fabricated by in situ UV polymerization of liquid crystal monomers in the presence of graphene and concurrent hot stretching. 


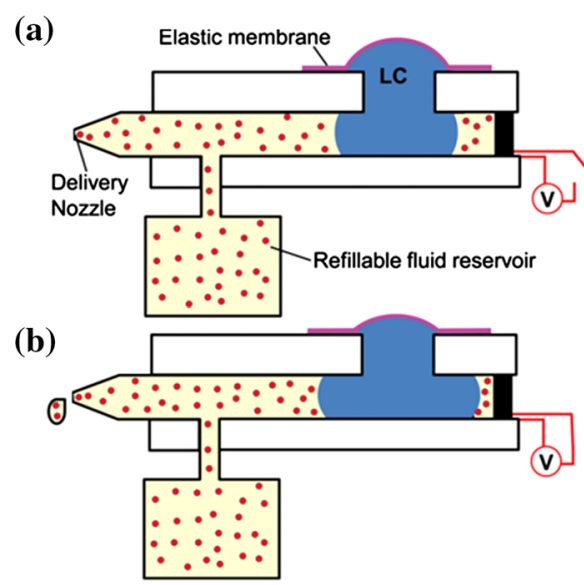

Fig. 23 System for continuous liquid delivery based on liquid crystal elastomers. (Left) Working principle: a LC droplet in contracted state and $\mathbf{b}$ LC droplet in stretched state, liquid is dispensed. (Right) Shape

\subsection{Temperature active LCE}

In [161], the temperature-induced transition of an LCE from the nematic to the isotropic state and back was used to cause abrupt buckling of the actuator, which sealed the microchannel. When they designed the valve, the authors considered the expansion of the LCE perpendicular to the director (initial orientation of the mesogens) and the shrinkage parallel to the director. In [162], a microfluidic double emulsion process was applied to fabricate LCE core-shell particles for use as one-piece micropumps. The LCE shell, at room temperature in the nematic state, contains a liquid core, which is reversibly pumped into and out of the particle by actuation of the liquid crystalline shell in a jellyfish-like motion. Although the presented particles were limited to temperature-sensitive LCEs, the fabrication method may be adopted to achieve responsiveness to light by incorporating azobenzene moieties or to electromagnetic fields by incorporating metallic nanopowder for localized plasmonic or inductive heating.

\subsection{Electric-field-active LCE}

In [163], the authors coated a millimeter-sized stripe of LCE in the nematic state with the electrically conductive polymer poly(3,4-ethylenedioxythiophene):poly(styrenesulfonate) (PEDOT:PSS) for Joule heating of the actuator. This configuration resulted in thermally induced elongation and compression of the LCE structure in the order of $30 \%$, and was used for the fabrication of bending actuators. Ren et al. [164] presented a dielectrically actuated LCE pump. In their approach, a small pillar-like droplet of LCE inside a cylindrical hole is in partial contact with embedded interdigitated electrodes. Application of voltage generates dielectric force that stretches the LCE droplet in change of the LC droplet dome under application of voltage. From [164], C2013 The Royal Society of Chemistry. Reproduced with permission

the direction of the electrode, which in turn exerts pressure on a neighboring fluid volume. The authors suggested, since the LCE droplet reverts to its initial state when the voltage is removed, the concept may be adapted-in a reciprocating manner-for use as a micropump (Fig. 23).

\section{Electrostrictive ferroelectric polymers}

Piezoelectricity in polymers can be achieved in three ways: by using bulk piezoelectric polymers such as poly(vinylidenefluoride) (PVDF) and its copolymers, by fabricating a piezoelectric polymer composite consisting of a polymer material with embedded inorganic piezoelectric material, or by using voided and charged polymers, also known as ferroelectrets or piezoelectrets [165]. Ramadan et al. [166] provided a comprehensive review of piezoelectric polymers as functional materials for electromechanical transducers, and compared them with common inorganic piezoelectric materials. However, so far only PVDF and its popular copolymer poly(vinylidenefluoride-co-trifluoroethylene) (PVDF-TrFE) have been used in microfluidics. In 2005, a PVDF-TrFE-based micropump diaphragm with a maximum displacement of $21 \mu \mathrm{m}$ in relatively high actuating electric fields of $106 \mathrm{~V} / \mu \mathrm{m}$ was presented [167]. In order to increase the response, a stack of PVDF-TrFE was later used as an electromechanical actuator for a nozzle-diffuser-type micropump [168]. This type of actuator achieved an actuation deflection of $80 \mu \mathrm{m}$ when an electric field of $90 \mathrm{~V} / \mu \mathrm{m}$ was applied. An innovative approach for the realization of PVDF-based actuators was presented by Pabst et al. [169]. They developed all-inkjetprinted PVDF-TrFE piezoelectric actuators on polyethylene terephthalate (PET) substrates with electrodes 

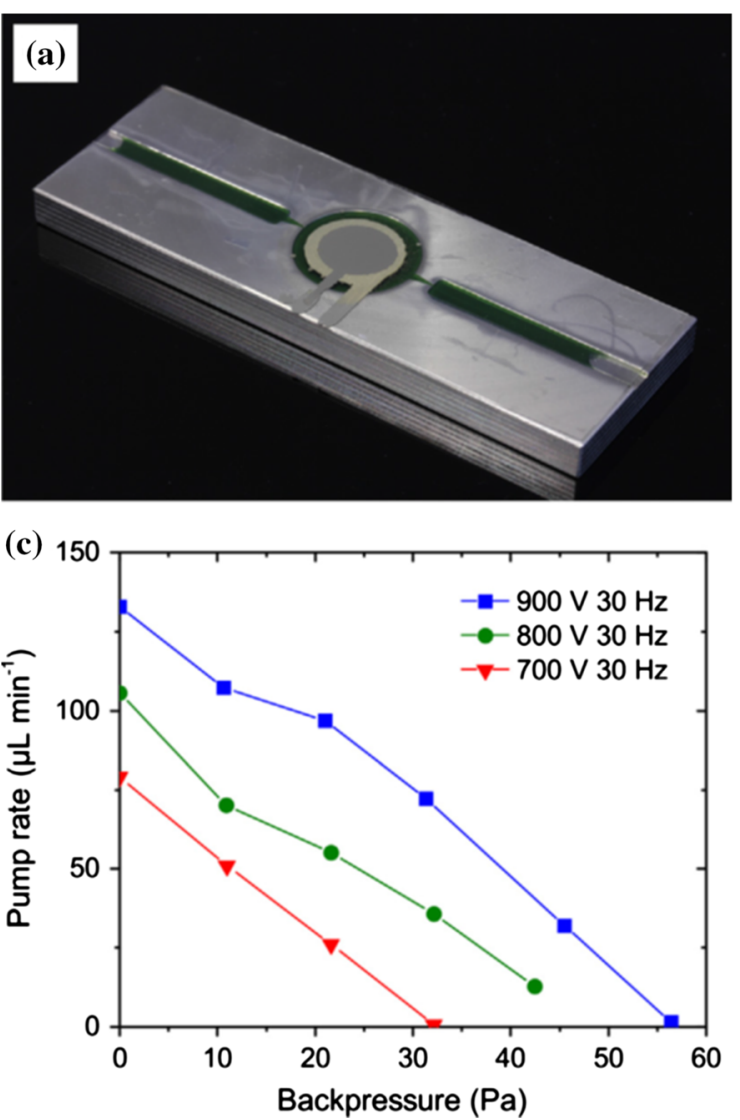

Fig. 24 Nozzle-diffuser-type pump demonstrator with an inkjetprinted $\mathrm{P}(\mathrm{VDF}-\mathrm{TrFE})$ membrane actuator (a, b). Pump rate with respect to backpressure (c) and frequency dependent pump rate (d).

printed from silver nanoparticle dispersions for low-cost membrane pumps. They found that the piezoelectric coefficients of the inkjet-printed PVDF-TrFE layers were in the same order as the values for the bulk polymer, and they successfully demonstrated the application of their inkjetprinted PVDF-TrFE actuator for microfluidic pumping in a nozzle-diffuser setup [170] (Fig. 24). Multilayer stack structures composed of alternating piezoelectric and polymeric elastomer layers have recently been proposed for use as active elements with specifically designed actuation characteristics and with overall improved actuation properties compared to the individual constituents [171]. For example, varying the thickness of the polymer stacks results in films which partly expand and partly shrink in response to an applied electric field and which can be used, for instance, to develop microfluidic gating architectures or caging geometries. Layered structures of PVDF with photoconductive material (titanium oxide phthalocyanine) serving as one of the electrode layers have been used to realize light-activated opto-piezoelectric thin-film actuators for microfluidic applications [172]. Independent actuation of multiple locations of the opto-piezoelectric film with a driving voltage source and a programmable light mask
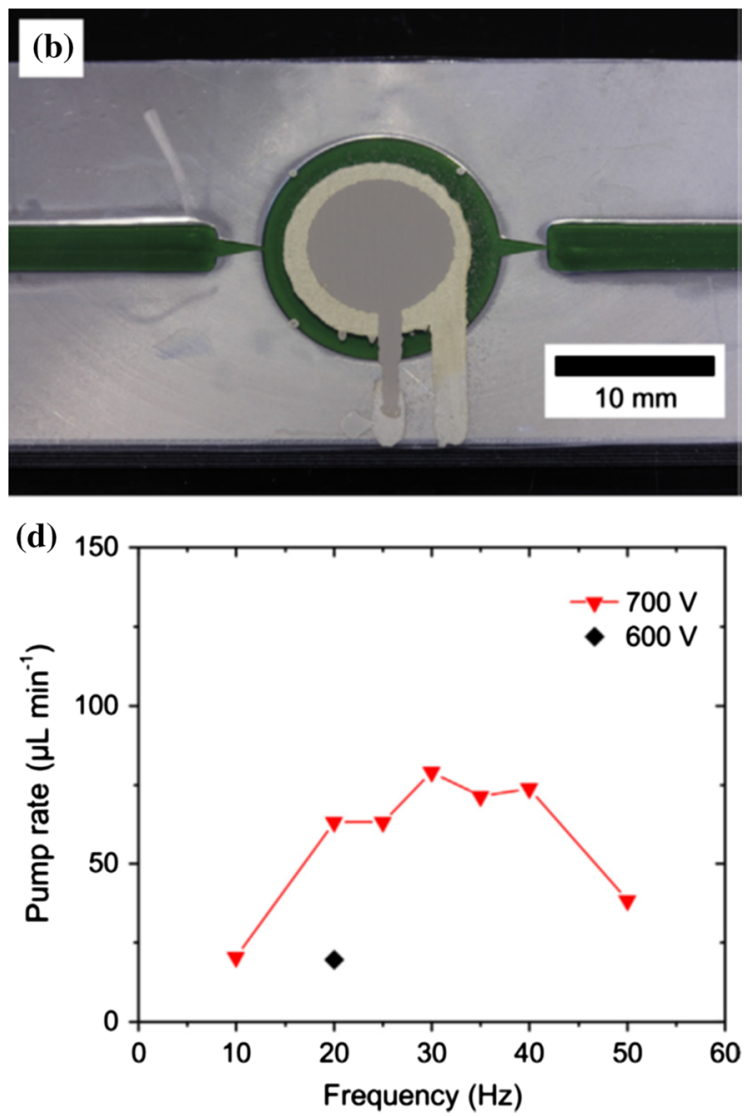

For quasi-static driving, pump rates up to $130 \mu \mathrm{l} / \mathrm{min}$ were obtained. From [170], (C2014 Elsevier. Reproduced with permission

allows complex microfluidic actuation schemes to be realized, as demonstrated by the authors in the form of light-mask-controlled micropumps. An innovative approach to improving the ferroelectret performance simply by blending with a low-cost plasticizer has been reported [173]. The material, a PVDF-based terpolymer (PVDF-TrFE-CFE) filled with a bis(2-ethylhexyl)phthalate (DEHP), exhibited a thickness strain $S_{33}=1.8 \%$ when an electric field of $10 \mathrm{~V} / \mu \mathrm{m}$ was applied, and its use as an active membrane in a nozzle-diffuser-type micropump was demonstrated. With this simple device, the authors achieved flow rates as high as $8 \mu \mathrm{l} / \mathrm{min}$ with $250 \mathrm{~Pa}$ backpressure for electric fields of only $15 \mathrm{~V} / \mu \mathrm{m}$ at $1 \mathrm{~Hz}$.

\section{Ionic electromechanically active polymers}

Ionic electromechanically active polymers (IEAPs) respond to a low-voltage stimulus $(<5 \mathrm{~V})$ by mechanical bending or buckling. The typical configuration of an IEAP bending actuator is a soft, porous polymer film whose mobile ions can move in response to an electric field and which is coated with two compliant electrodes [174]. 


\subsection{Ionic polymer metal composites}

A polymeric electrolyte of the IEAP sandwiched between two thin metal layers is called an ionic polymer metal composite (IPMC). Applying relatively low activation voltage (a few volts for samples with a typical thickness well below $1 \mathrm{~mm}$ ) leads to ion migration and redistribution due to the electric field, which in turn attracts water molecules to one side of the actuator stripe. Since nonuniform distribution of water in the polymer leads to swelling on one side and contraction on the other, the actuator bends. Using structured asymmetrical electrodes allows all kinds of geometrical deformations (e.g., twisting and twirling) to be realized. Almost simultaneously two reviews have recently been published $[175,176]$ that deal with the application of IPMCs for smart devices and focus on fabrication technology, theoretical modeling and control issues of IPMC-based devices. Most of the applications reported are in the field of biomimetic robots (especially swimming robots) and as sensor devices. Only a few applications in microfluidics have been reported so far. In [177], an IPMC-based diaphragm actuator was used to devise a valveless micropump with low operation voltages and large stroke volumes. The authors also presented a comprehensive finite element analysis of the achievable stroke volumes in relation to electrode shape and pump chamber pressure. A multilayered process was used by the authors of [178] to fabricate a flap valve IPMC membrane micropump embedded in a PDMS structure. Their device achieved a maximum flow rate of $\sim 0.8 \mathrm{ml} / \mathrm{min}$ with an applied voltage of only $3 \mathrm{~V}$ at $3 \mathrm{~Hz}$ driving frequency (Fig. 25). An advancement of the membrane actuation concept was reported by [179], which-unlike the conventional concept with a single membrane clamped at all edges-uses several IPMS actuators clamped at a single edge to drive an elastic film membrane. Due to the elimination of edge constraints and the separation of the IPMC from the pumping fluid, the authors accomplished a significant increase in membrane deformation and operational durability. Nevertheless, the stable operation time achieved (35 $\mathrm{min}$ ) remains insufficient for successful application in microfluidics and constitutes the main limitation. Ciliabased actuation schemes have also been explored in the context of IPMCs; a proof of concept where artificial IPMC cilia produced localized flow pattern disruptions in a laminar flow regime was reported in 2015 [180]. On the materials side, efforts were made to improve response time and achievable strain: use of spray-coated silver nanoparticle electrodes resulted in an improvement in response time by a factor of $\sim 3$ [181], and a specifically devised ionic polymer composite showed improved actuation displacement by a factor of 2 and provided stable performance for up to 2200 actuation cycles [182]. Furthermore, first attempts have been made to explore material degradation effects [183], and 3D printing of ionic electromechanically active polymer structures has been successfully demonstrated [184].

\subsection{Bucky gel actuators}

Bucky gel actuators (BGAs) have electrodes that are composed of carbon nanotubes (CNTs), and the porous structure in between is made of an electrically insulating but ion-permeable polymer membrane that contains an ionic liquid [57]. The exact working mechanisms are still disputed, but roughly speaking the electric-field-induced transport of ions and the associated dimensional change in the carbon particles leads to asymmetrically distributed stresses and thus deformations of the laminate structures. Recently, Kruusamäe et al. [185] have published (a)

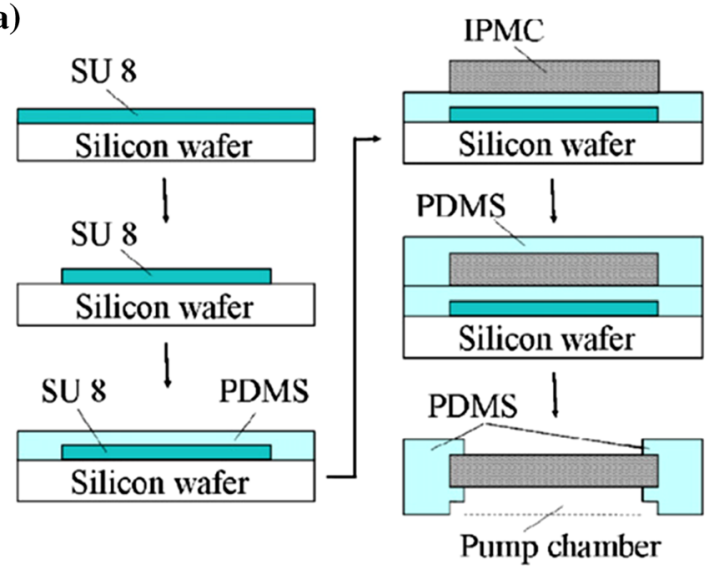

(b)

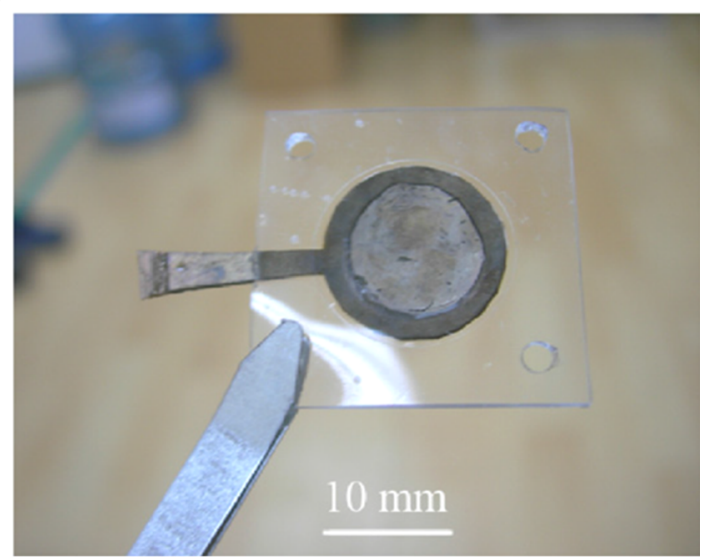

Fig. 25 Multilayer IPMC diaphragm actuator for pumping: a fabrication process and b photograph of the fabricated device (14 mm diameter and $0.6 \mathrm{~mm}$ thickness). From [178], (C)2008 Elsevier. Reproduced with permission 

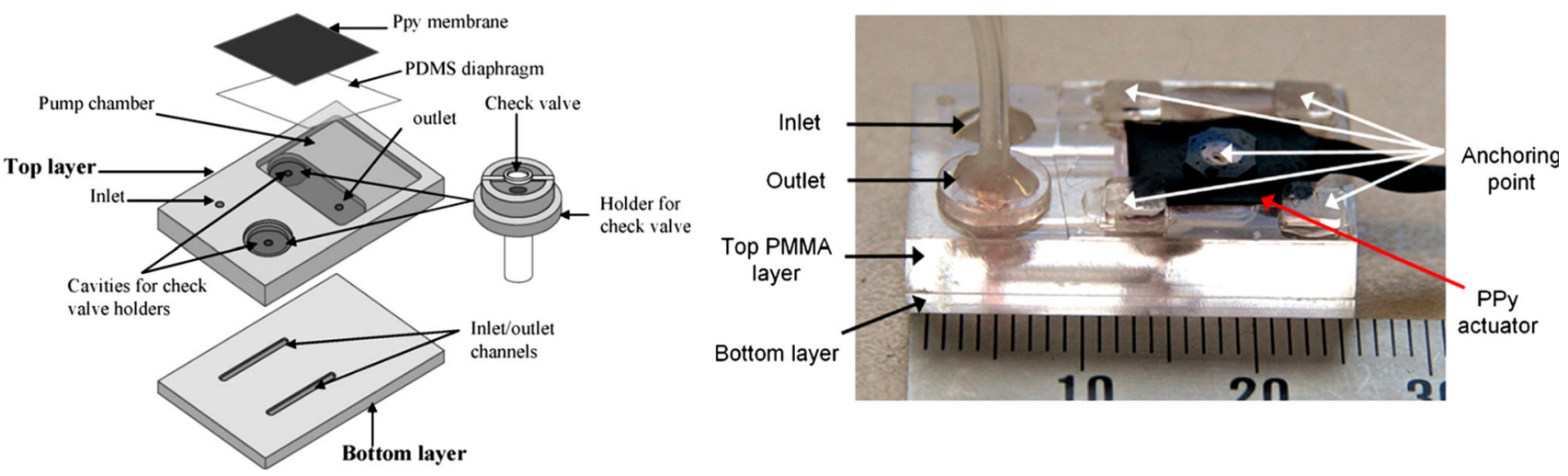

Fig. 26 (Left) Schematic drawing of the designed pump with passive check valves and PPy actuator. (Right) Photograph of the fabricated device. From [84], (C2008 Elsevier. Reproduced with permission

interesting work on the mechanical behavior of BGAs and the influence of their viscoelastic properties on actuation performance [186, 187]. In [188], the feasibility of using a BGA actuator as a microvalve in microfluidic point-of-care devices was demonstrated.

\section{Conjugated polymers}

Conjugated polymers (CPs), also referred to as conductive polymers, undergo considerable volume changes upon electrochemical oxidation or reduction. The most prominent representatives are polypyrrole (PPy), polyaniline (PANI), and poly(3,4-ethylenedioxythiophene) (PEDOT). Insertion or removal of ions requires the presence of an ion source or sink, respectively, which is either realized by an aqueous electrolyte surrounding the $\mathrm{CP}$ actuator or with a sandwiched polymer electrolyte and possibly with the whole device encapsulated to facilitate operation in ambient air or separated from the working fluid. Since the review by Jager et al. [189] of methods for microfabricating CP-based actuators and their potential future applications, various concepts for microfluidic actuation have been developed. In 2005, Wu et al. presented their TITAN concept, which stands for 'tube in tube actuator node' - a concentric arrangement of PPy actuators used to induce fluid movement through the inner channel. Sequential stimulation creates a peristaltic motion that allows fluid transport in a predefined direction, with a maximum flow rate of $2.5 \mu \mathrm{l} / \mathrm{min}$ at a square pulse operation voltage of only $1 \mathrm{~V}$ amplitude [190]. In [191], a somewhat related approach was presented for realizing a fluid pump where a capsule containing two squeezing PPy actuators capable of performing open and close movements and the liquid electrolyte were pulled over a standard silicone tube. By sequentially addressing the two actuators with driving voltages in the $1-\mathrm{V}$ range, peristaltic deformation of the tube was achieved, and fluid was pushed through the system. Additionally, the applicability of the concept for active transport of fluids with viscosities 400 times that of water was successfully demonstrated. Furthermore, membrane-type actuators based on PPy have been developed; in [84], a PPy actuator integrated into a PDMS structure above a fluid chamber in combination with two check valves was used to build a reciprocating micropump which achieved a maximum flow rate of $52 \mu \mathrm{l} / \mathrm{min}$ when operated at $\pm 1.5 \mathrm{~V}$ driving voltage (already mentioned in Sect. 3.3) (Fig. 26). The advanced membrane-type actuator concept described in Sect. 7.1 where membrane deformation is increased by using segmented actuators clamped at only one edge was in fact demonstrated first for PPy-based actuators. In [192], PPy petal actuators integrated into a flexible membrane for increased actuation performance were combined with flap check valves in a PDMS-based device to fabricate a reciprocating micropump with a maximum flow rate of $1.26 \mathrm{ml} / \mathrm{min}$ at an actuation voltage of $4 \mathrm{~V}$. Furthermore, a physics-based control-oriented model was developed to predict membrane deformation and flow rate in response to the electric field stimulation of the $\mathrm{CP}$ actuator.

Taccola et al. [193] have recently proposed using the hygroscopic nature of $\mathrm{CP}$ concurrently with its electrical conductivity for a new class of soft actuators. They discovered that $\mathrm{CP}$ films change their volume reversibly due to the absorption and desorption of water from vapor in air. Additionally, Joule heating induced by electric currents leads to volume contraction because of water desorption. They demonstrated that a bilayer of PDMS and PEDOT:PSS can be used as an actuator responsive to both active stimulation by electric signals and passive stimulation by changes in environmental humidity. Although the electrically controllable hygromorphic $\mathrm{CP}$ actuator was designed for operation in ambient air, a modified concept may also be of interest for microfluidic applications. 


\section{Phase-change actuators}

Typical phase-change actuators (PCAs) rely on the large volume expansion (10-15\%) associated with the solid-toliquid phase transition of selected materials-usually paraffin or other kinds of wax such as polyethylene glycol (PEG). The most common approach is to encapsulate a certain amount of paraffin wax in a stiff housing, to heat it either by integrated resistive heaters or from the outside via suspended particles and electromagnetic fields, and to direct the volume expansion toward a flexible membrane which deflects accordingly. Their simplicity and highpressure capability due to the low compressibility of paraffin make paraffin-based actuators particularly interesting, for example, for filter steps or pressure-driven separation in LOC devices [194, 195] (Fig. 27). Ogden et al. [196] have recently reviewed the historical background and the fundamental principles of paraffin actuation, and presented an overview of applications as actuators, valves and pumps. Although the vast majority of work in this field deals with paraffin as the working material, the actuation concepts can in principle be transferred to any other material (with or without phase transitions) that exhibits large volume expansion in response to temperature increase. For example, in [197] the temperature-sensitive fluid $3 \mathrm{M}$ Fluorinert FC40 (thermal expansion coefficient 0.0012 per ${ }^{\circ} \mathrm{C}$ ) was used to realize microfluidic valves based on thermally induced volume expansion. Other work addressed issues relating to the integration of PCAs (mainly valve structures) into microfluidic chips. Strategies for thermal isolation in miniaturized systems [198] and the large-scale integration of up to 588 thermally actuated phase-change valves for microfluidic applications [199] have been presented. Although the authors designed their device for the simplest case (they used the same material-tetradecane-as working fluid and as PCA material), they demonstrated a versatile platform for individual addressability of several hundreds of thermally actuated microvalves. This approach could potentially be applied to direct and indirect phasechange actuators, especially to paraffin-based ones, and to devices based on temperature-responsive hydrogels [123]. In [200], a paraffin-based valve suitable for use in a bendable lab-on-a-foil system is presented, together with an assessment of different actuator layer concepts for generation of large-volume strokes in layered modular systems. The major drawback of PCAs, the slow response time, may be overcome with the help of newly developed PCA composites, for example, compounds of paraffin and vertically aligned CNT films designed for large stroke actuators [201] or hybrids of PCA materials and conjugated polymers for fast thermomechanical actuation [202].

\section{Shape-memory polymers}

Shape-memory materials (SMMs) have the ability to recover their original shape after being quasi-plastically distorted in response to a particular stimulus such as heat, light or chemical substances. These materials therefore seem ideally suited to integrated microfluidics, as the material itself can sense and then generate a pre-programmed reactive motion. Shape-memory materials comprise the traditional shape-memory alloys (SMAs), shape-

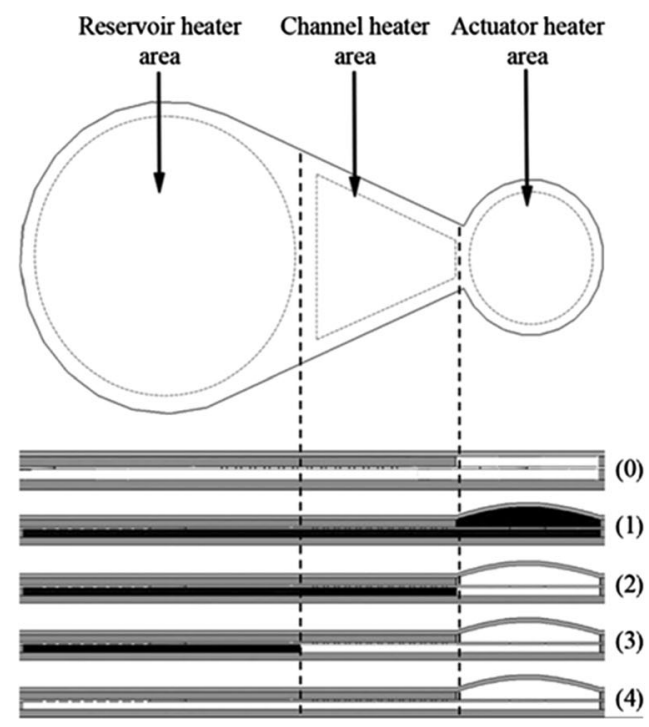

Fig. 27 (Left) Schematic cross-sectional view of the actuator principle (white solid paraffin, black liquid paraffin): $(0)$ all heaters off, (1) all heaters on, (2) actuator heater off, (3) channel heater off, (4)

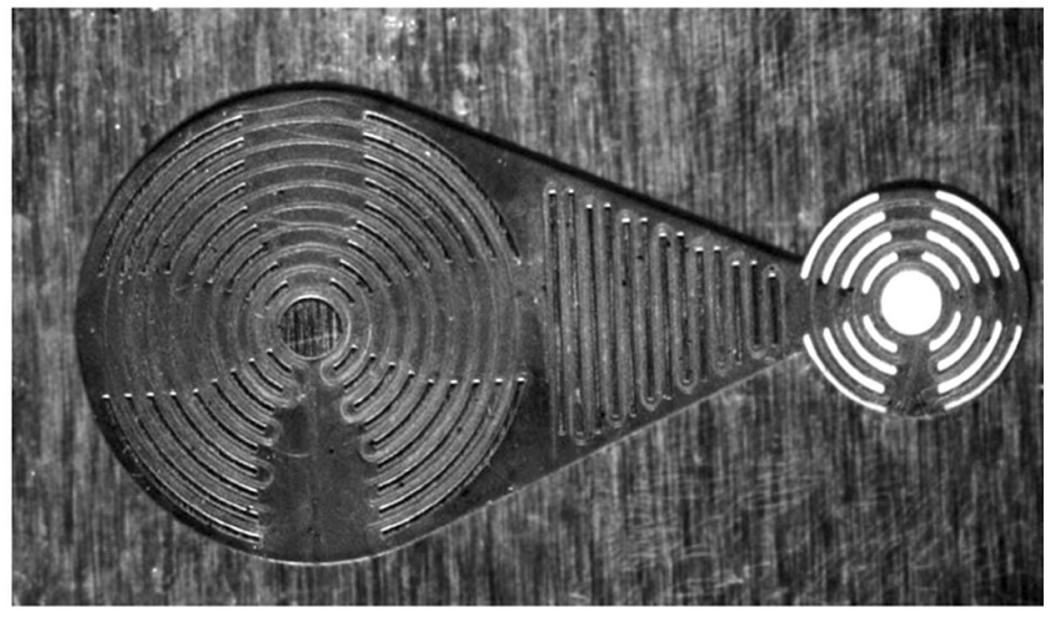

reservoir heater off. (Right) Photograph of the actuator. From [195], (C)2010 IEEE. Reproduced with permission 
memory polymers (SMPs), and the new shape-memory hybrids (SMHs), which seem to have great potential for microfluidic applications. The shape-memory effect of SMAs-typically made of $\mathrm{Cu}$-, NiTi- or Fe-based alloysis based on transitions between crystalline states (martensitic/austenitic), whereas for SMPs the glass transition from a hard to a soft phase is responsible for the shape-memory effect. SMHs are made of conventional materials which do not feature any memory effect individually, where one domain is always elastic while the other one (transition domain) is able to change its stiffness upon stimulation. Recently, Sun and coworkers [203] published a detailed review of stimulus-responsive SMMs and their applications as sensors and actuators. However, most of the applications reported so far can be assigned to the biomedical field for minimally invasive surgery, to the development of micro vehicles, deployable structures and morphing wings, and to systems for energy harvesting. Comparatively few applications as microfluidic transducers have so far been reported, and of these even fewer that use SMPs. One such rare example was described in [204], where a specifically devised SMP with a biologically relevant melting temperature was used to realize a microfluidic chip with dynamically tunable microchannel geometry. The authors studied the effects of dynamic changes in SMP channel geometry on the microfluidic flow, and complete channel closing was achieved by application of heat (Fig. 28). Most of the other reported applications in microfluidics used the betterknown SMAs, for instance, a NiTi-SMA microstructure as an active and self-regulating valve for microchannel cooling of next-generation photonic devices [205], SMA wires looped around elastomeric microchannels for electronic control of the flows inside the channels [206], and a configuration resembling a valve, where an integrated
SMA wire was used to stretch silicone tubing away from an inserted plug [207]. Though all of these concepts were realized with SMAs that are-at least in part—already commercially available, they can in principle also be fabricated by employing SMPs. Using SMPs rather than SMAs in microfluidics has several advantages [203]: For example, the raw material and processing costs are lower, the recoverable strain is typically an order of magnitude greater, the thermomechanical properties can be customized easily, they can be designed to be transparent, electrically conductive or magnetic, many of them are biocompatible and chemically stable, possible stimuli include heat, moisture, solvent or $\mathrm{pH}$ changes and light, and they can be actuated by more than one type of stimulus. Only recently has 3D printing of shape-memory polymers been demonstrated [208]. The printable resin was composed of a methacrylated semicrystalline polymer, a photoinitiator, a dye, and an inhibitor to prevent premature cross-linking and enabled the fabrication of SMP structures in almost any geometry; this raised its attractiveness for use in actuators significantly. In this context, also shapememory hybrids (SMHs) may become more attractive for microfluidic applications. In contrast to SMAs and SMPs, whose synthesis for specific applications usually requires extensive expertise in the chemical sciences, SMHs can easily be fabricated. A typical SMH configuration combines silicone as an elastic matrix with wax as a transition inclusion. In contrast to silicone, which keeps its elastic properties over a wide temperature range, wax becomes very soft when heated to its melting temperature. In this state, the composite can easily be compressed, and the elastic energy is stored in the silicone matrix. When cooled back to room temperature, the wax resolidifies, freezing the structure and thus preventing the release of elastic energy (a)

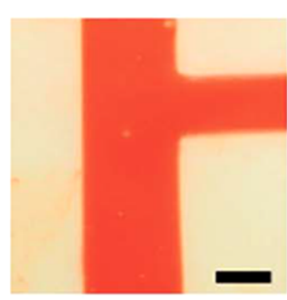

(b)

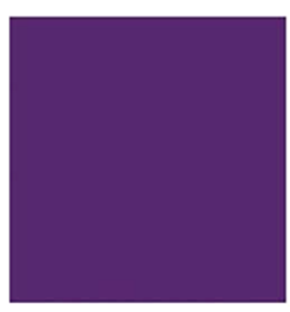

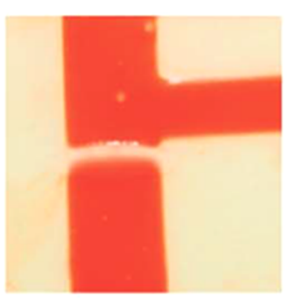

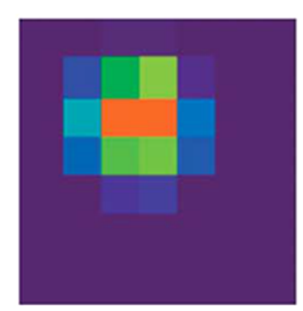

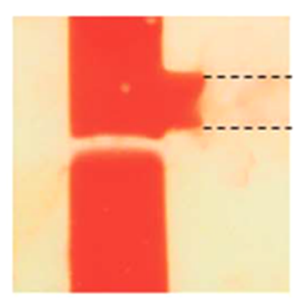

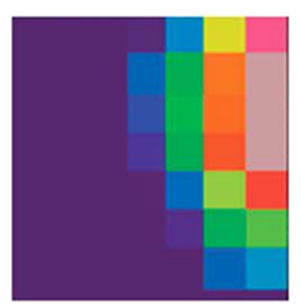

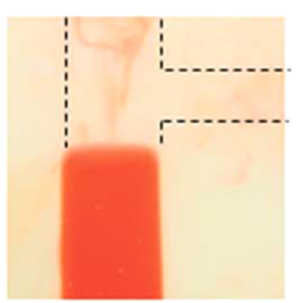
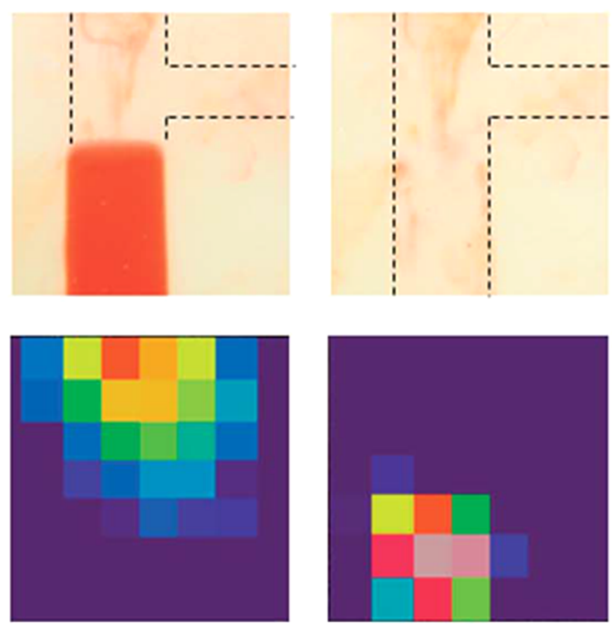

Fig. 28 a On-demand channel closing by local heating of SMP channels without valve. b Infrared images showing the points where heat $\left(\sim 40{ }^{\circ} \mathrm{C}\right)$ was applied. From [204], (C2013 The Royal Society of Chemistry. Reproduced with permission 


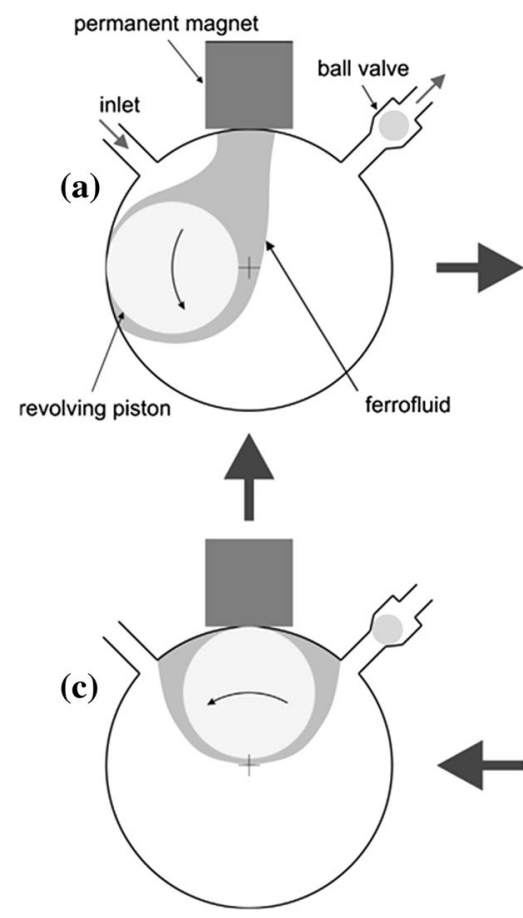

(b)
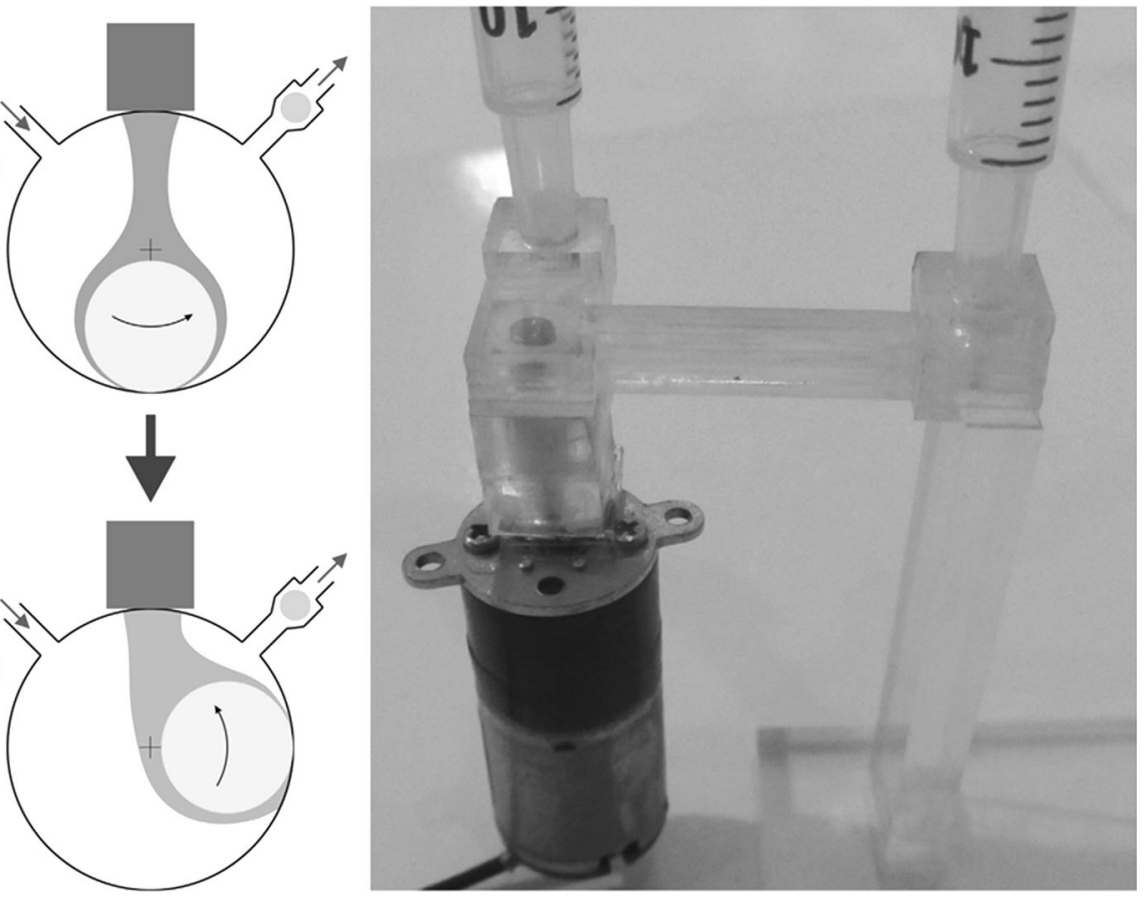

Fig. 29 (Left) Schematic illustration of pump principle, sequence a-d, using a revolving piston jacketed with ferrofluid. (Right) Photograph of micropump with external actuator. From [213], (C2015 Elsevier. Reproduced with permission

into the silicone matrix. Due to ease of fabrication and control, this type of composite will most probably gain attention for use in stimulus-active transducers in the near future.

\section{Magneto- and electrorheological fluids}

Magneto-rheological fluids Typically, magneto-rheological fluids (MRFs), also known as ferrofluids, are suspensions of synthetic oil and soft-magnetic nanoparticles. In response to an external magnetic field, the particles in the fluid align as long chains, which changes viscosity and flow behavior of the fluid considerably. This reversible change in physical properties stimulated by external magnetic fields can be used for actuators. In 2001, Zipser et al. [209] reviewed the behavior of MRFs in narrow channels in relation to variable magnetic fields and temperature. They showed that the viscosity, and thus the fluidic resistivity, of MRFs changes by more than an order of magnitude, and they discussed possibilities and limitations of using MRFs in smart actuators. In [210], ferrofluid plugs integrated into a glass-based microfluidic chip and actuated with external electromagnets were used to reproduce different types of valve functionality, and two valves and a pressure-generating piston were combined to operate as a pump. In their work, they also addressed the issue of water leakage around the ferrofluid plugs in untreated channels, which was significantly reduced by hydrophobic coating of the channels prior to assembly of the chip. In [211], two ferrofluid plugs within a circular microchannel and actuated with permanent magnets attached to a rotating stepper motor were used to realize a micropump with adjustable flow rate. A nozzle-diffuser structure connected to the ring served as passive in- and outlet valves and enabled a continuous pumping effect adjusted by the rotational speed of the stepper motor. The same actuation principle, a ferrofluid plug in a circular microchannel, was used by [212] as a valve and for fluid actuation on a whole-cell polymerase chain reaction (PCR) microchip. In [213], a revolving piston jacketed with ferrofluid in a rotary pump configuration accomplished both pumping and valving functionality (Fig. 29). A highly topical review by Yang et al. [214] surveyed recent applications of ferrofluids for microfluidic actuation.

Electrorheological fluids Similar to an MRF, an electrorheological fluid (ERF) is a type of colloidal suspension whose rheological properties can be changed by applying external electric fields. At field strengths in the order of $\mathrm{kV} /$ $\mathrm{mm}$, ERFs exhibit solid-like behavior, especially the ability to transmit shear stress. This transformation from liquid like to solid like is reversible and relatively fast, with response times in the order of $10 \mathrm{~ms}$. In [215], the authors used an ERF (consisting of urea-coated barium titanyl oxalate nanoparticles in silicone oil) with giant electrorheological properties (yield stress of $200 \mathrm{kPa}$ at an 
electric field of $5 \mathrm{kV} / \mathrm{mm}$ ) to realize microfluidic push-andpull valves in multilayer PDMS structures by sandwiching a PDMS membrane between an ERF channel and a microfluidic channel. The same research group realized an ERF-driven cross-stream-active micromixer in a PDMSbased device [216, 217], where the flow in the main channel is perturbed by the liquid flow in the orthogonal side channels. The device is driven by hydrodynamic pulsating pumps which consist of a chamber with a diaphragm and two out-of-phase ERF valves operating in push-pull mode. In [218], design and implementation of an ERF-actuated microfluidic pump with programmable digital control-also realized in multilayer PDMS technology-was presented. The authors also successfully incorporated ERF-based microactuators into a highly integrated microfluidic chip for DNA amplification [219] (Fig. 30). However, ERFs have so far been used mainly to realize microdroplet-based logic gates, which demonstrates the possibility of large-scale microfluidic processors for biological, chemical and computational experiments $[58,220]$. Recent publications [221, 222] provide a comprehensive review of the underlying physical mechanisms of ERFs and focus on applications for digitally controlled functionalities in microfluidics.
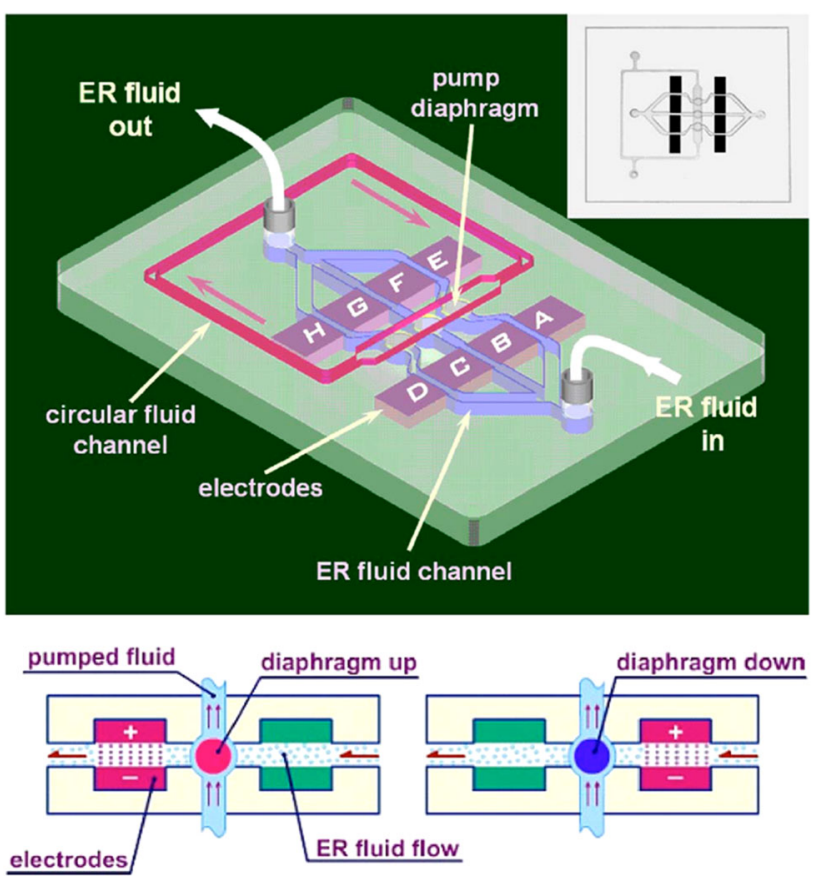

Fig. 30 (Top) Schematic illustration of ERF-driven micropump: the chip actuated by ER fluid controls the fluid circulation in the upper layer. The inset shows a picture of the fabricated device. (Bottom) ER valve operation principle showing the deformation of a single pump diaphragm. From [218], (C2006 AIP Publishing. Reproduced with permission

\section{Conclusions}

This review of actuation concepts for microfluidics based on stimulus-active polymers has shown that this field is surprisingly multifaceted and highly interdisciplinary with strong connections to materials science and polymer technology. This is simultaneously good news and bad news: On the one hand, there is no single type of actuator which would fit all possible applications in LOC or $\mu$ TAS solutions; on the other hand, the huge variety of polymer-based actuator concepts available — each with its own advantages and disadvantages-offers a 'toolkit' from which an approach to a particular application can be selected. In fact, the requirements for actuator concepts capable of being integrated into microfluidic chips are heavily applicationdependent and vary greatly. For example, some applications call for high-pressure actuation, others are designed for one-way use only. Thus, it is highly improbable to satisfy all requirements with a single actuation concept.

Quantitative comparison Table 1 provides a quantitative comparison of the different stimulus-responsive materials by the two main applications in microfluidics, namely as micropumps and microvalves. Generally, the achieved flow rate of the presented pump concepts varies from $\mathrm{nl} / \mathrm{min}$ to $\mathrm{ml} / \mathrm{min}$ with a concentration in the $\mu \mathrm{l} / \mathrm{min}$ range, thus covering the whole spectrum of flows occurring in microfluidic applications. However, the ability to generate pressure respectively to operate the pump against back pressure is much more heterogeneous for the different material classes. Phase-change actuators induce the highest reported pressures (up to $\sim 2 \mathrm{MPa}$ ), followed by concepts based polymer hydrogels, some membrane-based devices (e.g., DEA-actuated, PDMS composites and conjugated polymers), and magneto-rheological fluid based concepts which are well below in the order of 1-10 $\mathrm{kPa}$. Then, with pressure generation capabilities in the order of $0.1 \mathrm{kPa}$ and below emerge the PVDF-based devices, acoustic streaming with microstructures and cilia-based concepts, respectively. For microvalve applications again polymer hydrogels and phase-change actuators dominate the high-pressure regime, with maximum pressures in the range between $10 \mathrm{MPa}$ and $100 \mathrm{kPa}$ that can be applied to the valves, followed by some membrane-based concepts (e.g., bistable PDMS membranes and electrostatically actuated membranes) which feature maximum operating pressures slightly above or below $100 \mathrm{kPa}$. However, for microvalve application the switching time is a crucial parameter, and here phasechange actuators and polymer hydrogels have-featuring values from a few seconds to a few minutes-a clear handicap. The smallest switching times in the millisecond range were achieved with a bistable membrane configuration (thermopneumatically actuated), a PDMS membrane 


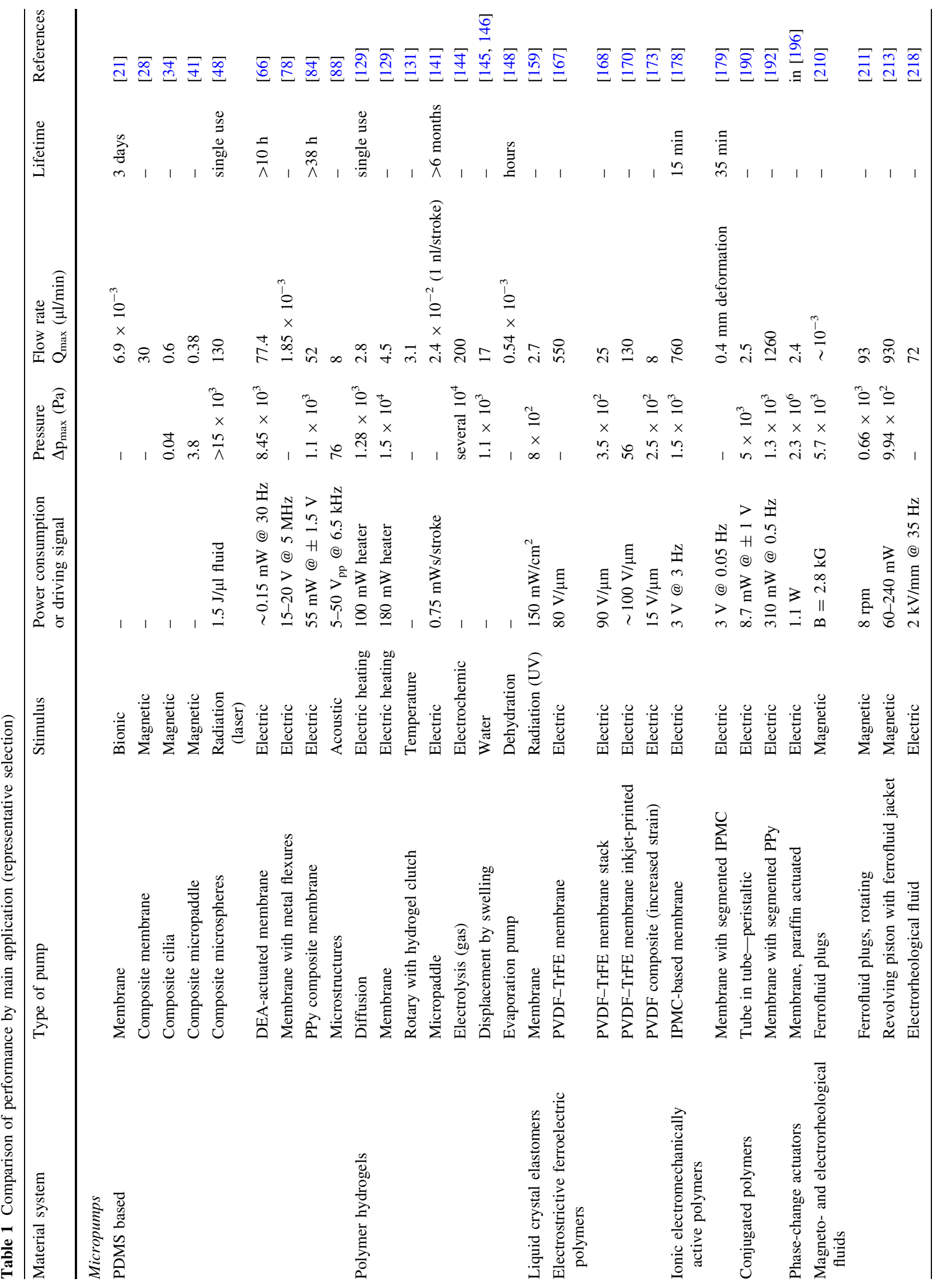




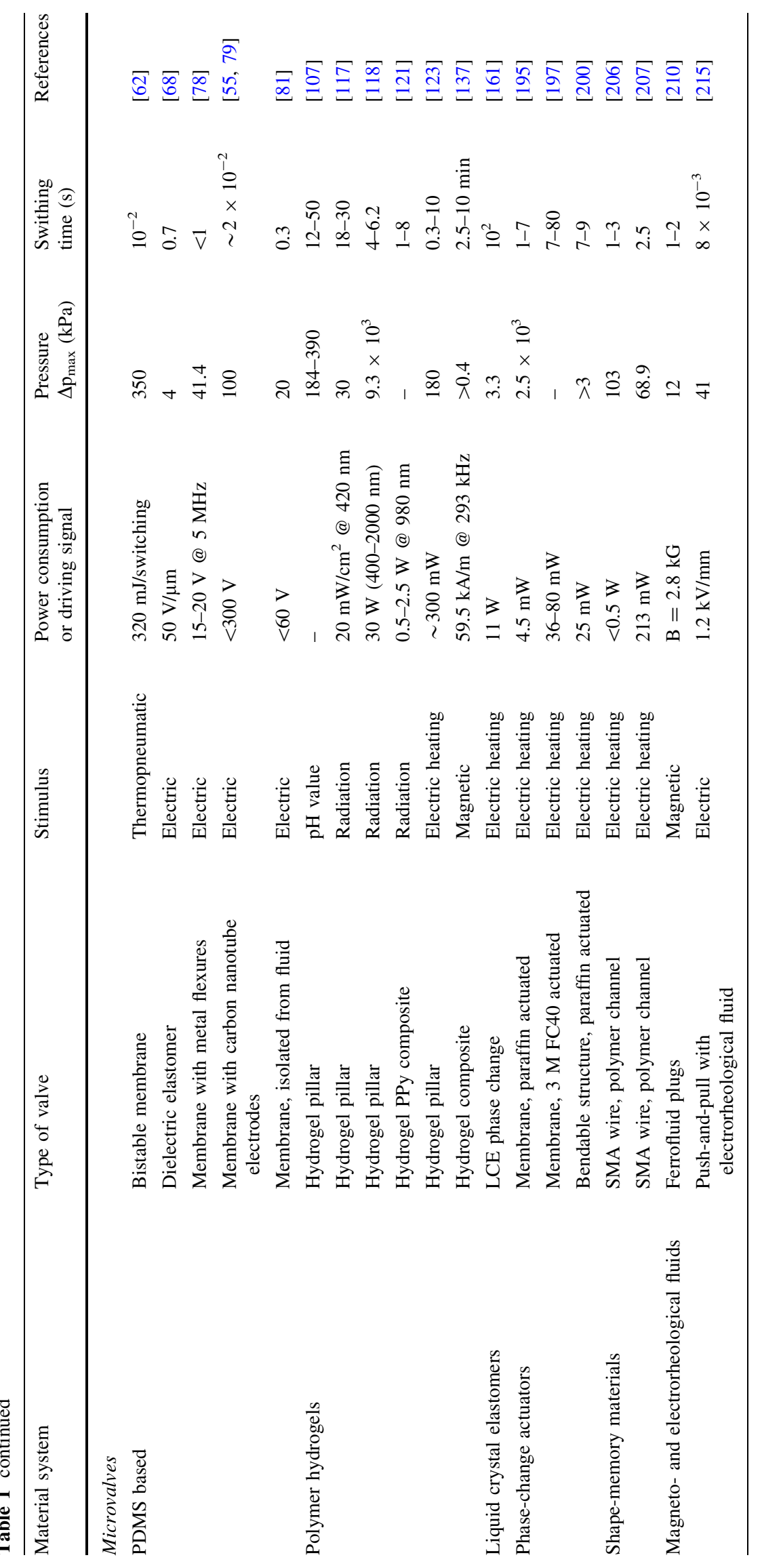


with carbon nanotube electrodes and electrostatic actuation, and with push-and-pull valves based on electrorheological fluids (cf. Table 1).

It is quite instructive to compare these values with the performance of the so-called 'Quake-valve', which-since its introduction in 2000 by Unger et al. [223] — has evolved to a kind of benchmark for microfluidic valves and pumps. They developed a multilayer soft lithography technique and used it to fabricate valves in a cross-channel architecture with a thin separating membrane. When pressure is applied to the upper channel, the 'control channel', the membrane deflects and closes the lower fluid channel. By sequentially addressing three valves aligned in a row above a fluid channel peristaltic pumping can be realized too. With $40 \mathrm{kPa}$ pressure difference between the control and fluid channel the valve was completely closed, featuring switching times in the millisecond range. The peristaltic pump achieved a flow rate of $0.14 \mu \mathrm{l} / \mathrm{min}$. None of the valves showed any signs of wear or fatigue, even after more than 4 million actuations. Except for the fact that the Quake-valve needs an external pressure source, the performance of the concept is impressive particularly with regard to its simplicity. However, a quick look at Table 1 shows that several actuators based on stimulus-active polymers feature comparable performance.

Lifetime issues, as far as examined, favor PDMS hybrid concepts (e.g., electrically or magnetically actuated membranes, dielectric elastomer actuators and shape-memory hybrids) and PVDF-based actuators. For example, over 400 million actuation cycles have been demonstrated for silicone-based DEAs [224], and their change in electromechanical properties for a time period of several months has been studied recently [225]. The suitability of PVDF and its most important copolymer PVDF-TrFE for low earth orbit applications has been demonstrated in [226], which consequently qualifies the material also for the use in microfluidic devices. And a hybrid concept of a shapememory alloy (SMA) actuator integrated into a PDMS chip showed stable operation for at least 10,000 cycles [206]. On the contrary, concepts involving the oxidation and reduction of the polymer matrix, such as in the case of conjugated polymers (CPs), or the diffusion of ions in ionic electromechanically active polymers (IEAPs) suffer from poor lifetime in the order of a few minutes to hours. So far, up to 2200 actuation cycles for an IPMC-based actuator have been demonstrated [182]. Polymer hydrogels are somewhere in between with reported lifetimes from several hours to more than 6 months.

The power consumed by the functional polymers is in general rather low ranging, with a few exceptions, from a few $\mathrm{mW}$ to a few hundred $\mathrm{mW}$. Hence, all of these concepts are well suited for mobile, battery-powered systems. Except for the two autonomously driven concepts, the use of biological cells (cf. Sect. 3.1) and self-oscillating polymer hydrogels (cf. Sect. 4.8), actuators based on functional polymers require some consideration about the way how to apply the stimulus. In general, concepts based on electric stimulation necessitate contact pads, whereas magnetically responsive materials and actuators sensitive to light irradiation/temperature can be remotely powered. The former group comprises all variants of electrostatically actuated PDMS membranes, polymer hydrogels (PHs), either electrically stimulated or electrically heated, most of the liquid crystal elastomers (LCEs), actually all concepts based on electrostrictive ferroelectric polymers (PVDF), ionic electromechanically active polymers (IEAPs) and conjugated polymers (CPs), as well as the-in the main-electrically heated phase-change actuators (PCAs) and shape-memory polymers (SMPs), and finally devices based on electrorheological fluids (ERFs). The latter group essentially covers any type of magnetic composite, straight through all material classes, and materials responsive to electromagnetic radiation, either in a direct manner such as in the case of LCEs (cf. Sect. 5.1) or PHs (cf. Sect. 4.2), or an indirect manner by using radiation instead of resistors for local heating.

Pros and cons at a glance PDMS as a material for realizing soft actuators still holds an exceptional position-at least in scientific research-mainly due to its remarkable transformation ability with respect to its physical and mechanical properties. It has evolved from a polymer for pure prototyping of microfluidic structures to a base material for the development of specifically devised functional materials. Thus, it is now difficult to imagine research into polymer-based actuators without PDMS. The material is extremely versatile: it can be made responsive to almost any conceivable physical stimulus when used in composites, layered structures, or in the form of microstructures. Polymer hydrogels (PHs) are fundamentally different from PDMS in that they are naturally responsive to almost any kind of stimulus; this is both their greatest advantage and disadvantage. PHs are ideally suited to application in fluidic environments, so their use in low-cost or disposable LOCs seems intuitive. Various strategies have been developed for better and faster actuation control to be integrated into advanced microfluidic structures. Some approaches aimed for better temperature control at small scales, which becomes possible with magnetic PH composites, heating by infrared light with masks, or the integration of electric microheaters. An attractive alternative for autonomous microfluidic chips are self-oscillating PHs, although robustness and lifetime of the actuation concept remain open issues. Liquid crystal elastomers (LCEs) play a relatively minor role in microfluidic actuation, but selected concepts may prove interesting for application in microfluidics. In particular, thermally responsive LCEs- 
either directly actuated by integrated microheaters or with admixed particles responsive to light or electromagnetic fields-exhibit adequate response times and considerable strains. Electrostrictive ferroelectric polymers are also rarely used in microfluidics, probably due to the rather small achievable strain and the relatively high actuation voltages. However, stacked geometries with enhanced actuation response and technological advances in processing PVDF and its copolymers make this class of materials increasingly interesting for microfluidic actuation, particularly for high-end applications that require precise motion control. The same applies in principle also to actuator concepts based on conjugated polymers (CPs), but with the additional advantage of remarkably low actuation voltages for comparable strains. Thus, CP-based actuators are promising candidates for integration into LOC and $\mu$ TAS devices. Ionic polymer metal composites (IPMCs) also have high strains at low operation voltage-their applicability in microfluidics has already been demonstrated-but their robustness and lifetime are at this point insufficient for application in LOC devices. The same applies to bucky gel actuators (BGAs) - this interesting physical concept requires considerable development on the materials side in order to become attractive for integration into microfluidic chips. Phase-change actuators (PCAs) inherently have slow response times, since the PCA material must undergo solidto-liquid phase transitions and vice versa, but the concept is ideal for high-pressure fluid actuation. However, progress has been made in reducing the response time of PCA materials either by using nanomaterials mixed with the phase-change material or by developing new materials with increased volume expansion coefficients. Shape-memory polymers (SMPs) can be used for high-pressure fluid actuation and are also ideal for microfluidics due to their inherent sensing capability and ability to execute preprogrammed movements. Promising recent developments concern the realization of hybrid shape-memory materials (SMHs), which combine the functionality of SMPs with the advantage of low production costs and ease of fabrication. Finally, electro- and magneto-rheological fluids (ERFs and MRFs) are particularly attractive for microfluidics, since response times are very short, the achievable deformations are large, and the exact position of actuation can be tuned.

Actuation and deformation modes Based on the literature on the application of functional polymers reviewed, it is obvious that certain actuation schemes-namely more or less equally membrane- and piston-based approaches for fluid actuation as well as for valving, and in second place concepts mimicking the behavior of natural cilia for pumping and mixing-are preferred for integration into microfluidic devices (cf. Fig. 3). The associated deformation modes are predominantly two-dimensional membrane deformations, three-dimensional volume expansions, and one-dimensional beam deflections, respectively (cf. Fig. 4). Only hydrogel-based actuators have been used to realize the whole set of deformation modes, all other material classes exhibit at least one gap in this scheme. Cilia-based concepts have been realized with a broad spectrum of materials, ranging from composites to polymer hydrogels and liquid crystal elastomers. Membrane-based transducers, on the other hand, have been realized with virtually any type of material, and thus represent the most popular and successful approach to fluid actuation with the help of functional polymers. Three-dimensional volume expansions and contractions have mainly been realized using polymer hydrogels, shape-memory polymers, and phasechange actuators.

Feedback control An issue frequently overlooked in the context of microfluidic actuation is the possibility of implementing some kind of in-line feedback control of the deformation mode. For some stimulus domains (e.g., electric and magnetic), feedback control can in principle be realized by using the actuation pathway itself, for instance, by measuring the electrical impedance or induced voltages due to changes in magnetic flux density. For other stimulus domains, specifically tailored concepts for in-line control must be developed. One possible strategy that has already been demonstrated is the dispersion of electrically conductive particles in the polymer matrix and measurement of the changes in electrical conductivity or resistance that accompany the deformation of the polymer actuator [49].

The use of composites A conspicuous feature of recently presented novel and innovative concepts for polymer-based actuators is the use of composites in various ways-in the form of layered or foamed structures, as homogeneous mixtures of two or even more constituents, and as suspensions of polymers with small particles that induce an additional functionality. The effects achieved include (1) improved actuator performance in terms of response time or actuation force/amplitude, (2) geometric composite structures for specifically tailored movements in response to external stimuli, (3) the realization of actuators that are responsive to multiple physical stimuli, and-more importantly-(4) the transformation of naturally unresponsive into responsive polymers. From a present-day perspective, based on the steadily increasing number of publications in this field, composite materials will play a major role in the development of highly integrated LOC solutions in the near future. Generally, multiresponsive materials are unsuited for transducers due to potential cross-sensitivities and spurious effects, but they open up the possibility of realizing complex actuation schemes. A good example is the combination of a photoconductive material with PVDF in phototriggered opto-piezoelectric actuators [172]. 
Performance issues The inherent drawbacks of small achievable strains (and thus actuation forces) and long response times in some polymer-based actuator concepts have been addressed in two different ways. (1) The chemical and/or compositional structure of the material itself was modified in order to increase the sensitivity to an external stimulus, and (2) specific constructional designs were developed in order to enhance or even multiply the response of the actuator. For example, bi-stability effects were exploited in minimum-energy structures, and stacked multilayer geometries were realized. With a few exceptions, only little attention has been paid to long-term stability (aging effects) and reproducibility of the presented actuation concepts, even though this can be considered a basic requirement for the commercial success of microfluidics. Outside the scientific community, polymers are still commonly associated with problematic characteristics such as low quality and poor durability.

Processing and technology An emerging trend concerns the processing of polymers and their composites. While casting, spin-coating and related methods derived from thin-film and microsystems technology have previously been the most common methods for realizing polymer structures, direct-writing and additive manufacturing methods are gaining in popularity. The increasing performance of $3 \mathrm{D}$ printing technologies in terms of resolution and spectrum of usable materials combined with high-resolution polymer jet- and laser-assisted manufacturing technologies opens up new and exciting possibilities for the design and realization of specifically devised actuator configurations without the need for time-consuming preparation of casting molds or soft lithographic process steps. Even complex composite structures can now be processed with direct-writing methods, which combine the advantage of enhanced functionality with moderate technological effort [227].

Acknowledgments The author wishes to express his deep gratitude professor Siegfried Bauer from the Soft Matter Physics Institute at the JKU Linz for his precious advices during the preparation process of the manuscript.

Open Access This article is distributed under the terms of the Creative Commons Attribution 4.0 International License (http://creative commons.org/licenses/by/4.0/), which permits unrestricted use, distribution, and reproduction in any medium, provided you give appropriate credit to the original author(s) and the source, provide a link to the Creative Commons license, and indicate if changes were made.

\section{References}

1. T.M. Squires, S.R. Quake, Microfluidics: fluid physics at the nanoliter scale. Rev. Mod. Phys. 77(3), 977 (2005)

2. J. Castillo-León, W.E. Svendsen (eds.), Lab-on-a-Chip Devices and Micro-Total Analysis Systems: A Practical Guide (Springer, New York, 2014)
3. L.R. Volpatti, A.K. Yetisen, Commercialization of microfluidic devices. Trends Biotechnol. 32(7), 347-350 (2014)

4. I.E. Araci, P. Brisk, Recent developments in microfluidic large scale integration. Curr. Opin. Biotechnol. 25, 60-68 (2014)

5. Y. Temiz, R.D. Lovchik, G.V. Kaigala, E. Delamarche, Lab-ona-chip devices: how to close and plug the lab? Microelectron. Eng. 132, 156-175 (2015)

6. P. Gravesen, J. Branebjerg, O.S. Jensen, Microfluidics-a review. J. Micromech. Microeng. 3(4), 168-182 (1993)

7. H. Becker, C. Gärtner, Polymer microfabrication technologies for microfluidic systems. Anal. Bioanal. Chem. 390(1), 89-111 (2008)

8. G. Spizz, L. Young, R. Yasmin, Z. Chen, T. Lee, D. Mahoney, T. Roswech, Rheonix CARD ${ }^{\circledR}$ technology: AN innovative and fully automated molecular diagnostic device. Point of Care 11(1), 42-51 (2012)

9. D.J. Laser, J.G. Santiago, A review of micropumps. J. Micromech. Microeng. 14(6), R35 (2004)

10. K.W. Oh, C.H. Ahn, A review of microvalves. J. Micromech. Microeng. 16(5), R13 (2006)

11. D. Dumont-Fillon, H. Tahriou, C. Conan, E. Chappel, Insulin micropump with embedded pressure sensors for failure detection and delivery of accurate monitoring. Micromachines 5(4), 1161-1172 (2014)

12. D.C. Duffy, J.C. McDonald, O.J. Schueller, G.M. Whitesides, Rapid prototyping of microfluidic systems in poly (dimethylsiloxane). Anal. Chem. 70(23), 4974-4984 (1998)

13. J.R. Anderson, D.T. Chiu, R.J. Jackman, O. Cherniavskaya, J.C. McDonald, H. Wu, S.H. Whitesides, G.M. Whitesides, Fabrication of topologically complex three-dimensional microfluidic systems in PDMS by rapid prototyping. Anal. Chem. 72(14), 3158-3164 (2000)

14. J.C. McDonald, G.M. Whitesides, Poly (dimethylsiloxane) as a material for fabricating microfluidic devices. Acc. Chem. Res. 35(7), 491-499 (2002)

15. F. Schneider, T. Fellner, J. Wilde, U. Wallrabe, Mechanical properties of silicones for MEMS. J. Micromech. Microeng. 18(6), 065008 (2008)

16. P. Jothimuthu, A. Carroll, A.A.S. Bhagat, G. Lin, J.E. Mark, I. Papautsky, Photodefinable PDMS thin films for microfabrication applications. J. Micromech. Microeng. 19(4), 045024 (2009)

17. D.P.J. Cotton, A. Popel, I.M. Graz, S.P. Lacour, Photopatterning the mechanical properties of polydimethylsiloxane films. J. Appl. Phys. 109(5), 054905 (2011)

18. A.I. Egunov, J.G. Korvink, V.A. Luchnikov, Polydimethylsiloxane bilayer films with an embedded spontaneous curvature. Soft Matter. 12(1), 45-52 (2016)

19. Y. Tanaka, K. Sato, T. Shimizu, M. Yamato, T. Okano, T. Kitamori, Biological cells on microchips: new technologies and applications. Biosens. Bioelectron. 23(4), 449-458 (2007)

20. M. Pilarek, P. Neubauer, U. Marx, Biological cardio-micropumps for microbioreactors and analytical micro-systems. Sens. Actuators B: Chem. 156(2), 517-526 (2011)

21. Y. Tanaka, H. Fujita, Fluid driving system for a micropump by differentiating iPS cells into cardiomyocytes on a tent-like structure. Sens. Actuators B: Chem. 210, 267-272 (2015)

22. R.W. Carlsen, M. Sitti, Bio-hybrid cell-based actuators for microsystems. Small 10(19), 3831-3851 (2014)

23. L. Vannozzi, L. Ricotti, M. Cianchetti, C. Bearzi, C. Gargioli, R. Rizzi, P. Dario, A. Menciassi, Self-assembly of polydimethylsiloxane structures from $2 \mathrm{D}$ to $3 \mathrm{D}$ for bio-hybrid actuation. Bioinspiration Biomim 10(5), 056001 (2015)

24. Z. Gao, H. Li, X. Chen, H.P. Zhang, Using confined bacteria as building blocks to generate fluid flow. Lab Chip 15(24), 4555-4562 (2015) 
25. S.V. Anand, T.A. Saif, Emergent dynamics of cardiomyocyte clusters on deformable polymeric substrates. Extreme Mech. Lett. (2015). doi:10.1016/j.eml.2015.09.008

26. B.L. Gray, A review of magnetic composite polymers applied to microfluidic devices. J. Electrochem. Soc. 161(2), B3173B3183 (2014)

27. J. Li, M. Zhang, L. Wang, W. Li, P. Sheng, W. Wen, Design and fabrication of microfluidic mixer from carbonyl iron-PDMS composite membrane. Microfluidics and nanofluidics 10(4), 919-925 (2011)

28. J.J. Nagel, G. Mikhail, H. Noh, J. Koo, Magnetically actuated micropumps using an Fe-PDMS composite membrane, in Smart Structures and Materials (International Society for Optics and Photonics, 2006), pp. 617213-617213

29. W. Hilber, B. Jakoby, A magnetic membrane actuator in composite technology utilizing diamagnetic levitation. IEEE Sens. J. 13(7), 2786-2791 (2013)

30. W. Hilber, B. Jakoby, Controlled liquid flow in a microfluidic network with pressure sensitive valves based on polydimethylsiloxane (PDMS)/neodymium ( $\mathrm{NdFeB}$ ) composites. Proc. Eng. 47, 382-385 (2012)

31. S. Marchi, A. Casu, F. Bertora, A. Athanassiou, D. Fragouli, Highly magneto-responsive elastomeric films created by a twostep fabrication process. ACS Appl. Mater. Interfaces 7(34), 19112-19118 (2015)

32. J.M. den Toonder, P.R. Onck, Microfluidic manipulation with artificial/bioinspired cilia. Trends Biotechnol. 31(2), 85-91 (2013)

33. J.V. Timonen, C. Johans, K. Kontturi, A. Walther, O. Ikkala, R.H. Ras, A facile template-free approach to magnetodriven, multifunctional artificial cilia. ACS Appl. Mater. Interfaces 2(8), 2226-2230 (2010)

34. Y. Wang, Y. Gao, H.M. Wyss, P.D. Anderson, J.M. den Toonder, Artificial cilia fabricated using magnetic fiber drawing generate substantial fluid flow. Microfluid. Nanofluid. 18(2), 167-174 (2015)

35. M. Riahi, E. Alizadeh, Fabrication of a 3D active mixer based on deformable Fe-doped PDMS cones with magnetic actuation. J. Micromech. Microeng. 22(11), 115001 (2012)

36. M. Rahbar, L. Shannon, B.L. Gray, Microfluidic active mixers employing ultra-high aspect-ratio rare-earth magnetic nanocomposite polymer artificial cilia. J. Micromech. Microeng. 24(2), 025003 (2014)

37. B. Zhou, W. Xu, A.A. Syed, Y. Chau, L. Chen, B. Chew, W. Wen, Design and fabrication of magnetically functionalized flexible micropillar arrays for rapid and controllable microfluidic mixing. Lab Chip 15(9), 2125-2132 (2015)

38. B.L. Fiser, A.R. Shields, M.R. Falvo, R. Superfine, Highly responsive core-shell microactuator arrays for use in viscous and viscoelastic fluids. J. Micromech. Microeng. 25(2), 025004 (2015)

39. F. Fahrni, M.W. Prins, L.J. van Ijzendoorn, Micro-fluidic actuation using magnetic artificial cilia. Lab Chip 9(23), 3413-3421 (2009)

40. F. Liu, G. Alici, B. Zhang, S. Beirne, W. Li, Fabrication and characterization of a magnetic micro-actuator based on deformable Fe-doped PDMS artificial cilium using 3D printing. Smart Mater. Struct. 24(3), 035015 (2015)

41. O.O. Osman, H. Shintaku, S. Kawano, Development of microvibrating flow pumps using MEMS technologies. Microfluid. Nanofluid. 13(5), 703-713 (2012)

42. W. Hilber, S. Clara, B. Jakoby, Magnetic circuits based on polymer composites for controlled actuation of a ferromagnetic sphere at a microfluidic junction. In Proceedings of Sensors, (IEEE Sensors, Baltimore, 2013) pp. 1-4
43. S. Peng, M. Zhang, X. Niu, W. Wen, P. Sheng, Z. Liu, J. Shi, Magnetically responsive elastic microspheres. Appl. Phys. Lett. 92(1), 012108 (2008)

44. D. Liu, K. Bastiaansen, D. Broer, P. Onck, J. den Toonder, Inkjet printing of magnetic/non-magnetic polymer microfluidic actuators, in Proceedings of 14th MicroTAS, 2010, pp. 515-517

45. B. Samel, P. Griss, G. Stemme, A thermally responsive PDMS composite and its microfluidic applications. J. Microelectromech. Syst. 16(1), 50-57 (2007)

46. B. Samel, J. Chretien, R. Yue, P. Griss, G. Stemme, Wafer-level process for single-use buckling-film microliter-range pumps. J. Microelectromech. Syst. 16(4), 795-801 (2007)

47. L. Metref, F. Bianchi, V. Vallet, N. Blanc, R. Goetschmann, P. Renaud, Microfluidic system based on thermoexpandable polymer for on chip blood coagulation testing. Micro Nanosyst. 1(1), 41-45 (2009)

48. G.P. Kanakaris, N. Fatsis-Kavalopoulos, L.G. Alexopoulos, Laser activated single-use micropumps. Sens. Actuators B: Chem. 220, 549-556 (2015)

49. J. Loomis, P. Xu, B. Panchapakesan, Stimuli-responsive transformation in carbon nanotube/expanding microsphere-polymer composites. Nanotechnology 24(18), 185703 (2013)

50. L.Z. Chen, C.H. Liu, C.H. Hu, S.S. Fan, Electrothermal actuation based on carbon nanotube network in silicone elastomer. Appl. Phys. Lett. 92(26), 263104 (2008)

51. S. Cheng, Z. Wu, Microfluidic electronics. Lab Chip 12(16), 2782-2791 (2012)

52. A.P. Gerratt, B. Balakrisnan, I. Penskiy, S. Bergbreiter, Dielectric elastomer actuators fabricated using a micro-molding process. Smart Mater. Struct. 23(5), 055004 (2014)

53. X.Z. Niu, S.L. Peng, L.Y. Liu, W.J. Wen, P. Sheng, Characterizing and patterning of PDMS-based conducting composites. Adv. Mater. 19(18), 2682 (2007)

54. F. Borghi, C. Melis, C. Ghisleri, A. Podestà, L. Ravagnan, L. Colombo, P. Milani, Stretchable nanocomposite electrodes with tunable mechanical properties by supersonic cluster beam implantation in elastomers. Appl. Phys. Lett. 106(12), 121902 (2015)

55. J.D. Tice, T.A. Bassett, A.V. Desai, C.A. Apblett, P.J. Kenis, A monolithic poly (dimethylsiloxane) electrostatic actuator for controlling integrated pneumatic microsystems. Sens. Actuators A: Phys. 196, 22-29 (2013)

56. L. Qu, Q. Peng, L. Dai, G.M. Spinks, G.G. Wallace, R.H. Baughman, Carbon nanotube electroactive polymer materials: opportunities and challenges. MRS Bull. 33(03), 215-224 (2008)

57. U. Kosidlo, M. Omastová, M. Micusík, G. Ćirić-Marjanović, H. Randriamahazaka, T. Wallmersperger, A. Aabloo, I. Kolaric, T. Bauernhansl, Nanocarbon based ionic actuators-a review. Smart Mater. Struct. 22(10), 104022 (2013)

58. L. Wang, M. Zhang, J. Li, X. Gong, W. Wen, Logic control of microfluidics with smart colloid. Lab Chip 10(21), 2869-2874 (2010)

59. C. Goll, W. Bacher, B. Büstgens, D. Maas, W. Menz, W.K. Schomburg, Microvalves with bistable buckled polymer diaphragms. J. Micromech. Microeng. 6(1), 77 (1996)

60. W.K. Schomburg, C. Goll, Design optimization of bistable microdiaphragm valves. Sens. Actuators A: Phys. 64(3), 259-264 (1998)

61. G.E. Song, K.H. Kim, Y.P. Lee, Simulation and experiments for a phase-change actuator with bistable membrane. Sens. Actuators A: Phys. 136(2), 665-672 (2007)

62. B. Yang, B. Wang, W.K. Schomburg, A thermopneumatically actuated bistable microvalve. J. Micromech. Microeng. 20(9), 095024 (2010) 
63. A. Chen, T. Pan, Manually operatable on-chip bistable pneumatic microstructures for microfluidic manipulations. Lab Chip 14(17), 3401-3408 (2014)

64. R. Pelrine, R. Kornbluh, Q. Pei, J. Joseph, High-speed electrically actuated elastomers with strain greater than $100 \%$. Science 287, 836-839 (2000)

65. N. Goulbourne, E. Mockensturm, M. Frecker, A nonlinear model for dielectric elastomer membranes. J. Appl. Mech. 72(6), 899-906 (2005)

66. J.J. Loverich, I. Kanno, H. Kotera, Concepts for a new class of all-polymer micropumps. Lab Chip 6(9), 1147-1154 (2006)

67. C. Murray, D. McCoul, E. Sollier, T. Ruggiero, X. Niu, Q. Pei, D. Di Carlo, Electro-adaptive microfluidics for active tuning of channel geometry using polymer actuators. Microfluid. Nanofluid. 14(1-2), 345-358 (2013)

68. Y. Tanaka, T. Fujikawa, Y. Kazoe, T. Kitamori, An active valve incorporated into a microchip using a high strain electroactive polymer. Sens. Actuators B: Chem. 184, 163-169 (2013)

69. F. Carpi, G. Frediani, S. Turco, D. De Rossi, Bioinspired tunable lens with muscle-like electroactive elastomers. Adv. Funct. Mater. 21(21), 4152-4158 (2011)

70. S. Shian, R.M. Diebold, D.R. Clarke, Tunable lenses using transparent dielectric elastomer actuators. Opt. Express 21(7), 8669-8676 (2013)

71. L. Maffli, S. Rosset, M. Ghilardi, F. Carpi, H. Shea, Ultrafast All-Polymer Electrically Tunable Silicone Lenses. Adv. Funct. Mater. 25(11), 1656-1665 (2015)

72. A. Lamberti, M. Di Donato, A. Chiappone, F. Giorgis, G. Canavese, Tunable electromechanical actuation in silicone dielectric film. Smart Mater. Struct 23(10), 105001 (2014)

73. T. Hoffstadt, J. Maas, Analytical modeling and optimization of DEAP-based multilayer stack-transducers. Smart Mater. Struct. 24(9), 094001 (2015)

74. M. Follador, A.T. Conn, J. Rossiter, Bistable minimum energy structures (BiMES) for binary robotics. Smart Mater. Struct. 24(6), 065037 (2015)

75. L. Zhang, D. Wang, P. Hu, J.W. Zha, F. You, S.T. Li, Z.M. Dang, Highly improved electro-actuation of dielectric elastomers by molecular grafting of azobenzenes to silicon rubber. J. Mater. Chem. C 3(19), 4883-4889 (2015)

76. M. Kollosche, G. Kofod, Z. Suo, J. Zhu, Temporal evolution and instability in a viscoelastic dielectric elastomer. J. Mech. Phys. Solids 76, 47-64 (2015)

77. N. Pekas, Q. Zhang, M. Nannini, D. Juncker, Wet-etching of structures with straight facets and adjustable taper into glass substrates. Lab Chip 10(4), 494-498 (2010)

78. M.P. Chang, M.M. Maharbiz, Electrostatically-driven elastomer components for user-reconfigurable high density microfluidics. Lab Chip 9(9), 1274-1281 (2009)

79. J.D. Tice, A.V. Desai, T.A. Bassett, C.A. Apblett, P.J. Kenis, Control of pressure-driven components in integrated microfluidic devices using an on-chip electrostatic microvalve. RSC Adv. 4(93), 51593-51602 (2014)

80. A.V. Desai, J.D. Tice, C.A. Apblett, P.J. Kenis, Design considerations for electrostatic microvalves with applications in poly (dimethylsiloxane)-based microfluidics. Lab Chip 12(6), 1078-1088 (2012)

81. E. Yıldırım, M.S. Arıkan, H. Külah, A normally closed electrostatic parylene microvalve for micro total analysis systems. Sens. Actuators A: Phys. 181, 81-86 (2012)

82. N. Pekas, Q. Zhang, D. Juncker, Electrostatic actuator with liquid metal-elastomer compliant electrodes used for on-chip microvalving. J. Micromech. Microeng. 22(9), 097001 (2012)

83. S. Wagner, S. Bauer, Materials for stretchable electronics. MRS Bull. 37(03), 207-213 (2012)
84. J.H. Kim, K.T. Lau, R. Shepherd, Y. Wu, G. Wallace, D. Diamond, Performance characteristics of a polypyrrole modified polydimethylsiloxane (PDMS) membrane based microfluidic pump. Sens. Actuators A: Phys. 148(1), 239-244 (2008)

85. J.J. Wang, T.H. Hsu, C.N. Yeh, J.W. Tsai, Y.C. Su, Piezoelectric polydimethylsiloxane films for MEMS transducers. J. Micromech. Microeng. 22(1), 015013 (2012)

86. J.W. Tsai, J.J. Wang, Y.C. Su, Piezoelectric rubber films for autonomous physiological monitoring systems. Sens. Actuators A: Phys. 215, 176-183 (2014)

87. W. Hilber, S. Clara, B. Jakoby, Microfluidic pumping utilizing a PDMS membrane with an integrated nonuniform open-porous foam. IEEE Sens. J. 15(9), 5109-5114 (2015)

88. P.H. Huang, N. Nama, Z. Mao, P. Li, J. Rufo, Y. Chen, Y. Xie, C.H. Wei, L. Wang, T.J. Huang, A reliable and programmable acoustofluidic pump powered by oscillating sharp-edge structures. Lab Chip 14(22), 4319-4323 (2014)

89. K. Oh, J.H. Chung, S. Devasia, J.J. Riley, Bio-mimetic silicone cilia for microfluidic manipulation. Lab chip 9(11), 1561-1566 (2009)

90. K. Oh, B. Smith, S. Devasia, J.J. Riley, J.H. Chung, Characterization of mixing performance for bio-mimetic silicone cilia. Microfluid. Nanofluid. 9(4-5), 645-655 (2010)

91. T.R. Lee, K. Oh, J.H. Chung, Y.S. Chang, J.B. Choi, G. Yagawa, Y.J. Kim, Resonant behavior and microfluidic manipulation of silicone cilia due to an added mass effect. Soft Matter. 7(9), 4325-4334 (2011)

92. H.B. Lee, K. Oh, W.H. Yeo, T.R. Lee, Y.S. Chang, J.B. Choi, K.H. Lee, J.H. Chung, Enhanced bioreaction efficiency of a microfluidic mixer toward high-throughput and low-cost bioassays. Microfluid. Nanofluid. 12(1-4), 143-156 (2012)

93. A. Rockenbach, V. Mikulich, C. Brücker, U. Schnakenberg, Fluid transport via pneumatically actuated waves on a ciliated wall. J. Micromech. Microeng. 25(12), 125009 (2015)

94. B. Gorissen, M. de Volder, D. Reynaerts, Pneumatically-actuated artificial cilia array for biomimetic fluid propulsion. Lab Chip 15(22), 4348-4355 (2015)

95. D. Snakenborg, H. Klank, J.P. Kutter, Polymer microvalve with pre-stressed membranes for tunable flow-pressure characteristics. Microfluid. Nanofluid. 10(2), 381-388 (2011)

96. Y.N. Wang, C.H. Tsai, L.M. Fu, L.K.L. Liou, Microfluidic rectifier based on poly (dimethylsiloxane) membrane and its application to a micropump. Biomicrofluidics 7(4), 044118 (2013)

97. R. Mohan, B.R. Schudel, A.V. Desai, J.D. Yearsley, C.A. Apblett, P.J. Kenis, Design considerations for elastomeric normally closed microfluidic valves. Sens. Actuators B: Chem. 160(1), 1216-1223 (2011)

98. T. Mazzocchi, L. Ricotti, N. Pinzi, A. Menciassi, Parametric design, fabrication and validation of one-way polymeric valves for artificial sphincters. Sens. Actuators A: Phys. 233, 184-194 (2015)

99. M.E. Piyasena, R. Newby, T.J. Miller, B. Shapiro, E. Smela, Electroosmotically driven microfluidic actuators. Sens. Actuators B: Chem. 141(1), 263-269 (2009)

100. D. Sritharan, A.S. Chen, P. Aluthgama, B. Naved, E. Smela, Bubble-free electrokinetic flow with propylene carbonate. Electrophoresis 36(20), 2622-2629 (2015)

101. D.J. Beebe, J.S. Moore, J.M. Bauer, Q. Yu, R.H. Liu, C. Devadoss, B.H. Jo, Functional hydrogel structures for autonomous flow control inside microfluidic channels. Nature 404(6778), 588-590 (2000)

102. L. Ionov, Hydrogel-based actuators: possibilities and limitations. Mater. Today 17(10), 494-503 (2014) 
103. Z. Liu, W. Toh, T.Y. Ng, Advances in mechanics of soft materials: a review of large deformation behavior of hydrogels. Int. J. Appl. Mech. 7(05), 1530001 (2015)

104. L. Dong, H. Jiang, Autonomous microfluidics with stimuli-responsive hydrogels. Soft Matter. 3(10), 1223-1230 (2007)

105. S.M. Kim, B. Lee, H. Yoon, K.Y. Suh, Stimuli-responsive hydrogel patterns for smart microfluidics and microarrays. Analyst 138(21), 6230-6242 (2013)

106. B.D. Kieviet, P.M. Schön, G.J. Vancso, Stimulus-responsive polymers and other functional polymer surfaces as components in glass microfluidic channels. Lab Chip 14(21), 4159-4170 (2014)

107. R.H. Liu, Q. Yu, D.J. Beebe, Fabrication and characterization of hydrogel-based microvalves. J. Microelectromech. Syst. 11(1), 45-53 (2002)

108. J.Y. Park, H.J. Oh, D.J. Kim, J.Y. Baek, S.H. Lee, A polymeric microfluidic valve employing a $\mathrm{pH}$-responsive hydrogel microsphere as an actuating source. J. Micromech. Microeng. 16(3), 656 (2006)

109. D. Kim, S. Kim, J. Park, J. Baek, S. Kim, K. Sun, T. Lee, S. Lee, Hydrodynamic fabrication and characterization of a $\mathrm{pH}$-responsive microscale spherical actuating element. Sens. Actuators A: Phys. 134(2), 321-328 (2007)

110. A. Atwe, A. Gupta, R. Kant, M. Das, I. Sharma, S. Bhattacharya, A novel microfluidic switch for $\mathrm{pH}$ control using Chitosan based hydrogels. Microsyst. Technol. 20(7), 1373-1381 (2014)

111. L.D. Zarzar, P. Kim, J. Aizenberg, Bio-inspired design of submerged hydrogel-actuated polymer microstructures operating in response to $\mathrm{pH}$. Adv. Mater. 23(12), 1442-1446 (2011)

112. V. Gopishetty, Y. Roiter, I. Tokarev, S. Minko, Multiresponsive biopolyelectrolyte membrane. Adv. Mater. 20(23), 4588-4593 (2008)

113. Q. Yang, N. Adrus, F. Tomicki, M. Ulbricht, Composites of functional polymeric hydrogels and porous membranes. J. Mater. Chem. 21(9), 2783-2811 (2011)

114. P. Techawanitchai, M. Ebara, N. Idota, T.A. Asoh, A. Kikuchi, T. Aoyagi, Photo-switchable control of $\mathrm{pH}$-responsive actuators via $\mathrm{pH}$ jump reaction. Soft Matter. 8(10), 2844-2851 (2012)

115. J.C. Kurnia, E. Birgersson, A.S. Mujumdar, Computational study of $\mathrm{pH}$-sensitive hydrogel-based microfluidic flow controllers. J. Funct. Biomater. 2(3), 195-212 (2011)

116. Y. Zhang, Z. Liu, S. Swaddiwudhipong, H. Miao, Z. Ding, Z. Yang, pH-Sensitive hydrogel for micro-fluidic valve. J. Funct. Biomater. 3(3), 464-479 (2012)

117. S. Sugiura, K. Sumaru, K. Ohi, K. Hiroki, T. Takagi, T. Kanamori, Photoresponsive polymer gel microvalves controlled by local light irradiation. Sens. Actuators A: Phys. 140(2), 176-184 (2007)

118. G. Chen, F. Svec, D.R. Knapp, Light-actuated high pressureresisting microvalve for on-chip flow control based on thermoresponsive nanostructured polymer. Lab Chip 8(7), 1198-1204 (2008)

119. E. Wang, M.S. Desai, S.W. Lee, Light-controlled grapheneelastin composite hydrogel actuators. Nano Lett. 13(6), 2826-2830 (2013)

120. W. Toh, T.Y. Ng, J. Hu, Z. Liu, Mechanics of inhomogeneous large deformation of photo-thermal sensitive hydrogels. Int. J. Solids Struct. 51(25), 4440-4451 (2014)

121. A.D. Jadhav, B. Yan, R.C. Luo, L. Wei, X. Zhen, C.H. Chen, P. Shi, Photoresponsive microvalve for remote actuation and flow control in microfluidic devices. Biomicrofluidics 9(3), 034114 (2015)

122. C. Yu, S. Mutlu, P. Selvaganapathy, C.H. Mastrangelo, F. Svec, J.M. Fréchet, Flow control valves for analytical microfluidic chips without mechanical parts based on thermally responsive monolithic polymers. Analyt. Chem. 75(8), 1958-1961 (2003)

123. A. Richter, D. Kuckling, S. Howitz, T. Gehring, K.F. Arndt, Electronically controllable microvalves based on smart hydrogels: magnitudes and potential applications. J. Microelectromech. Syst. 12(5), 748-753 (2003)

124. J. Wang, Z. Chen, M. Mauk, K.S. Hong, M. Li, S. Yang, H.H. Bau, Self-actuated, thermo-responsive hydrogel valves for lab on a chip. Biomed. Microdev. 7(4), 313-322 (2005)

125. M. Bäcker, M. Raue, S. Schusser, C. Jeitner, L. Breuer, P. Wagner, A. Poghossian, T. Mang, M.J. Schöning, Microfluidic chip with integrated microvalves based on temperature-and $\mathrm{pH}-$ responsive hydrogel thin films. Phys. Status solidi (a) 209(5), 839-845 (2012)

126. M.E. Harmon, M. Tang, C.W. Frank, A microfluidic actuator based on thermoresponsive hydrogels. Polymer 44(16), 4547-4556 (2003)

127. A. Li, A. Khosla, C. Drewbrook, B.L. Gray, Fabrication and testing of thermally responsive hydrogel-based actuators using polymer heater elements for flexible microvalves, In SPIE MOEMS-MEMS, 79290G-79290G (2011)

128. H. Mazaheri, M. Baghani, R. Naghdabadi, S. Sohrabpour, Inhomogeneous swelling behavior of temperature sensitive PNIPAM hydrogels in micro-valves: analytical and numerical study. Smart Mater. Struct. 24(4), 045004 (2015)

129. A. Richter, S. Klatt, G. Paschew, C. Klenke, Micropumps operated by swelling and shrinking of temperature-sensitive hydrogels. Lab Chip 9(4), 613-618 (2009)

130. E.J. Geiger, A.P. Pisano, F. Svec, A polymer-based microfluidic platform featuring on-chip actuated hydrogel valves for disposable applications. J. Microelectromech. Syst. 19(4), 944-950 (2010)

131. A.K. Agarwal, S.S. Sridharamurthy, D.J. Beebe, H. Jiang, Programmable autonomous micromixers and micropumps. J. Microelectromech. Syst. 14(6), 1409-1421 (2005)

132. S. Lin, W. Wang, X.J. Ju, R. Xie, L.Y. Chu, A simple strategy for in situ fabrication of a smart hydrogel microvalve within microchannels for thermostatic control. Lab Chip 14(15), 2626-2634 (2014)

133. X. Huang, Y. Sun, S. Soh, Stimuli-responsive surfaces for tunable and reversible control of wettability. Adv. Mater. 27(27), 4062-4068 (2015)

134. F. Benito-Lopez, M. Antoñana-Díez, V.F. Curto, D. Diamond, V. Castro-López, Modular microfluidic valve structures based on reversible thermoresponsive ionogel actuators. Lab Chip 14(18), 3530-3538 (2014)

135. V. Stroganov, M. Al-Hussein, J.U. Sommer, A. Janke, S. Zakharchenko, L. Ionov, Reversible thermosensitive biodegradable polymeric actuators based on confined crystallization. Nano Lett. 15(3), 1786-1790 (2015)

136. S.E. Bakarich, R. Gorkin, G.M. Spinks, 4D printing with mechanically robust, thermally actuating hydrogels. Macromol Rapid Commun. 36(12), 1211-1217 (2015)

137. N.S. Satarkar, W. Zhang, R.E. Eitel, J.Z. Hilt, Magnetic hydrogel nanocomposites as remote controlled microfluidic valves. Lab Chip 9(12), 1773-1779 (2009)

138. P. Ilg, Stimuli-responsive hydrogels cross-linked by magnetic nanoparticles. Soft Matter. 9(13), 3465-3468 (2013)

139. L. Roeder, P. Bender, M. Kundt, A. Tschöpe, A.M. Schmidt, Magnetic and geometric anisotropy in particle-crosslinked ferrohydrogels. Phys. Chem. Chem. Phys. 17(2), 1290-1298 (2015)

140. G.H. Kwon, Y.Y. Choi, J.Y. Park, D.H. Woo, K.B. Lee, J.H. Kim, S.H. Lee, Electrically-driven hydrogel actuators in microfluidic channels: fabrication, characterization, and biological application. Lab Chip 10(12), 1604-1610 (2010) 
141. G.H. Kwon, G.S. Jeong, J.Y. Park, J.H. Moon, S.H. Lee, A lowenergy-consumption electroactive valveless hydrogel micropump for long-term biomedical applications. Lab Chip 11(17), 2910-2915 (2011)

142. J.R.C. Saunders, W. Moussa, Cyclical electrical stimulation of hydrogel microactuators employing parylene-N coated electrodes. J. Microelectromech. Syst. 23(1), 230-242 (2014)

143. J. Xue, C. Hu, L. Lv, L. Dai, L. Qu, Re-shaping graphene hydrogels for effectively enhancing actuation responses. Nanoscale 7(29), 12372-12378 (2015)

144. J. Nestler, A. Morschhauser, K. Hiller, T. Otto, S. Bigot, J. Auerswald, H.F. Knapp, J. Gavillet, T. Gessner, Polymer lab-onchip systems with integrated electrochemical pumps suitable for large-scale fabrication. Int. J. Adv. Manuf. Technol. 47(1-4), 137-145 (2010)

145. B.T. Good, C.N. Bowman, R.H. Davis, Modeling and verification of fluid-responsive polymer pumps for microfluidic systems. Chem. Eng. Sci. 59(24), 5967-5974 (2004)

146. B.T. Good, C.N. Bowman, R.H. Davis, A water-activated pump for portable microfluidic applications. J. Colloid Interface Sci. 305(2), 239-249 (2007)

147. M. Vosseler, M. Clemenz, R. Zengerle, A flat and cost effective actuator based on superabsorbent polymer driving a skin attachable drug delivery system. Smart Mater. Struct. 21(10), 105002 (2012)

148. Y.H. Choi, K.H. Chung, S.S. Lee, Microfluidic actuation by dehydration of hydrogel, in Proceedings of Sensors, (IEEE Sensors, Christchurch, 2009) pp. 1370-1373

149. H. Kuroki, C. Islam, I. Tokarev, H. Hu, G. Liu, S. Minko, Tunable ultrathin membranes with non-volatile pore shape memory. ACS Appl. Mater. Interfaces 7(19), 10401-10406 (2015)

150. Y. Murase, S. Maeda, S. Hashimoto, R. Yoshida, Design of a mass transport surface utilizing peristaltic motion of a selfoscillating gel. Langmuir 25(1), 483-489 (2008)

151. S. Maeda, Y. Hara, R. Yoshida, S. Hashimoto, Control of the dynamic motion of a gel actuator driven by the BelousovZhabotinsky reaction. Macromol. Rapid Commun. 29(5), 401-405 (2008)

152. Y. Hara, R. Yoshida, Self-oscillating polymer fueled by organic acid. J. Phys. Chem. B 112(29), 8427-8429 (2008)

153. Y. Zhang, N. Zhou, N. Li, M. Sun, D. Kim, S. Fraden, I.R. Epstein, B. Xu, Giant volume change of active gels under continuous flow. J. Am. Chem. Soc. 136(20), 7341-7347 (2014)

154. H. Zhou, Z. Zheng, Q. Wang, G. Xu, J. Li, X. Ding, A modular approach to self-oscillating polymer systems driven by the Belousov-Zhabotinsky reaction. RSC Adv. 5(18), 13555-13569 (2015)

155. H. Jiang, C. Li, X. Huang, Actuators based on liquid crystalline elastomer materials. Nanoscale 5(12), 5225-5240 (2013)

156. Y. Ji, J.E. Marshall, E.M. Terentjev, Nanoparticle-liquid crystalline elastomer composites. Polymers 4(1), 316-340 (2012)

157. T.J. White, D.J. Broer, Programmable and adaptive mechanics with liquid crystal polymer networks and elastomers. Nat. Mater. 14(11), 1087-1098 (2015)

158. C.L. van Oosten, C.W. Bastiaansen, D.J. Broer, Printed artificial cilia from liquid-crystal network actuators modularly driven by light. Nat. Mater. 8(8), 677-682 (2009)

159. M. Chen, X. Xing, Z. Liu, Y. Zhu, H. Liu, Y. Yu, F. Cheng, Photodeformable polymer material: towards light-driven micropump applications. Appl. Phys. A 100(1), 39-43 (2010)

160. Y. Yang, W. Zhan, R. Peng, C. He, X. Pang, D. Shi, T. Jiang, Z. Lin, Graphene-enabled superior and tunable photomechanical actuation in liquid crystalline elastomer nanocomposites. Adv. Mater. 27(41), 6376-6381 (2015)
161. A. Sánchez-Ferrer, T. Fischl, M. Stubenrauch, A. Albrecht, H. Wurmus, M. Hoffmann, H. Finkelmann, Liquid-crystalline elastomer microvalve for microfluidics. Adv. Mater. 23(39), 4526-4530 (2011)

162. E.K. Fleischmann, H.L. Liang, N. Kapernaum, F. Giesselmann, J. Lagerwall, R. Zentel, One-piece micropumps from liquid crystalline core-shell particles. Nat. Commun. 3, 1178 (2012)

163. F. Greco, V. Domenici, A. Desii, E. Sinibaldi, B. Zupančič, B. Zalar, B. Mazzolai, V. Mattoli, Liquid single crystal elastomer/conducting polymer bilayer composite actuator: modelling and experiments. Soft Matter. 9(47), 11405-11416 (2013)

164. H. Ren, S. Xu, S.T. Wu, Liquid crystal pump. Lab Chip 13(1), 100-105 (2013)

165. S. Bauer, R. Gerhard-Multhaupt, G.M. Sessler, Ferroelectrets: soft electroactive foams for transducers. Phys. Today 57(2), 37-43 (2004)

166. K.S. Ramadan, D. Sameoto, S. Evoy, A review of piezoelectric polymers as functional materials for electromechanical transducers. Smart Mater. Struct. 23(3), 033001 (2014)

167. T.B. Xu, J. Su, Development, characterization, and theoretical evaluation of electroactive polymer-based micropump diaphragm. Sens. Actuators A: Phys. 121(1), 267-274 (2005)

168. F. Xia, S. Tadigadapa, Q.M. Zhang, Electroactive polymer based microfluidic pump. Sens. Actuators A: Phys. 125(2), 346-352 (2006)

169. O. Pabst, J. Perelaer, E. Beckert, U.S. Schubert, R. Eberhardt, A. Tünnermann, All inkjet-printed piezoelectric polymer actuators: characterization and applications for micropumps in lab-on-achip systems. Org. Electron. 14(12), 3423-3429 (2013)

170. O. Pabst, S. Hölzer, E. Beckert, J. Perelaer, U.S. Schubert, R. Eberhardt, A. Tünnermann, Inkjet printed micropump actuator based on piezoelectric polymers: device performance and morphology studies. Org. Electron. 15(11), 3306-3315 (2014)

171. E. Allahyarov, H. Löwen, L. Zhu, Analysis of the actuation properties of charged multilayer films. J. Appl. Phys. 117(3), 034504 (2015)

172. H.H. Wang, T.J. Wu, C.C Hsu, C.K. Lee, Y.H. Hsu, A lightactivated optopiezoelectric thin-film actuator for microfluidic applications. in SPIE BiOS, (International Society for Optics and Photonics, 2015), pp. 93200D-93200D

173. M.Q. Le, J.F. Capsal, J. Galineau, F. Ganet, X. Yin, M.D. Yang, J.F. Chateaux, L. Renaud, C. Malhaire, P.J. Cottinet, R. Liang, All-organic electrostrictive polymer composites with low driving electrical voltages for micro-fluidic pump applications. Nat. Sci. Rep. 5, 11814 (2015)

174. K. Kruusamäe, A. Punning, A. Aabloo, K. Asaka, Self-sensing ionic polymer actuators: a review. Actuators 4(1), 17-38 (2015)

175. V. De Luca, P. Digiamberardino, G. Di Pasquale, S. Graziani, A. Pollicino, E. Umana, M.G. Xibilia, Ionic electroactive polymer metal composites: fabricating, modeling, and applications of postsilicon smart devices. J. Polymer Sci. Part B: Polymer Phys. 51(9), 699-734 (2013)

176. C. Jo, D. Pugal, I.K. Oh, K.J. Kim, K. Asaka, Recent advances in ionic polymer-metal composite actuators and their modeling and applications. Prog. Polymer Sci. 38(7), 1037-1066 (2013)

177. S. Lee, K.J. Kim, Design of IPMC actuator-driven valve-less micropump and its flow rate estimation at low Reynolds numbers. Smart Mater. Struct. 15(4), 1103 (2006)

178. T.T. Nguyen, N.S. Goo, V.K. Nguyen, Y. Yoo, S. Park, Design, fabrication, and experimental characterization of a flap valve IPMC micropump with a flexibly supported diaphragm. Sens. Actuators A: Phys. 141(2), 640-648 (2008)

179. D.N.C. Nam, K.K. Ahn, Design of an IPMC diaphragm for micropump application. Sens. Actuators A: Phys. 187, 174-182 (2012) 
180. C.M. Meis, R. Montazami, N. Hashemi, Ionic electroactive polymer actuators as active microfluidic mixers. Anal. Methods 7(24), 10217-10223 (2015)

181. C.M. Meis, N. Hashemi, R. Montazami, Investigation of spraycoated silver-microparticle electrodes for ionic electroactive polymer actuators. J. Appl. Phys. 115(13), 134302 (2014)

182. V. Panwar, J.H. Jeon, G. Anoop, H.J. Lee, I.K. Oh, J.Y. Jo, Low voltage actuator using ionic polymer metal nanocomposites based on a miscible polymer blend. J. Mater. Chem. A 3(39), 19718-19727 (2015)

183. A. Punning, I. Must, I. Põldsalu, V. Vunder, F. Kaasik, R. Temmer, A. Aabloo, Long-term degradation of the ionic electroactive polymer actuators, in SPIE Smart Structures and Materials + Nondestructive Evaluation and Health Monitoring, (2015), pp. 94300S-94300S

184. J.D. Carrico, N.W. Traeden, M. Aureli, K.K. Leang, Fused filament 3D printing of ionic polymer-metal composites (IPMCs). Smart Mater. Struct. 24(12), 125021 (2015)

185. K. Kruusamäe, K. Mukai, T. Sugino, K. Asaka, Mechanical behaviour of bending bucky-gel actuators and its representation. Smart Mater. Struct. 23(2), 025031 (2014)

186. K. Kruusamäe, K. Mukai, T. Sugino, K. Asaka, Impact of viscoelastic properties on bucky-gel actuator performance. J. Intell. Mater. Syst. Struct. 25(18), 2235-2245 (2014)

187. K. Kruusamäe, T. Sugino, K. Asaka, Ionic and viscoelastic mechanisms of a bucky-gel actuator. J. Appl. Phys. 118(1), $014502(2015)$

188. A.K. Ghamsari, E. Zegeye, Y. Jin, E. Woldesenbet, Application of bucky gel in fabrication of a low-voltage rapid microvalve for flow regulation. ACS Appl. Mater. Interfaces 5(12), 5408-5412 (2013)

189. E.W.H. Jager, E. Smela, O. Inganäs, Microfabricating conjugated polymer actuators. Science 290(5496), 1540-1545 (2000)

190. Y. Wu, D. Zhou, G.M. Spinks, P.C. Innis, W.M. Megill, G.G. Wallace, TITAN: a conducting polymer based microfluidic pump. Smart Mater. Struct. 14(6), 1511 (2005)

191. M. Fuchiwaki, Y. Naka, K. Tanaka, Performance of a micro pump driven by conducting polymer soft actuator based on polypyrrole. Adv. Mater. Res. 93, 615-618 (2010)

192. Y. Fang, X. Tan, A novel diaphragm micropump actuated by conjugated polymer petals: fabrication, modeling, and experimental results. Sens. Actuators A: Phys. 158(1), 121-131 (2010)

193. S. Taccola, F. Greco, E. Sinibaldi, A. Mondini, B. Mazzolai, V. Mattoli, Toward a new generation of electrically controllable hygromorphic soft actuators. Adv. Mater. 27(10), 1668-1675 (2015)

194. R. Pal, M. Yang, B.N. Johnson, D.T. Burke, M.A. Burns, Phase change microvalve for integrated devices. Anal. Chem. 76(13), 3740-3748 (2004)

195. S. Ogden, R. Bodén, K. Hjort, A latchable valve for highpressure microfluidics. J. Microelectromech. Syst. 19(2), 396-401 (2010)

196. S. Ogden, L. Klintberg, G. Thornell, K. Hjort, R. Bodén, Review on miniaturized paraffin phase change actuators, valves, and pumps. Microfluid. Nanofluid. 17(1), 53-71 (2014)

197. K. Pitchaimani, B.C. Sapp, A. Winter, A. Gispanski, T. Nishida, Z.H. Fan, Manufacturable plastic microfluidic valves using thermal actuation. Lab Chip 9(21), 3082-3087 (2009)

198. S. Augustine, P. Gu, X. Zheng, T. Nishida, Z.H. Fan, Lowpower electrically controlled thermoelastic microvalves integrated in thermoplastic microfluidic devices. Microfluid. Nanofluid. 19(6), 1385-1394 (2015)

199. C. Neumann, A. Voigt, L. Pires, B.E. Rapp, Design and characterization of a platform for thermal actuation of up to 588 microfluidic valves. Microfluid. Nanofluid. 14(1-2), 177-186 (2013)
200. A. Boustheen, F.G.A. Homburg, M.G.A.M. Somhorst, A. Dietzel, A layered modular polymeric $\mu$-valve suitable for lab-onfoil: design, fabrication, and characterization. Microfluid. Nanofluid. 11(6), 663-673 (2011)

201. D. Copic, A.J. Hart, Corrugated paraffin nanocomposite films as large stroke thermal actuators and self-activating thermal interfaces. ACS Appl. Mater. Interfaces 7(15), 8218-8224 (2015)

202. Y.J. Jin, B.S.I. Kim, W.E. Lee, C.L. Lee, H. Kim, K.H. Song, S.Y. Jang, G. Kwak, Phase-change hybrids for thermo-responsive sensohermos actuators. NPG Asia Mater. 6(10), e137 (2014)

203. L. Sun, W.M. Huang, Z. Ding, Y. Zhao, C.C. Wang, H. Purnawali, C. Tang, Stimulus-responsive shape memory materials: a review. Mater. Des. 33, 577-640 (2012)

204. M. Ebara, K. Uto, N. Idota, J.M. Hoffman, T. Aoyagi, Rewritable and shape-memory soft matter with dynamically tunable microchannel geometry in a biological temperature range. Soft Matter. 9(11), 3074-3080 (2013)

205. A.M. Waddell, J. Punch, J. Stafford, N. Jeffers, On the hydrodynamic characterization of a passive shape memory alloy valve. Appl. Thermal Eng. 75, 731-737 (2015)

206. S. Vyawahare, S. Sitaula, S. Martin, D. Adalian, A. Scherer, Electronic control of elastomeric microfluidic circuits with shape memory actuators. Lab Chip 8(9), 1530-1535 (2008)

207. M.E. Piccini, B.C. Towe, A shape memory alloy microvalve with flow sensing. Sens. Actuators A: Phys. 128(2), 344-349 (2006)

208. M. Zarek, M. Layani, I. Cooperstein, E. Sachyani, D. Cohn, S. Magdassi, 3D Printing of shape memory polymers for flexible electronic devices. Adv. Mater. 28(22), 4449-4454 (2015)

209. L. Zipser, L. Richter, U. Lange, Magnetorheologic fluids for actuators. Sens. Actuators A: Phys. 92(1), 318-325 (2001)

210. H. Hartshorne, C.J. Backhouse, W.E. Lee, Ferrofluid-based microchip pump and valve. Sens. Actuators B: Chem. 99(2), 592-600 (2004)

211. C.Y. Lee, J.C. Leong, Y.N. Wang, L.M. Fu, S.J. Chen, A ferrofluidic magnetic micropump for variable-flow-rate applications. Jpn. J. Appl. Phys. 51(4R), 047201 (2012)

212. K.S. Lok, Y.C. Kwok, P.P.F. Lee, N.T. Nguyen, Ferrofluid plug as valve and actuator for whole-cell PCR on chip. Sens. Actuators B: Chem. 166, 893-897 (2012)

213. M. Ashouri, M.B. Shafii, A. Moosavi, H.A. Hezave, A novel revolving piston minipump. Sens. Actuators B: Chem. 218, 237-244 (2015)

214. R.J. Yang, H.H. Hou, Y.N. Wang, L.M. Fu, Micro-magnetofluidics in microfluidic systems: a review. Sens. Actuators B: Chem. 224, 1-15 (2016)

215. X. Niu, W. Wen, Y.K. Lee, Electrorheological-fluid-based microvalves. Appl. Phys. Lett. 87(24), 243501 (2005)

216. X. Niu, L. Liu, W. Wen, P. Sheng, Active microfluidic mixer chip. Appl. Phys. Lett. 88(15), 153508 (2006)

217. X. Niu, L. Liu, W. Wen, P. Sheng, Hybrid approach to highfrequency microfluidic mixing. Phys. Rev. Letters 97(4), 044501 (2006)

218. L. Liu, X. Chen, X. Niu, W. Wen, P. Sheng, Electrorheological fluid-actuated microfluidic pump. Appl. Phys. Lett. 89(8), 083505 (2006)

219. L. Liu, W. Cao, J. Wu, W. Wen, D.C. Chang, P. Sheng, Design and integration of an all-in-one biomicrofluidic chip. Biomicrofluidics 2(3), 034103 (2008)

220. M. Zhang, L. Wang, X. Wang, J. Wu, J. Li, X. Gong, J. Qin, W. $\mathrm{Li}, \mathrm{W}$. Wen, Microdroplet-based universal logic gates by electrorheological fluid. Soft Matter. 7(16), 7493-7497 (2011)

221. P. Sheng, W. Wen, Electrorheological fluids: mechanisms, dynamics, and microfluidics applications. Ann. Rev. Fluid Mech. 44, 143-174 (2012) 
222. J. Wu, W. Wen, P. Sheng, Smart electroresponsive droplets in microfluidics. Soft Matter. 8(46), 11589-11599 (2012)

223. M.A. Unger, H.P. Chou, T. Thorsen, A. Scherer, S.R. Quake, Monolithic microfabricated valves and pumps by multilayer soft lithography. Science 288(5463), 113-116 (2000)

224. F.B. Madsen, A.E. Daugaard, S. Hvilsted, A.L. Skov, The current state of silicone-based dielectric elastomer transducers. Macromol. Rapid Commun. 37(5), 378-413 (2016)

225. G. Buchberger, B. Hauser, J. Schoeftner, S. Bauer, B. Jakoby, W. Hilber, Temporal change in the electromechanical properties of dielectric elastomer minimum energy structures. J. Appl. Phys. 115(21), 214105 (2014)
226. T.R. Dargaville, M.C. Celina, J.M. Elliott, P.M. Chaplya, G.D. Jones, D.M. Mowery, R.A. Assink, R.L. Clough, J.W. Martin, Characterization, performance and optimization of PVDF as a piezoelectric film for advanced space mirror concepts. Sandia Report SAND2005-6846. Sandia National Laboratories (2005)

227. H. Gong, A.T. Woolley, G.P. Nordin, High density 3D printed microfluidic valves, pumps, and multiplexers. Lab Chip (2016). doi:10.1039/C6LC00565A 